SANDIA REPORT

SAND99-0077

Unlimited Release

Printed January 1999

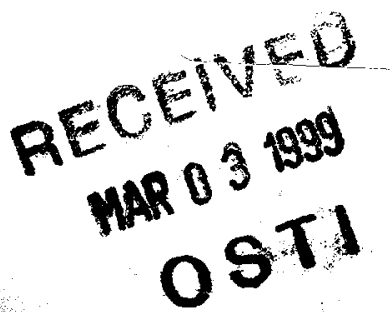

Uniaxial Compression Experiments on PZT 95/5-2 Nb Ceramic: Evidence for an Orientation-Dependent, "Maximum Compressive Stress" Criterion for Onset of the $F_{R 1} \rightarrow A_{0}$ Polymorphic Phase

\title{
Transformation
}

D. H. Zeuch, S. T. Montgomery, D. J. Holcomb, J. M. Grazier, and L. W. Carlson

Prepared by

Sandia National Laboratories

Albuquerque, New Mexico 87, 85 and Livermore, California 94550

Sandia is a multiprogram leboratory operated by Sandia Corporation.

Energy under Contrompany, for the United States Department of

Energy under contract oE AC04-94AL85000.

Approved for public release; further dissemination unlimited.

\section{Sandia National Laboratories}


Issued by Sandia National Laboratories, operated for the United States Department of Energy by Sandia Corporation.

NOTICE: This report was prepared as an account of work sponsored by an agency of the United States Government. Neither the United States Government, nor any agency thereof, nor any of their employees, nor any of their contractors, subcontractors, or their employees, make any warranty, express or implied, or assume any legal liability or responsibility for the accuracy, completeness, or usefulness of any information, apparatus, product, or process disclosed, or represent that its use would not infringe privately owned rights. Reference herein to any specific commercial product, process, or service by trade name, trademark, manufacturer, or otherwise, does not necessarily constitute or imply its endorsement, recommendation, or favoring by the United States Government, any agency thereof, or any of their contractors or subcontractors. The views and opinions expressed herein do not necessarily state or reflect those of the United States Government, any agency thereof, or any of their contractors.

Printed in the United States of America. This report has been reproduced directly from the best available copy.

Available to DOE and DOE contractors from

Office of Scientific and Technical Information

P.O. Box 62

Oak Ridge, TN 37831

Prices available from (703) 605-6000

Web site: http://www.ntis.gov/ordering.htm

Available to the public from

National Technical Information Service

U.S. Department of Commerce

5285 Port Royal Rd

Springfield, VA 22161

NTIS price codes

Printed copy: A04

Microfiche copy: A01

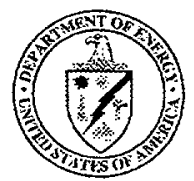




\section{DISCLAIMER}

Portions of this document may be illegible in electronic image products. Images are produced from the best available original document. 


\title{
Uniaxial Compression Experiments on PZT 95/5-2Nb Ceramic: Evidence for an Orientation-Dependent, "Maximum Compressive Stress" Criterion for Onset of the $F_{R 1} \rightarrow A_{O}$ Polymorphic Phase Transformation
}

\author{
D. H. Zeuch \\ Geomechanics Department \\ S. T. Montgomery \\ Integrated Product Development Department \\ D. J. Holcomb, J. M. Grazier and L. W. Carlson \\ Geomechanics Department \\ Sandia National Laboratories \\ P.O. Box 5800 \\ Albuquerque, NM 87185-0751
}

\begin{abstract}
Some time ago we presented evidence that, under nonhydrostatic loading, the $F_{R 1} \rightarrow A_{O}$ polymorphic phase transformation in unpoled PZT $95 / 5-2 \mathrm{Nb}$ ceramic began when the maximum compressive stress equaled the hydrostatic pressure at which the transformation otherwise took place. More recently, we showed that this simple stress criterion did not apply to nonhydrostatically compressed, poled ceramic. However, unpoled ceramic is isotropic, whereas poled ceramic has a preferred crystallographic orientation and is mechanically anisotropic. If we further assume that the transformation depends not only on the magnitude of the compressive stress, but also its orienta-
\end{abstract}


tion relative to some feature(s) of PZT $95 / 5-2 \mathrm{Nb}$ 's crystallography, then these disparate results can be qualitatively resolved. In this report, we first summarize the existing results for unpoled and poled ceramic. Using our orientation-dependent hypothesis and these results, we derive simple arithmetic expressions that accurately describe our previously-observed effects of nonhydrostatic stress on the transformation of unpoled ceramic. We then go on to test new predictions based on the orientation-dependent model. It has long been known that the transformation can be triggered in uniaxial compression: the model specifically requires a steadily increasing axial stress to drive the transformation of a randomly-oriented polycrystal to completion. We show that when the stress is held constant during uniaxial compression experiments, the transformation stops, supporting our hypothesis. We close with a discussion of implications of our model, and ways to test it using poled ceramic. 


\section{Contents}

1 Introduction 1

2 Background: Evidence for a "Maximum Compressive Stress" Criterion 5

2.1 Hydrostatic Compression and CSD Experiments on PNZT: A

Brief Review . . . . . . . . . . . . . . . . 5

2.2 Evidence from Experiments on Unpoled Specimens . . . . . 7

2.3 Counterevidence and New Supporting Evidence from Experiments on Poled Ceramic . . . . . . . . . . . . . . 13

3 Experimental Materials and Technique 29

3.1 Experimental Materials . . . . . . . . . . . . . . . . 29

3.2 Experimental Apparatus and Technique ......... 31

4 Experimental Results 33

4.1 Compressive Strength and Elastic Properties . . . . . . . . . 33

4.2 Mechanical Behavior and Evidence for Occurrence of the Phase

Transformation . . . . . . . . . . . . . 38

4.3 Capacitance Measurements and Onset of the Transformation . 49

4.4 Summary of Results from Constant Strain Rate Experiments . 53

4.5 Interrupted Uniaxial Compression Experiments . . . . . . 54

5 Discussion and Conclusions $\quad 58$

6 References $\quad 60$ 


\section{List of Figures}

1.1 Pressure-temperature phase diagram for the PNZT system. . . 2

2.1 Effect of nonhydrostatic stress on mean stress for transformation of unpoled 424 ceramic, as indicated by volume strain data. ..................... 8

2.2 Capacitance-pressure signatures for hydrostatic compression experiments on unpoled 424 ceramic. . . . . . . . . . . . . . 9

2.3 Plots of pressure or mean stress against volume strain for hydrostatic compression, constant stress difference and uniaxial compression experiments, respectively, on unpoled 541-1 ceramic. ...................... 10

2.4 Plots of capacitance versus pressure or mean stress for hydrostatic compression and constant stress difference experiments, respectively, on unpoled 541-1 ceramic.

2.5 Plot of pressure or $\sigma_{1}$ versus volume strain for hydrostatic compression and constant stress difference experiments, respectively, on unpoled 424 ceramic. . . . . . . . . . . . 12

2.6 Capacitance-pressure signatures for hydrostatic compression experiments on unpoled 424 ceramic. . . . . . . . . . . 13

2.7 Effect of nonhydrostatic stress on mean stress for transformation of unbiased, poled 424 ceramic, as indicated by volume strain data. . . . . . . . . . . . . . . . . 14

2.8 Plot of discharge voltage versus pressure or mean stress for representative hydrostatic compression and CSD experiments on unbiased, poled 424 ceramic, respectively. . . . . . . . . . 16

2.9 Plot of discharge voltage versus pressure or mean stress for representative hydrostatic compression and CSD experiments on biased, poled 424 ceramic, respectively. . . . . . . . . . 17

2.10 Plot of discharge voltage versus pressure or mean stress for representative hydrostatic compression and CSD experiments on unbiased, poled 541-1 ceramic, respectively. . . . . . . . 18 
2.11 Plots of pressure or $\sigma_{1}$ versus volume strain for selected hydrostatic and CSD experiments, respectively, on unbiased, poled 424 ceramic.

2.12 Plots of discharge voltage versus pressure or $\sigma_{1}$ for hydrostatic and CSD experiments, respectively, on unbiased, poled 424 ceramic

2.13 Plots of discharge voltage versus pressure or $\sigma_{1}$ for hydrostatic and CSD experiments, respectively, on biased, poled 424 ceramic.

2.14 Plots of discharge voltage versus pressure or $\sigma_{1}$ for hydrostatic and CSD experiments, respectively, on unbiased, poled 541-1 ceramic.

2.15 Plots of pressure or $\left(\sigma_{1}+\sigma_{3}\right) / 2$ versus volume strain for hydrostatic and CSD experiments, respectively, on unbiased, poled 424 ceramic.

2.16 Plots of discharge voltage versus pressure or $\left(\sigma_{1}+\sigma_{3}\right) / 2$ for hydrostatic and CSD experiments, respectively, on unbiased, poled 424 ceramic.

2.17 Plots of discharge voltage versus pressure or $\left(\sigma_{1}+\sigma_{3}\right) / 2$ for hydrostatic and CSD experiments, respectively, on biased, poled 424 ceramic.

2.18 Plots of discharge voltage versus pressure or $\left(\sigma_{1}+\sigma_{3}\right) / 2$ for hydrostatic and CSD experiments, respectively, on unbiased, poled 541-1 ceramic.

2.19 Plots of pressure or mean stress versus volume strain for hydrostatic compression, CSD and uniaxial compression experiments on unpoled specimens from a third sintering batch, hifire 453 .

4.1 Typical uniaxial compression experiment on unpoled 424 ce-

4.2 Typical uniaxial compression experiment on unpoled 541-1 ceramic.

4.3 Representative pressure and mean stress ramping rates for hydrostatic compression, uniaxial compression and constant stress difference experiments on unpoled 424 ceramic.

4.4 Unload/reload cycles used to measure Young's modulus (E) and Poisson's ratio $(\nu)$ in early stages of a uniaxial compression experiment on 424 ceramic. . . . . . . . . . . . . . 38

4.5 Detail of Figure $4.4 \ldots \ldots 39$ 
4.6 Unload/reload cycles used to measure Young's modulus (E) and Poisson's ratio $(\nu)$ at various stages of a uniaxial compression experiment on 541-1 ceramic. . . . . . . . . 40

4.7 Detail of Figure 4.6 showing the two loops used to calculate $\mathrm{E}$ and $\nu \ldots \ldots \ldots \ldots \ldots 4 \ldots$

4.8 Enlarged plot of lateral strain behavior during uniaxial compression of 424 ceramic. . . . . . . . . . . . . . . 42

4.9 Enlarged plot of lateral strain behavior during uniaxial compression of 541-1 ceramic. . . . . . . . . . . . . . 43

4.10 Summary plot comparing volume strain behavior of five uniaxial compression experiments on unpoled 424 ceramic with three hydrostatic compression experiments on the same material. 46

4.11 Summary plot comparing volume strain behavior of five uniaxial compression experiments on unpoled 541-1 ceramic with three hydrostatic compression experiments on the same material. 47

4.12 Relative dielectric constant measurements for uniaxial compression experiments on 424 ceramic. . . . . . . . . . 51

4.13 Relative dielectric constant measurements for uniaxial compression experiments on 541-1 ceramic. . . . . . . . . 52

4.14 Plot of volume strain and $\sigma_{1}$ versus time for unconfined, constant strain rate experiment on 424 ceramic in which displacement was interrupted three times.

4.15 Plot of volume strain and $\sigma_{1}$ versus time for unconfined, constant strain rate experiment on 424 ceramic in which displacement was interrupted three times.

4.16 Plot of volume strain and $\sigma_{1}$ versus time for unconfined, constant strain rate experiment on 424 ceramic in which displacement was interrupted three times. 


\section{List of Tables}

4.1 Uniaxial compression experiments on unpoled hifire 424 ceramic. $\sigma_{1_{f}}$ and $\epsilon_{v_{f}}$ are the compressive stress and volume strain at failure, respectively. Strain rate was $1.0 \times 10^{-4} \mathrm{~s}^{-1}$. 37

4.2 Uniaxial compression experiments on unpoled, hifire 541-1 ceramic. $\sigma_{1_{f}}$ and $\epsilon_{v_{f}}$ are the compressive stress and volume strain at failure, respectively. Strain rate was $1.0 \times 10^{-4} \mathrm{~s}^{-1}$. 37 


\section{Chapter 1}

\section{Introduction}

PZT 95/5-2Nb, $\mathrm{Pb}_{0.99} \mathrm{Nb}_{0.02}\left(\mathrm{Zr}_{0.95}, \mathrm{Ti}_{0.05}\right)_{0.98} \mathrm{O}_{3}$, is an interesting modification of lead-zirconate-titanate that is used in shock-activated power supplies. ${ }^{1,2}$ At room temperature, PZT $95 / 5-2 \mathrm{Nb}$ (referred to hereafter as "PNZT") has a rhombohedrally distorted perovskite structure and is ferroelectric (Figure 1.1). ${ }^{3}$ Because the material is ferroelectric, it may be placed in an electric field and "poled." That is, its unit cell-scale dipoles, oriented along any one of the eight [111] "cubic" directions, align spontaneously with the electric field insofar as mechanically possible. When the field is relaxed, some permanent polarization and a bound surface charge remain. ${ }^{1-5}$

At a pressure of $250-300 \mathrm{MPa}$ the poled rhombohedral phase $\left(F_{R 1}\right)$ undergoes a displacive, weak first-order ${ }^{4,6}$ polymorphic transformation to an antiferroelectric, orthorhombic $\left(A_{O}\right)$ phase (Figure 1.1). ${ }^{3}$ The transformation is rapid though hysteretic, characterized by a $0.7-0.8 \%$ volume decrease and changes in dielectric properties. ${ }^{3,7-11}$ Though also polar on the unit cellscale, the $A_{O}$ phase is macroscopically electrically neutral, and the previously bound charge is released. If the transformation takes place under shock wave conditions, extremely high currents and voltages can be obtained. ${ }^{1,2}$

In support of an ongoing program to develop mathematical models for the transformation, ${ }^{e . g .5}$ and 3-D numerical simulations of the operation of shock-activated power supplies, we have conducted an extensive experimental investigation of the electromechanical behavior of PNZT ceramic across the $F_{R 1} \rightarrow A_{O}$ phase boundary. ${ }^{7-11}$ The objective of this program has been to develop a constitutive model for PNZT that describes behavior before, during and after the transition. The stress state under shock conditions is not hydrostatic, and deviatoric stresses are known to often have profound effects on polymorphic transformations. (See, for instance, Larché ${ }^{12}$ and Shimizu ${ }^{13}$ for fairly recent reviews of the materials science and earth science literatures, respectively.) Coe and Paterson ${ }^{14}$, for example, demonstrated that nonhydro- 


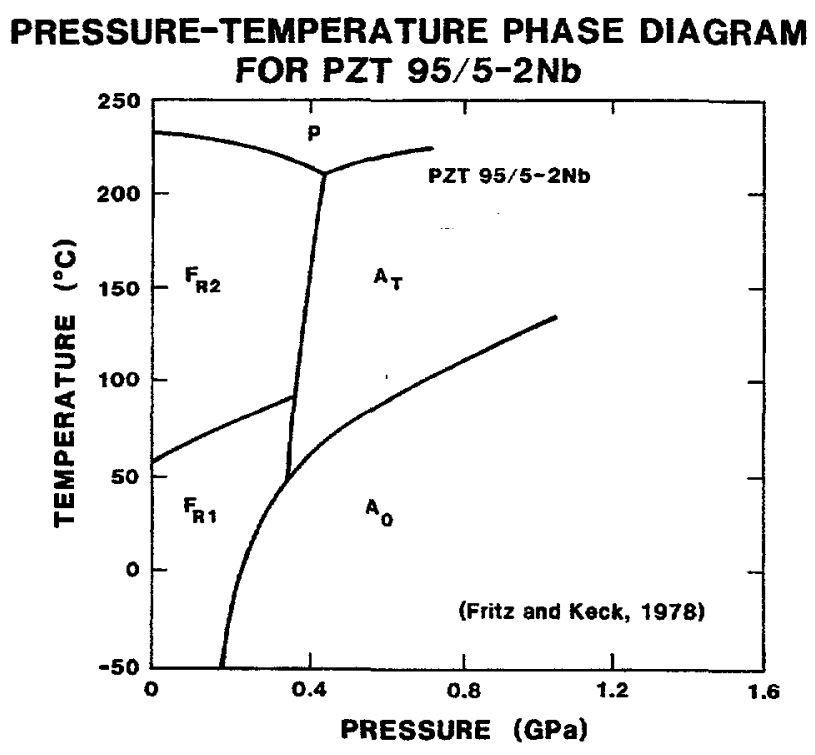

Figure 1.1: Pressure-temperature phase diagram for the PNZT system. ${ }^{3}$ The $F_{R 1} \rightarrow A_{O}$ transformation is the subject of this paper. Note that there are three additional, known polymorphs at higher temperatures: a rhombohedral, ferroelectric phase, $\left(F_{R 2}\right)$, a tetragonal, antiferroelectric, $\left(A_{T}\right)$ phase, and a cubic, paraelectric phase $(P)$.

static loading increased the temperature for onset of the displacive $\alpha \rightarrow \beta$ quartz transformation; they further showed that the orientation between stress and quartz's crystal structure also affects the transition temperature. Thus, we have been particularly interested in the effects of deviatoric loading on the transformation, albeit at constant temperature. Our principal tool in this investigation has therefore been the constant stress difference (CSD) experiment, in which we apply a constant shear stress while increasing the compressive mean stress to cross the transformation boundary. ${ }^{7-11}$

Our earliest experiments were performed on unpoled PNZT ceramic. ${ }^{7-9}$ Using hydrostatic and triaxial compression experiments at room temperature, $\left(\approx 20^{\circ} \mathrm{C}\right)$, we showed that application of increasing shear stresses, which caused only elastic strains: (1) systematically lowered the mean stress at which the transformation occurred, (2) caused anisotropic strain behavior during the transformation, and (3) slowed the rate at which the transformation took place for a constant pressurization rate. We also found limited evidence for the shape-memory effect. ${ }^{7,8}$ Subsequently, we presented convincing evidence that under nonhydrostatic compression, the transformation 
occurred when the maximum compressive stress equalled the hydrostatic pressure at which the transformation would otherwise occur. ${ }^{9}$ (Hereafter, we will refer to the transition pressure for unpoled ceramic as $P_{T}^{U}$, and that for poled ceramic as $P_{T}^{P}$.) If correct, this criterion is quite important because a realistic model for phase equilibrium in the PNZT system should necessarily predict this result for a general stress state.

More recently, we examined the behavior of poled PNZT in hydrostatic and triaxial compression, with and without an applied electric field. ${ }^{10,11}$ In the constant stress difference experiments, the maximum compressive stress was applied perpendicular to the poling direction. We showed (as expected) ${ }^{4}$ that the hydrostatic pressure for transformation of poled ceramic was somewhat higher than that for unpoled. We also found weak evidence that an applied electric field raised the hydrostatic pressure for onset of the transformation. Though we detected no evidence for a shape-memory effect, we again found that poled ceramic behaved identically to unpoled ceramic with respect to items (1)-(3) listed in the preceding paragraph. The apparent kinetic effect was particularly important, because it was directly linked to decreased discharge voltages and voltage rates. Our results suggested that power supply performance could be enhanced by finding a way to reduce deviatoric stresses during shock loading. ${ }^{10,11}$

Disappointingly, however, we were unable to confirm the transformation criterion that we had established for unpoled ceramic: the maximum compressive stresses at which the transformation occurred in poled ceramic were always shifted to higher values than the hydrostatic pressure at which the transformation would otherwise take place. We will subsequently refer to the hydrostatic pressure for transformation of poled ceramic as $P_{T}^{P}$.

We noted at the time, however, that poled PNZT ceramic is anisotropic, whereas unpoled ceramic is not, ${ }^{10}$ and that some sort of additional "structure factor" might need to be considered. We observed that if transformation depends not only on the magnitude of the compressive stress, but on its orientation relative to some crystallograpic surface or axis, this particular discrepancy might be qualitatively explained, along with some other effects of nonhydrostatic stress on the transition. ${ }^{11}$ We also discussed some preliminary results from uniaxial compression experiments on unpoled PNZT ceramic, which supported our modified hypothesis, and discussed additional ways to test our qualitative model. . $^{11,15}$

In this paper, we will briefly review our evidence that occurrence of the transformation depends critically on the magnitude of the maximum compressive stress, and present some new evidence from our experiments on poled ceramic that the transformation depends upon stress orientation, as well. Assuming that the hypothesis is correct, we will develop simple equations that 
quantitatively relate the applied shear stress to our observed depression of the mean stress and confining pressure at which the transformation of unpoled ceramic occurs. We will also show qualitatively how the hypothesis reasonably explains the apparent effect of nonhydrostatic stress on transformation kinetics that we have previously reported. Following this background review and development of new evidence from existing data, we will discuss some new experimental evidence from uniaxial compression tests that supports our modified hypothesis.

Fritz ${ }^{6}$ has shown that the $F_{R 1} \rightarrow A_{O}$ transformation can be induced in simple uniaxial compression experiments. In that same paper, ${ }^{6}$ he also suggested that the transformation might depend on the relationship between domain orientation and stress magnitude, but did not further examine this concept. We do so here. Our modified hypothesis makes several predictions about transformation effects that should occur during uniaxial compression of unpoled ceramic. Specifically, for uniaxial compression of a randomly oriented polycrystal: (1) the transformation should begin when the compressive stress, $\sigma_{1}$, equals $\mathrm{P}_{T}^{U}$, and (2) a steadily increasing compressive stress should be required to drive the transformation to completion. The reasons for these predicted phenomena will be discussed elsewhere in the paper.

To test these predictions, we have performed fourteen uniaxial compression experiments on unpoled ceramic from two sintering batches (subsequently referred to as "hifires") that we have investigated previously. Eleven of these experiments were run at constant strain rate, usually to failure. Volume strain data support our prediction that the transformation begins when $\sigma_{1}=\mathrm{P}_{T}^{U}$. For reasons that are not clear, the dielectric measurements are ambiguous, but, we believe, do not contradict this conclusion. Three of the experiments were done at constant strain rate, but displacement was halted at three points above the transformation stress. In most instances, the volumetric strain rate, a measure of the transformation rate, dropped by 2 orders of magnitude while the stress remained approximately constant. When deformation was resumed and the stress began to increase, the volumetric strain rate resumed at its previous value. This result also supports our hypothesis.

Following the presentation of this new experimental evidence, we will close with a discussion of some of the implications of this model and additional experiments that could be performed on both poled and unpoled ceramic to further confirm or refute our model. 


\section{Chapter 2}

\section{Background: Evidence for a "Maximum Compressive Stress" Criterion}

\subsection{Hydrostatic Compression and CSD Ex- periments on PNZT: A Brief Review}

Before proceeding to review and expand upon our previous results, we must make several remarks on terminology and our earlier experimental techniques. Our earlier methods are actually very similar to those used in the uniaxial compression tests insofar as specimen preparation, apparatus, instrumentation, data acquisition and reduction are concerned. These are discussed in great detail elswhere in this paper. However, a few brief comments now will greatly clarify the nature of the hydrostatic compression and CSD experiments that form the basis for so much of the discussion immediately at hand.

Test specimens were $1.080-\mathrm{cm}$ square by $2.540-\mathrm{cm}$ long, right parallelepipeds, which came from the same two hifires on which we have performed the uniaxial compression experiments discussed in a later section, 424 and 541-1. Silver electrodes were evaporated onto two opposing rectangular faces of the unpoled specimens to permit capacitance measurements if desired..$^{7,8}$

For poled specimens, ${ }^{10,11}$ these same electrodes were used for the actual poling operation, and, hence, the poling direction was normal to the two electroded faces, and to the axis of compression. The latter specimens were "hot-poled" in electric fields corresponding to $50 \mathrm{~V}$ per $0.003 \mathrm{~cm}(0.001 \mathrm{in})$ of thickness. Specimens were heated in oil to $105^{\circ} \mathrm{C}$, and the field applied. The temperature was then lowered to $70^{\circ} \mathrm{C}$ over a period of 5 minutes, whereupon 
the field was removed. Cooling to room temperature then continued. To permit capacitance measurements on poled specimens an additional, small set of electrodes was painted on to the faces at $90^{\circ}$ to the poling electrodes. This measurement was seldom performed on poled specimens, owing to the limited number of electrical feedthroughs available in our high pressure test cell. ${ }^{10}$

Experiments were conducted in a standard, liquid-medium triaxial testing apparatus equipped with a $1000 \mathrm{MPa}$ pressure vessel and 1.78 MN hydraulic actuator. Vessel and actuator are yoked together in a stiff reaction frame that allows transfer of force from the actuator to the specimen using a moveable piston that enters they cylindrical pressure vessel through one end closure.

Hydrostatic compresson experiments are straightforward and should require no explanation. The CSD experiments were conceived to observe the effects of constant shear stress on the transformation. In these experiments, the specimens were first pressured hydrostatically (i.e., $\sigma_{1}=\sigma_{2}=\sigma_{3}$ ) to $69 \mathrm{MPa}$ to reduce the likelihood of microcracking upon application of the deviatoric load. Here, $\sigma_{1}, \sigma_{2}$, and $\sigma_{3}$ are the greatest, intermediate and least principal stresses, respectively, compressive stresses (and strains) reckoned positive, following the usual rock mechanics convention.

Using the movable piston, an additional load was then superimposed parallel to the specimen's long axis to increase the stress difference, $\sigma_{1}-\sigma_{3}$, to $50,100,150$ or $200 \mathrm{MPa}$. In our test configuration then, the axial compressive stress is $\sigma_{1}$, and $\sigma_{2}=\sigma_{3}=$ the fluid pressure. (The fluid pressure is often referred to as the confining pressure in the rock mechanics literature.) The axial stress, $\sigma_{1}$, and the fluid pressure, $\sigma_{3}$ were then increased simultaneously under microprocessor control such that the stress difference, $\sigma_{1}-\sigma_{3}$, remained constant while the mean stress $\left(\sigma_{1}+2 \sigma_{3}\right) / 3$ increased across the phase boundary. Note that the stress difference is twice the maximum resolved shear stress on the specimen, which also, therefore, remained constant during the transformation. All specimens experienced the same constant rate of increasing mean stress, $0.55 \mathrm{MPa} \mathrm{s}^{-1}$.

Data that were collected during the experiments included axial and lateral strains, capacitance, axial load and fluid pressure. In the case of poled specimens, the discharge voltage at the transformation was also measured in a variety of ways. ${ }^{10,11}$ Details of the methods, accuracy and resolution of these measurements are given elsewhere. ${ }^{7,8,10,11}$ One experimental detail that will be mentioned below, however, is correction of the axial load, and, hence, $\sigma_{1}$, for piston friction. The high-pressure seal in the pressure vessel end closure exerts a pressure-dependent friction on the moveable piston that passes through it. Uncorrected for, this results in an overestimate of the actual axial stress on the specimen. Referred to a specimen of our cross- 
sectional area, this correction to $\sigma_{1}$ can range from negligible at $\sigma_{3}=0$ to 33 $\mathrm{MPa}$ at $\sigma_{3}=500 \mathrm{MPa}$. The effects become nonlinear above about $350 \mathrm{MPa}^{9}$ For experiments performed at a constant confining pressure, the friction can be measured from load-piston displacement hysteresis loops. The value of friction can then be subtracted from the force record, correcting $\sigma_{1}$. Our experiments are conducted at pressures that change continuously up to 500 $\mathrm{MPa}$. Hence, piston friction also changes continuously. making it very difficult to correct the entire force record. Thus, where we have corrected axial loads in the past, ${ }^{9}$ the corrections have been made at specific pressures that correspond to a single instant in time and a single set, or line, of data.

\subsection{Evidence from Experiments on Unpoled Specimens}

We have shown previously that increasing shear stresses lower both the mean stress and confining pressure for onset of the transformation of unpoled ceramic based on both volume strain and capacitance measurements (Figures 2.1-2.4) ${ }^{7-9}$ Subsequently, we reexamined some of those same data ${ }^{9}$ in terms of maximum compressive stress, $\sigma_{1}$, versus both volume strain and the maximum capacitance reached in a test, having shown that the latter quantity had a very good correlation with onset of the transformation as indicated by the volume strain.

Owing, perhaps, to our inability to correct the complete force record for piston friction, the plots of $\sigma_{1}$ versus volume strain yielded an ambiguous answer (Figure 2.5). Onset of the the transformation as indicated by the volume strain record appeared to be shifted to values of $\sigma_{1}$ somewhat higher

than the $\mathrm{P}_{T}^{U}$ indicated by hydrostatic compression tests. However, this upward shift was in the range of the overestimates of $\sigma_{1}$ that piston friction would cause at the transformation confining pressures. Initially, plots of $\sigma_{1}$ versus peak (maximum) capacitance showed a similar shift, but we could readily examine the effect of piston friction in this latter case.

The peak capacitances could be picked unambiguously from each test record. The values of $\sigma_{1}$ corresponding to those points were then identified and corrected for piston friction, which we have measured over the range of relevant pressures. ${ }^{9}$ The data were plotted for comparison with the results of the hydrostatic compression tests, which required no correction. The resulting correlation is shown in Figure 2.6. Though not perfect, it seems clear that the onset for transformation under nonhydrostatic stress (as indicated by the peak capacitance) occurs when $\sigma_{1}=P_{T}^{U}$.

Working from this assumption, and considering the manner in which our 


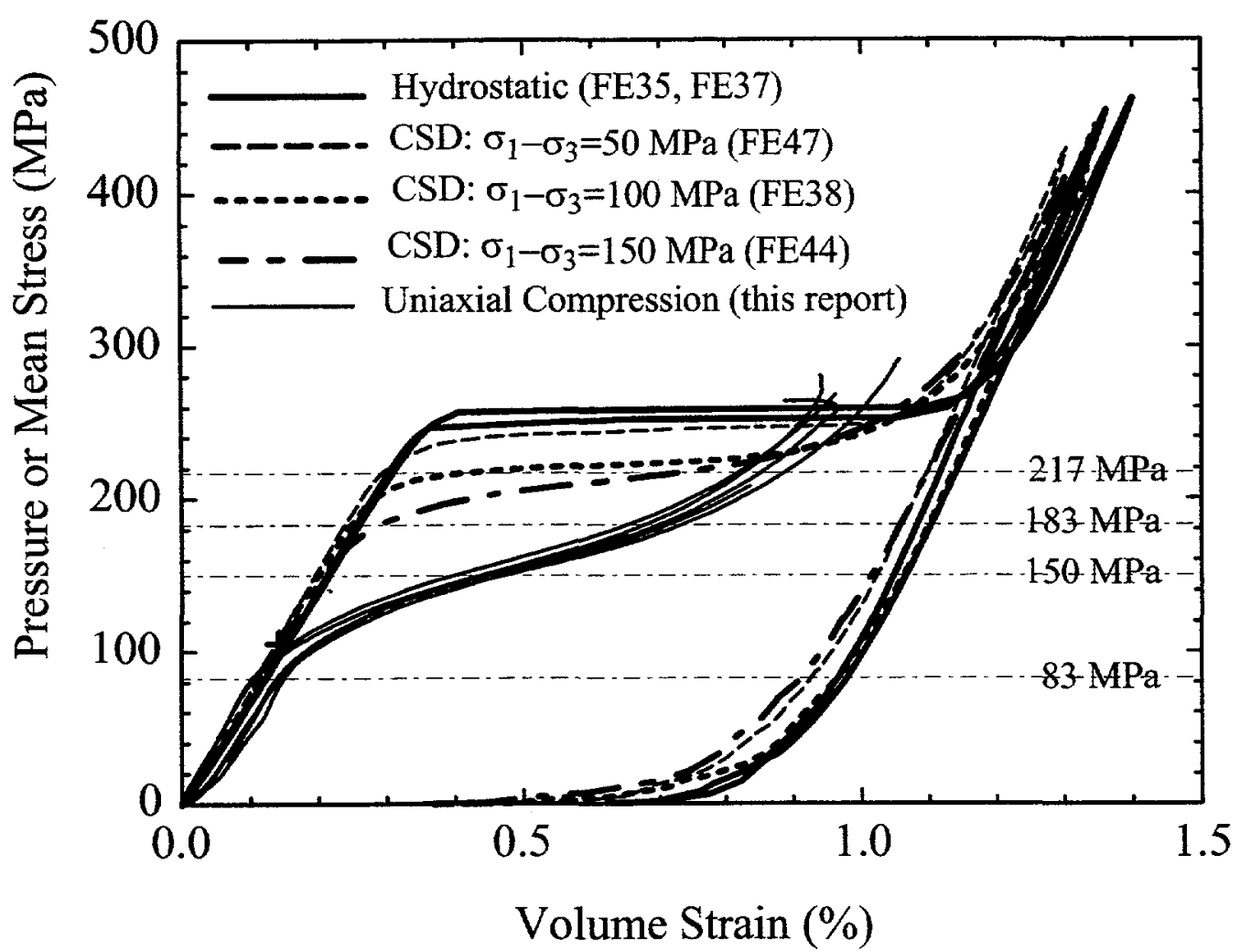

Figure 2.1: Effect of nonhydrostatic stress on mean stress for transformation of unpoled 424 ceramic, as indicated by volume strain data. Sense of increasing pressurization is clockwise. Sharp volume decrease at $\mathbf{P}=\mathbf{2 5 0} \mathrm{MPa}$ for hydrostatic compression tests indicates onset of the transformation. With increasing stress difference, the mean stress for onset of the transformation is depressed. Horizontal lines indicate the mean stresses for onset of the transformation predicted from Eq. (2.2) for uniaxial compression and CSD tests.

CSD experiments are performed, it is a simple arithmetic exercise to show that for a constant stress difference, $\sigma_{d}=\sigma_{1}-\sigma_{3}$, the transformation will begin at a confining pressure,

$$
\sigma_{3}=P_{T}^{U}-\sigma_{d}
$$

and a mean stress 


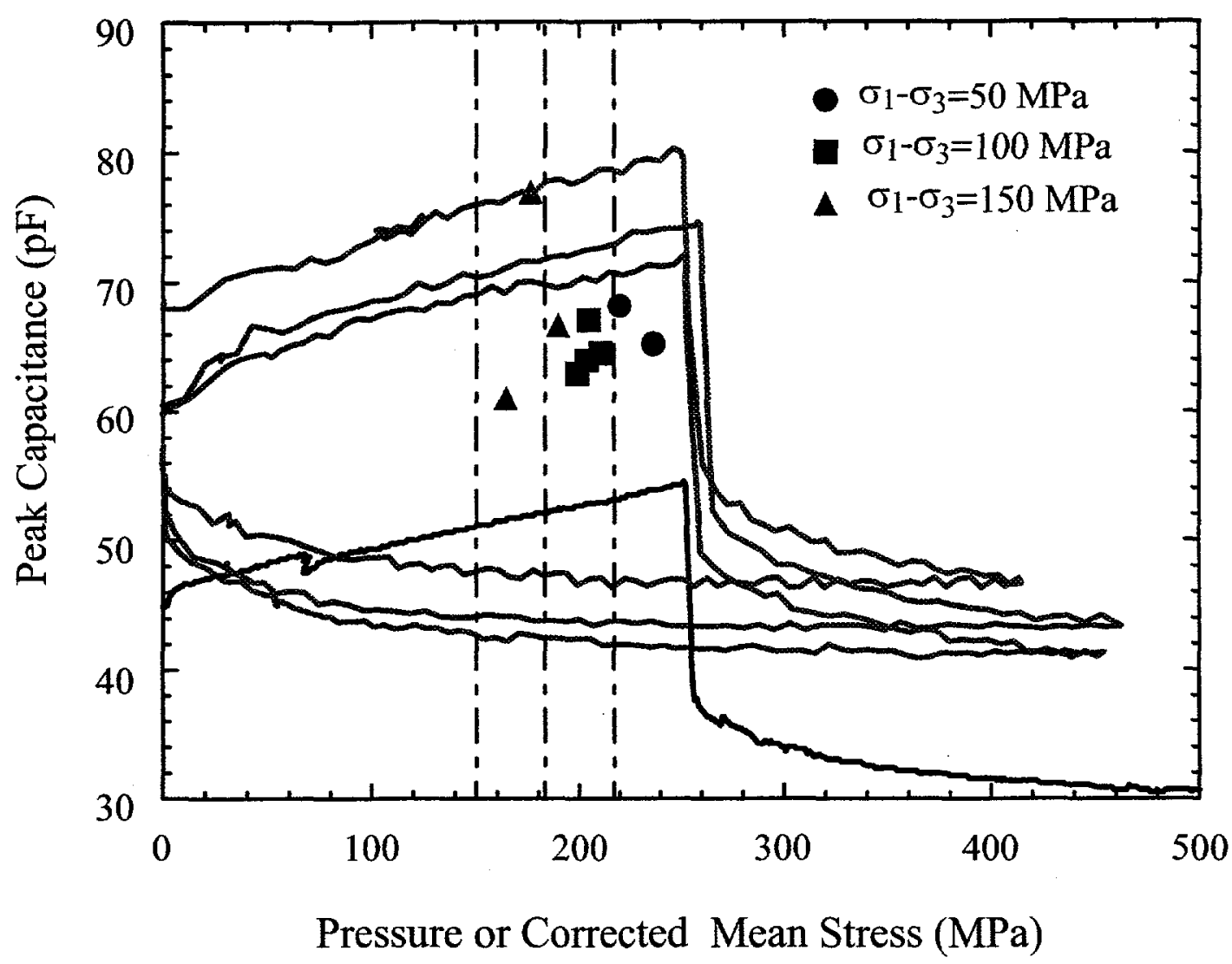

Figure 2.2: Solid lines are capacitance-pressure signatures for hydrostatic compression experiments on unpoled 424 ceramic. Sense of increasing pressurization is clockwise. Sharp drops at about $250 \mathrm{MPa}\left(P_{T}^{U}\right)$ indicate the $F_{R 1} \rightarrow A_{O}$ transformation. Solid symbols represent the peak capacitances of constant stress difference experiments plotted against mean stress. Like the volume strain data, capacitance data indicate depression of the mean stress for onset of the transformation with increasing shear stress.

$$
\sigma_{m}=\frac{3 P_{T}^{U}-2 \sigma_{d}}{3} .
$$

For our CSD experiments on unpoled 424 ceramic, $P_{T}^{U}=250 \mathrm{MPa}$, and $\sigma_{d}=50,100$ or $150 \mathrm{MPa}$. Use of Eq. (2.2) "predicts" corresponding mean stresses for onset of the transformation to be 217,183 and $150 \mathrm{MPa}$, respectively. These values are indicated as dotted horizontal and vertical lines on Figures 2.1 and 2.2, respectively. Although agreement is not perfect, it 


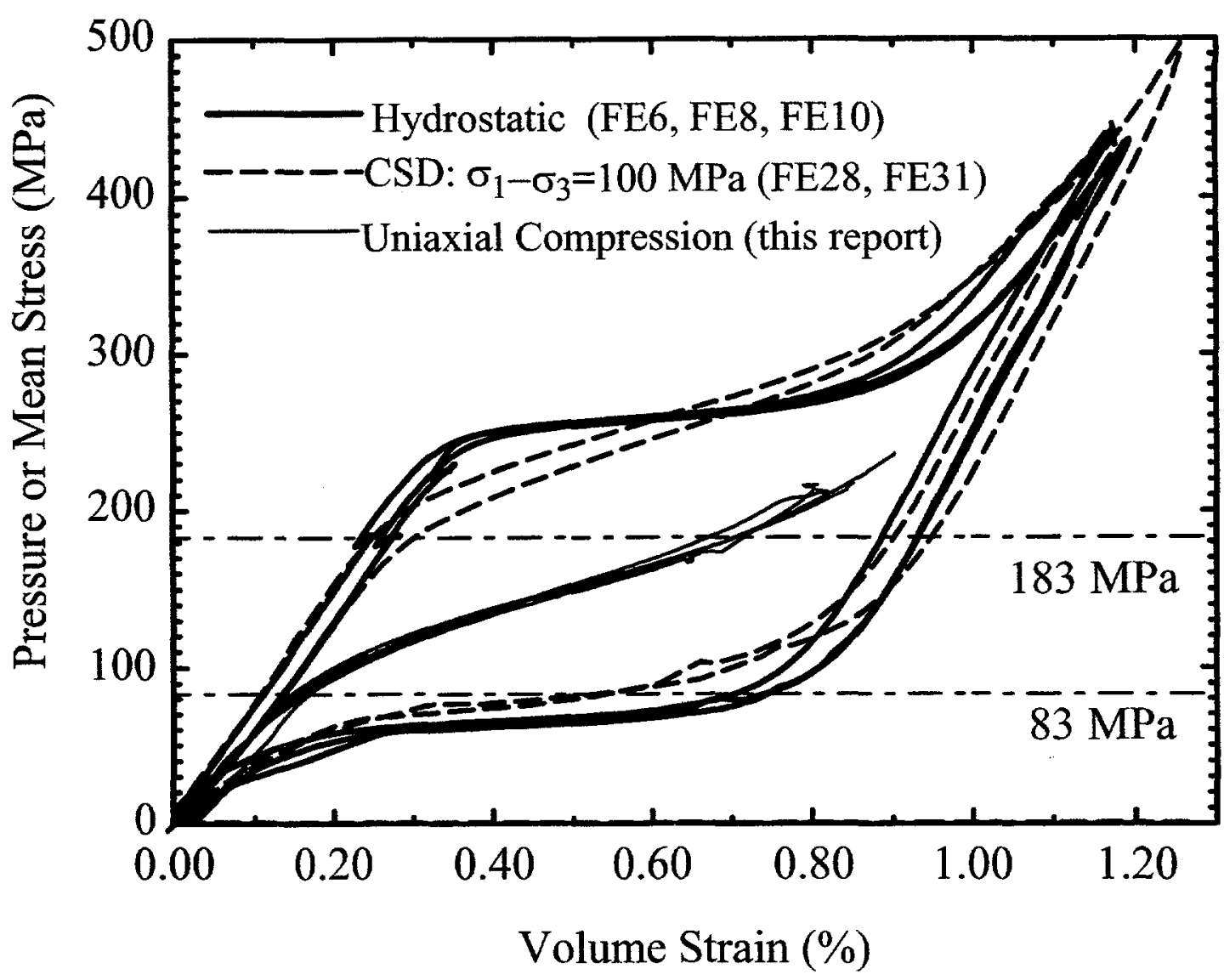

Figure 2.3: Plots of pressure or mean stress against volume strain for hydrostatic compression, constant stress difference and uniaxial compression experiments, respectively, on unpoled 541-1 ceramic. Sense of pressurization is clockwise. Sharp drops at about $250 \mathrm{MPa}$ $\left(P_{T}^{U}\right)$ indicate the $F_{R 1} \rightarrow A_{O}$ transformation.Horizontal lines indicate the mean stresses for onset of the transformation predicted from Eq. (2.2) for uniaxial compression and CSD experiments. Note that in hydrostatic and triaxial compression, the transformation of 541-1 ceramic is usually completely reversed.

is clear that Eq. (2.2) quantitatively captures the effect of shear stress on the mean stress for transformation as determined from both volume strain and peak capacitance data. Comparisons between "predictions" based on Eq. (2.1) and actual confining pressures for transformation ${ }^{7, A p p . B}$ are equally good.

Note that if our assumption that the transformation begins when $\sigma_{1}=P_{T}^{U}$ 


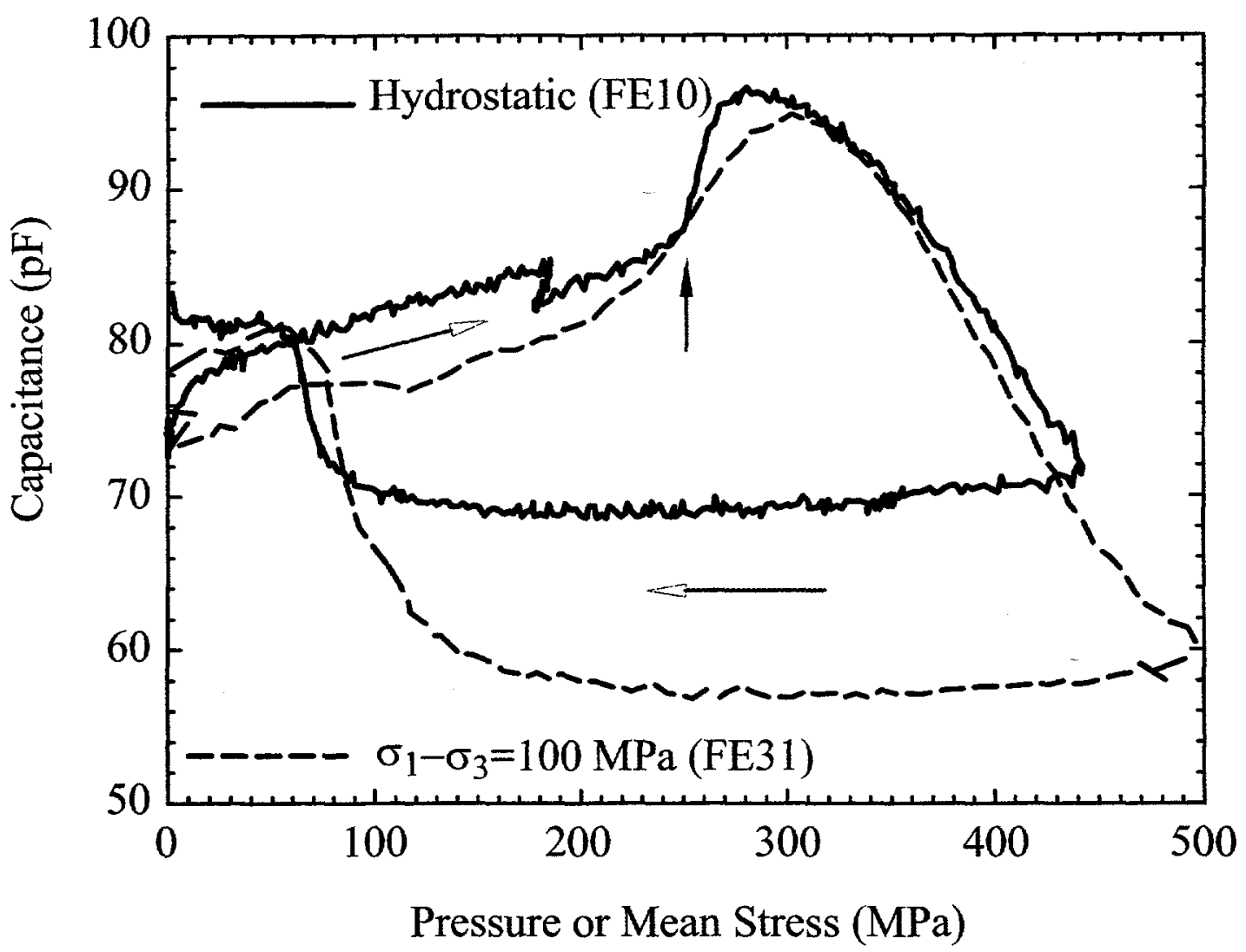

Figure 2.4: Plots of capacitance versus pressure or mean stress for hydrostatic compression and constant stress difference experiments, respectively, on unpoled 541-1 ceramic. Sense of pressurization is clockwise (open arrows). Note the marked increase in capacitance at the transformation in the hydrostatic test (filled arrow), unlike the sharp drop exhibited by 424 ceramic. Note also the smearing out of the capacitance signature at a stress difference of $100 \mathrm{MPa}$.

is correct, we can make two predictions about when the transformation should occur in a uniaxial compression experiment on 424 ceramic (i.e., $\sigma_{3} \approx 0$ ). Quite simply, the transformation should start when $\sigma_{1}=250 \mathrm{MPa}$. The mean stress for onset of the transformation should therefore be $83 \mathrm{MPa}$, the lowest that we have ever observed. We will test these predictions later in this paper.

Returning to Figure 2.1, it is important to note the apparent effect of shear stress on the kinetics of the transformation. As noted above, all specimens are pressurized at the same rate. With increasing shear stress, the 


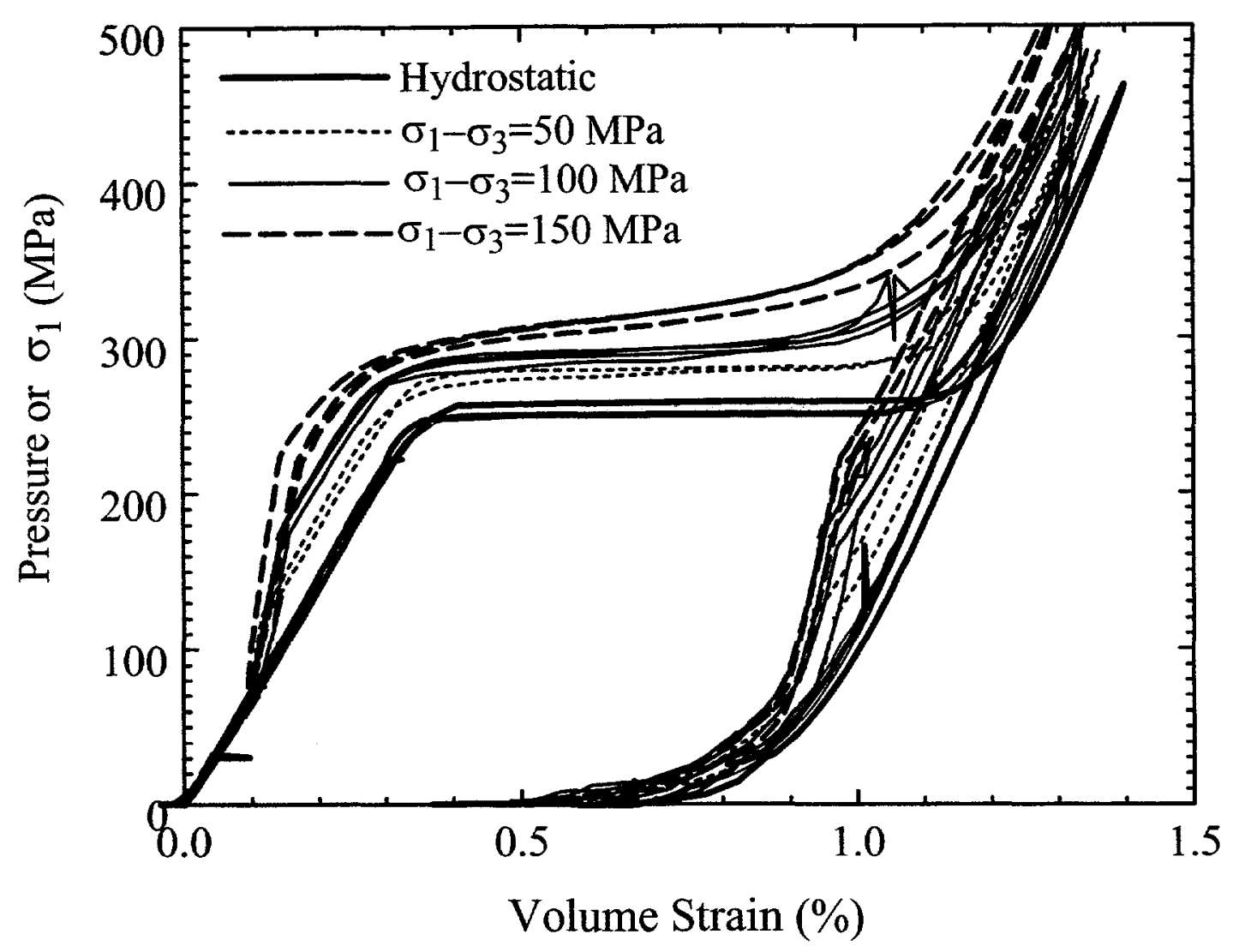

Figure 2.5: Plot of pressure or $\sigma_{1}$ versus volume strain for hydrostatic compression and constant stress difference experiments, respectively, on unpoled 424 ceramic. Offsets in $\sigma_{1}$ for CSD experiments at $69 \mathrm{MPa}$ occur when deviatoric load is applied. Although values of $\sigma_{1}$ at which transformation starts appear to be higher than the hydrostatic transformation pressure, $\sigma_{1}$ has not been corrected for piston seal friction and should actually be lower.

transformation begins at lower mean stress. However, as indicated by the greater radii of curvature of the mean stress-volume strain plots at higher shear stresses, the transformation also takes longer to complete. Thus, for a constant rate of pressurization, increasing shear stresses retard the rate of transformation. We have shown previously that this also affects the rate of charge release and peak voltage that are attained during transformation of poled ceramic. ${ }^{10,11}$ Until now, we have not had a fully satisfactory explanation for this particular observation. We will further describe and test this explanation later. 


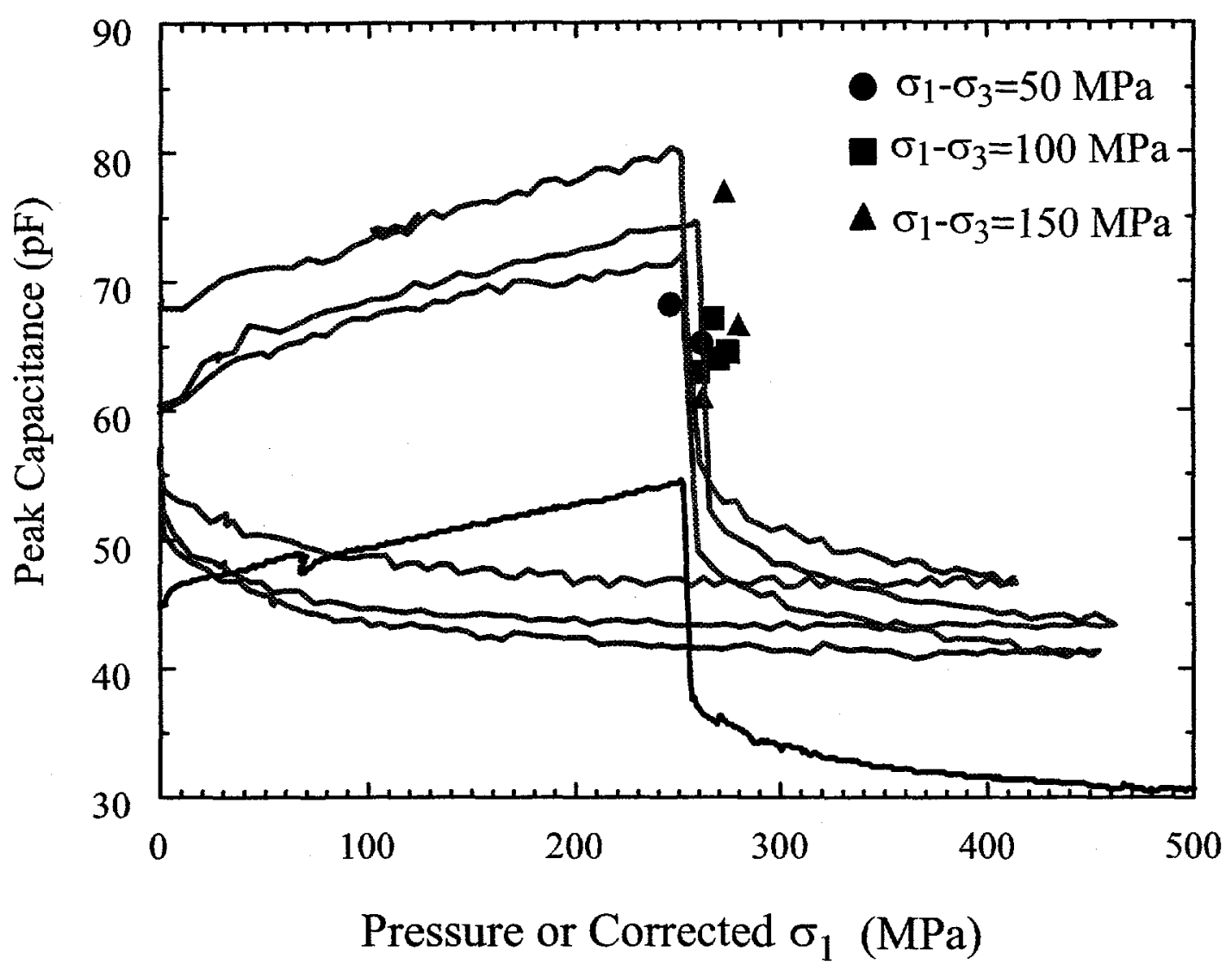

Figure 2.6: Solid lines are capacitance-pressure signatures for hydrostatic compression experiments on unpoled 424 ceramic. Sense of increasing pressurization is clockwise. Sharp drops at about 250 MPa $\left(P_{T}^{U}\right)$ indicate the $F_{R 1} \rightarrow A_{O}$ transformation. Solid symbols represent the peak (maximum) capacitances of constant stress difference experiments plotted against $\sigma_{1}$, which has been corrected for piston seal friction. ${ }^{9}$ Note close correspondence between the corrected values of $\sigma_{1}$ and $P_{T}^{U}$.

\subsection{Counterevidence and New Supporting Ev- idence from Experiments on Poled Ce- ramic}

In Figure 2.7 we present mean stress-volume strain plots for hydrostatic compression and CSD experiments on poled 424 ceramic. ${ }^{10,11}$ Although the 


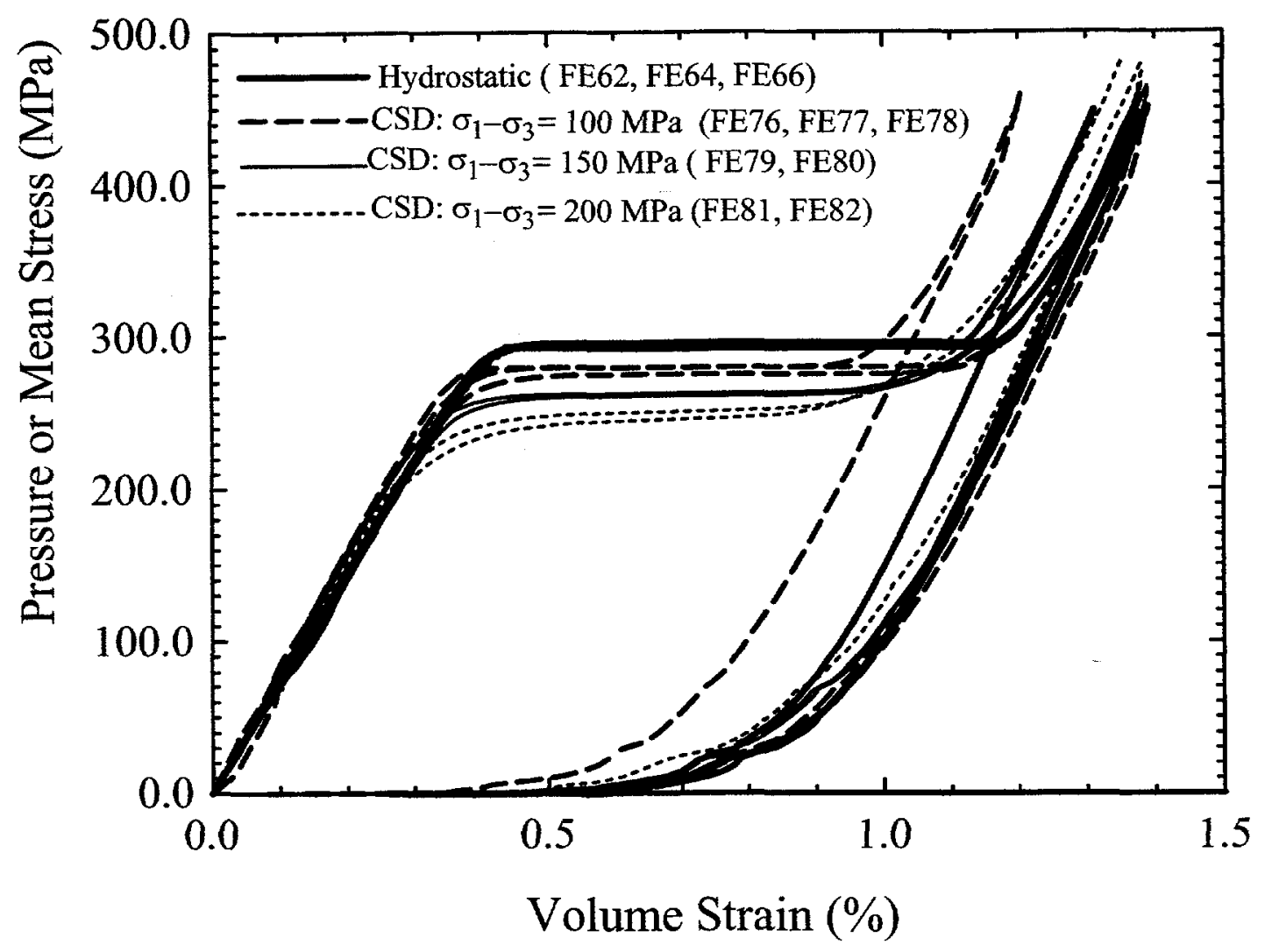

Figure 2.7: Effect of nonhydrostatic stress on mean stress for transformation of unbiased, poled $\mathbf{4 2 4}$ ceramic, as indicated by volume strain data. ${ }^{10,11}$ Sense of increasing pressurization is clockwise. Sharp volume decrease at $\mathrm{P}=300 \mathrm{MPa}\left(P_{T}^{P}\right)$ for hydrostatic compression tests indicates onset of the transformation. With increasing stress difference, the mean stress for onset of the transformation is depressed. Note that the hydrostatic transformation pressure for poled ceramic, $P_{T}^{P}$, is greater than that for unpoled ceramic, $P_{T}^{U}$ (cf. Figure 2.1).

hydrostatic pressure for transformation of poled ceramic, $\mathrm{P}_{T}^{P}$, is greater than that for unpoled ceramic, $\mathrm{P}_{T}^{U}$, the results are qualitatively the same as for unpoled material. Mean stress and confining pressure for transformation decrease with increasing shear stress. Transformation rate also decreases with increasing shear stress. Results from a few experiments on poled 541-1 ceramic were qualitatively identical to those for 424 material, although our strain measurements were less complete. ${ }^{10}$ 
Owing to a shortage of feedthroughs in our high pressure vessel, capacitance measurements were conducted on only a few poled specimens from hifire $541-1 .^{10}$; in subsequent experiments on 424 ceramic, we chose to make more complete strain measurements, at the expense of capacitance measurments. However, we did monitor discharge voltages during all of our experiments on poled ceramic. Evolving experience and differing experimental conditions (e.g., measuring discharge voltages while maintaining a $1 \mathrm{kV}$ bias on some specimens) unfortunately resulted in three different configurations for the same measurement. ${ }^{10,11}$ But despite differences in the monitoring configurations, we showed that the discharge voltages correlated closely with the transformation as indicated by volume strains. Plots of discharge voltage versus mean stress for the three different experimental configurations, and two different hifires, are shown in Figure 2.8-2.10. Note first the diminished peak voltages and more spread out voltage signatures with increasing shear stress, reflecting the kinetic effect.

Like the volume strain data (Figure 2.7), the discharge voltage data reflect a reduction in mean stress for onset of the transformation with increasing shear stress (Figure 2.8-2.10). As we did with the data for unpoled ceramic, we replotted volume strain and discharge voltage against $\sigma_{1}$ in Figure 2.11 and 2.12-2.14, respectively. ${ }^{10,11}$ In these plots, however, the values of $\sigma_{1}$ at which the transformation occurs are, in some instances, almost $100 \mathrm{MPa}$ higher than the hydrostatic pressure for transformation, $\mathrm{P}_{T}^{P}$. Shifts of this magnitude are too high to attribute to effects of piston seal friction on $\sigma_{1}$, which probably shouldn't be greater than about $35 \mathrm{MPa}$, even at a confining pressure of $500 \mathrm{MPa}$. This result required that we either abandon our earlier hypothesis, that the transformation begins when $\sigma_{1}=P_{T}^{U}$ or $P_{T}^{P}$, or modify it it somehow to include the behavior of poled ceramic.

Poling aligns as many dipoles as is mechanically possible transverse to the axis of compression. This represents a real, if imperfect, crystallographic preferred orientation, resulting in measurable differences in mechanical properties with direction. ${ }^{6,10}$ If the transformation depends not only on the magnitude of the compressive stress, but its orientation relative to some crystallographic plane(s) or direction(s) of the PNZT structure, then this abnormal shift might still be explicable within the context of our earlier assumption, that transformation begins when $\sigma_{1}=P_{T}^{P}$. This constitutes our "modified hypothesis." For example, in order to resolve a required critical normal stress on a plane inclined at some angle to the axis of compression (other than $90^{\circ}$ ) always requires an axial stress greater than the critical stress. This is clear from a simple Mohr circle construction in two dimensions. ${ }^{16, p .73}$

Exploring this possibility, we replotted the volume strain and discharge voltage data of Figures 2.7 and 2.8-2.10 against the compressive normal stress 


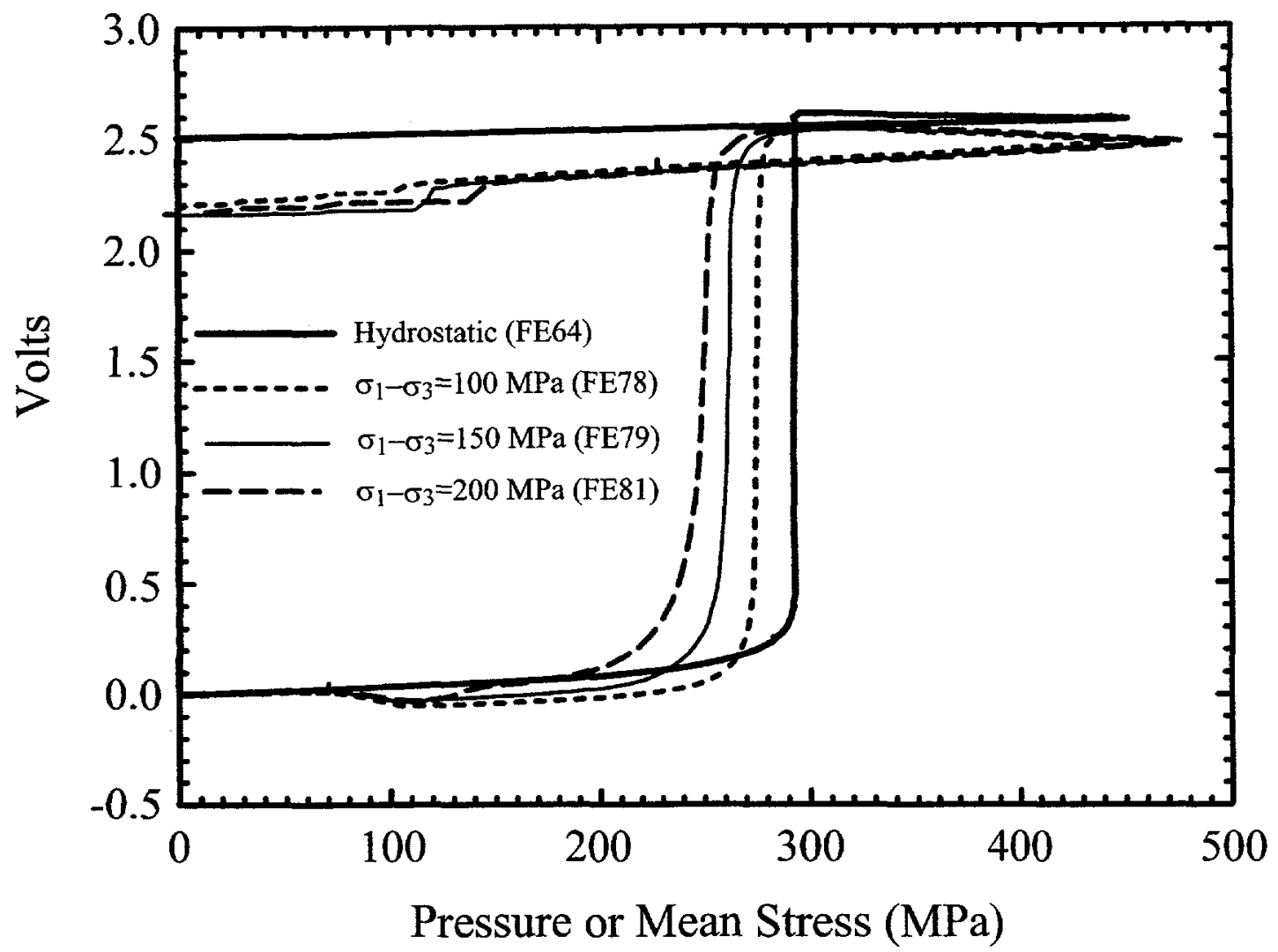

Figure 2.8: Plot of discharge voltage versus pressure or mean stress for representative hydrostatic compression and CSD experiments on unbiased, poled 424 ceramic, respectively. ${ }^{10,11}$ Note the depression of the mean stress for onset of transformation with increasing stress difference. Increased radii of curvature indicate a slowing of the transformation rate with increasing stress difference.

resolved on a plane inclined at $45^{\circ}$ to $\sigma_{1}, \frac{\sigma_{1}+\sigma_{3}}{2}$ (Figures 2.15 and 2.16-2.18). In each instance, results from the various CSD experiments on poled ceramic are brought into remarkable "registry" with the results from the hydrostatic compression experiments.

The results shown in Figures 2.15-2.18 qualitatively support our contention that the orientation, as well as the magnitude of the stress are important for onset of the transformation. Quantitively, however, it is not yet clear that any conclusions can be drawn from this result because the preferred orientation induced by poling is imperfect, and can be characterized only statistically. Thus, the results shown in Figures 2.15-2.18 cannot be in- 


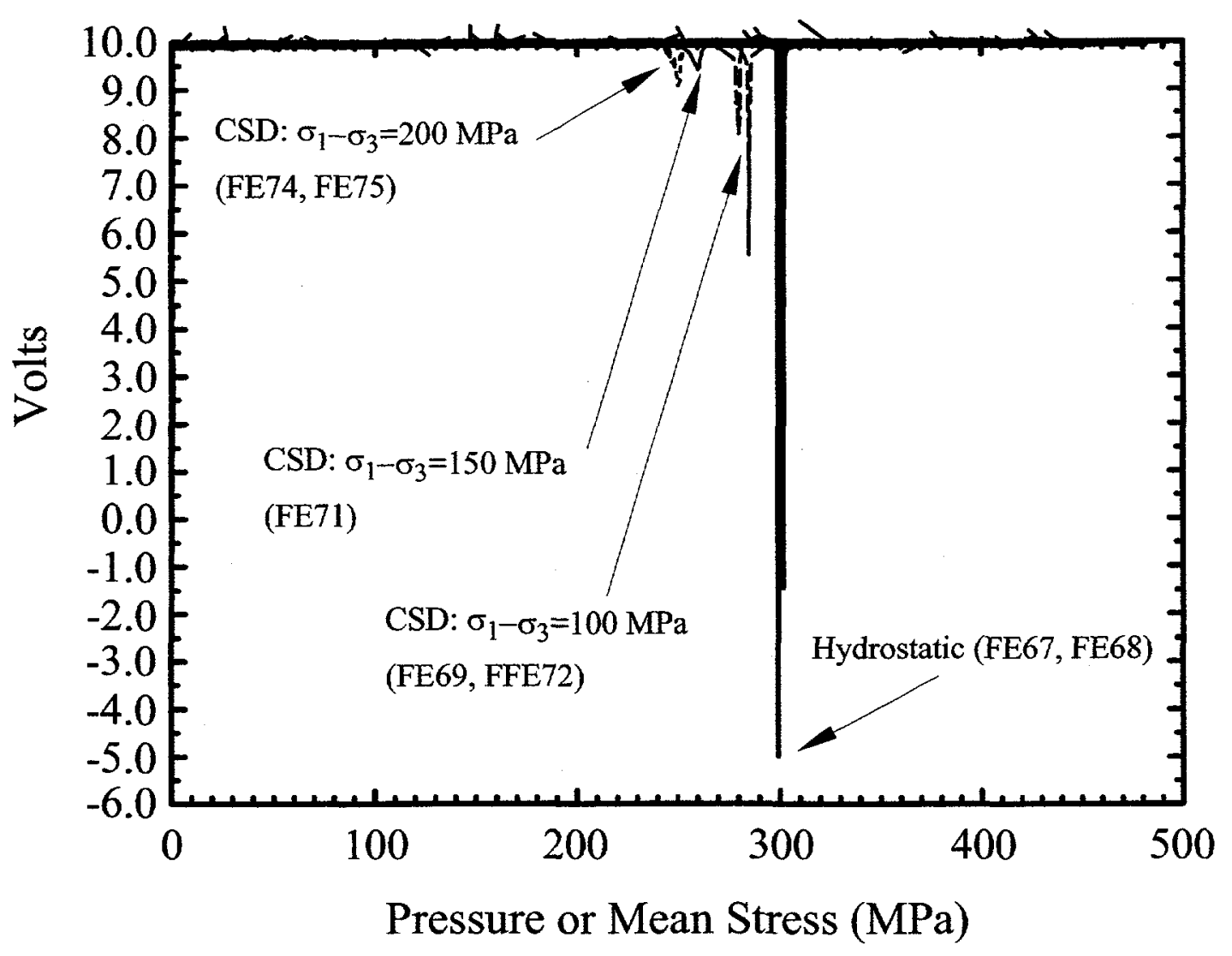

Figure 2.9: Plot of discharge voltage versus pressure or mean stress for representative hydrostatic compression and CSD experiments on biased, poled 424 ceramic, respectively. ${ }^{10,11}$ Note the depression of the mean stress for onset of transformation with increasing stress difference. Note also the broadening of the discharge voltage signatures, that indicate slowing of the rate of transformation with increasing shear stress.

terpreted, for example, as indicating that it is the normal compressive stress on planes oriented at $45^{\circ}$ to the dipolar axis that causes the transformation. This interpretation would be reasonable only if the overwhelming majority of dipoles were oriented in the same direction. Figures 2.15-2.18 do tell us something about the relationship among the applied stress, the distribution of crystallographic features, and the transformation. Only through a quantitative understanding of the orientation distribution can we relate the stress directly to some specific feature(s) of PNZT's crystallography.

If we assume that stress orientation as well as magnitude are critical for 


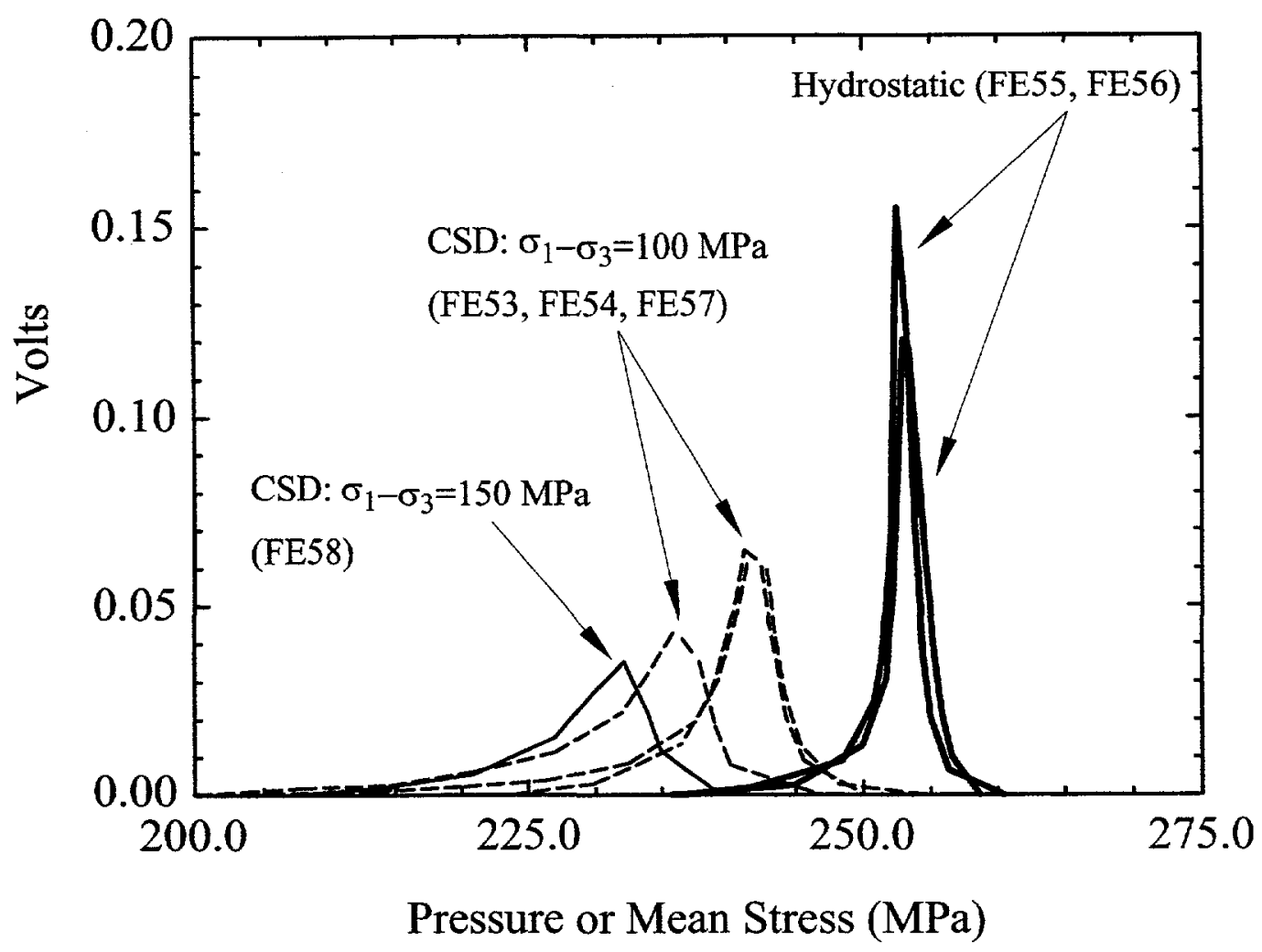

Figure 2.10: Plot of discharge voltage versus pressure or mean stress for representative hydrostatic compression and CSD experiments on unbiased, poled 541-1 ceramic, respectively. ${ }^{10,11}$ Note the depression of the mean stress for onset of transformation with increasing stress difference. Note also the broadening of the discharge voltage signatures, that indicate slowing of the rate of transformation with increasing shear stress.

onset of the transformation, then we can also explain the apparent kinetic effect of nonhydrostatic stresses. Under an applied hydrostatic pressure, every crystallographic plane experiences the same normal traction at the same instant in time, and the transformation proceeds at its maximum rate (for that temperature). Under nonhydrostatic stress, only favorably oriented crystals transform as soon as $\sigma_{1}=P_{T}^{U}$ or $P_{T}^{P}$. Progressively less-favorably oriented grains must wait until $\sigma_{1}$ increases sufficiently that the resolved normal stress 


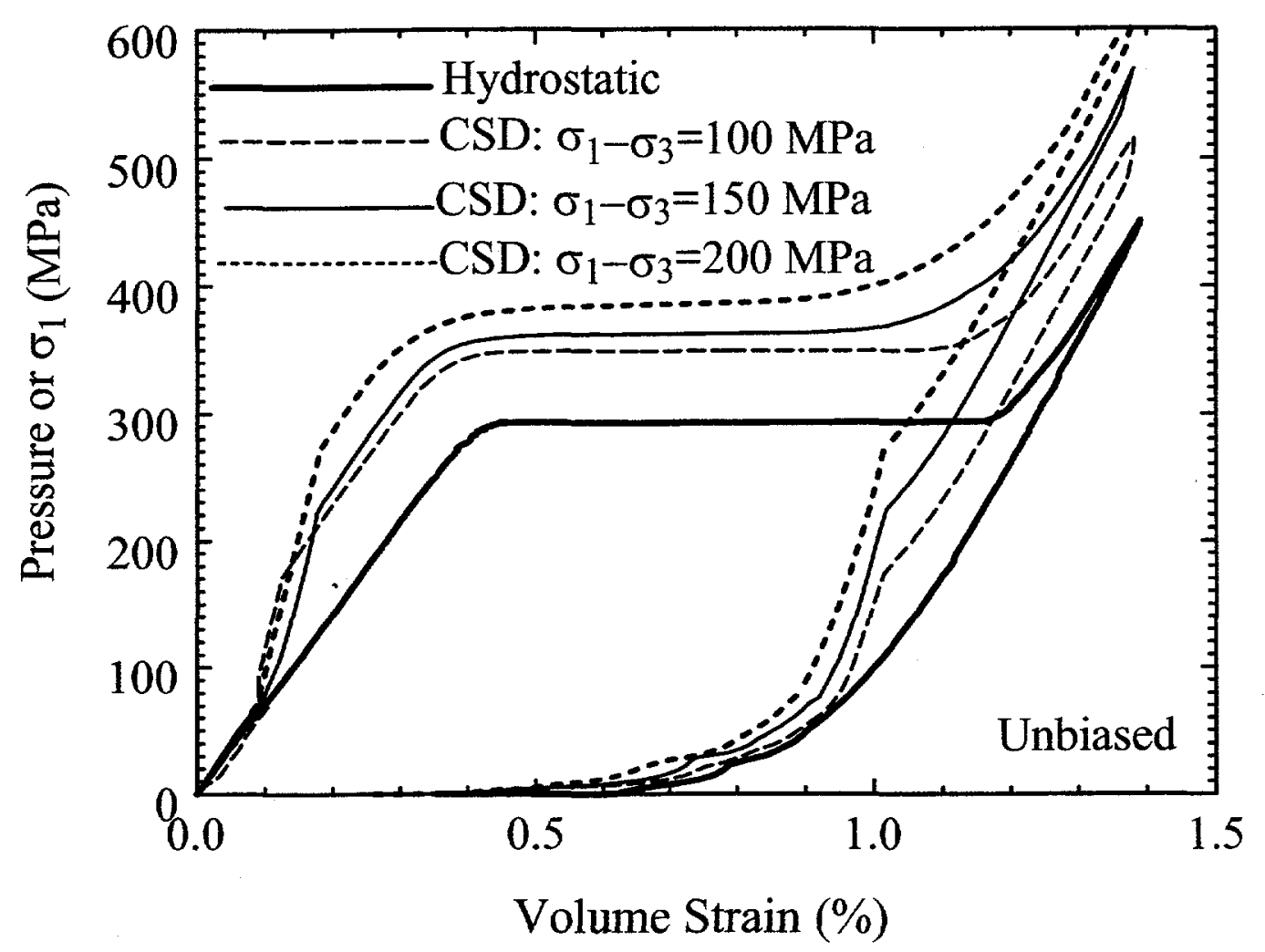

Figure 2.11: Plots of pressure or $\sigma_{1}$ versus volume strain for selected hydrostatic and CSD experiments, respectively, on unbiased, poled 424 ceramic. ${ }^{10,11}$ Sharp increases in $\sigma_{1}$ at $69 \mathrm{MPa}$ correspond to deviatoric loading of the CSD experiments. Note that at the greatest stress differences, the transformation occurs at a value of $\sigma_{1}$ that is too much higher than $P_{T}^{P}$ to be attributed to the difference to piston seal friction.

on the relevant surface(s) is equal to the critical compressive stress. Transformations that start at progressively lower confining pressures $\left(\sigma_{3}\right)$ because of higher axial stresses $\left(\sigma_{1}\right)$, necessarily take longer to reach the normal stress required for completion of the reaction, because the pressurization rate is constant in all experiments. Thus, the kinetic effect of shear stress that we have reported is real, but absolute values are related to the pressurization rate. 


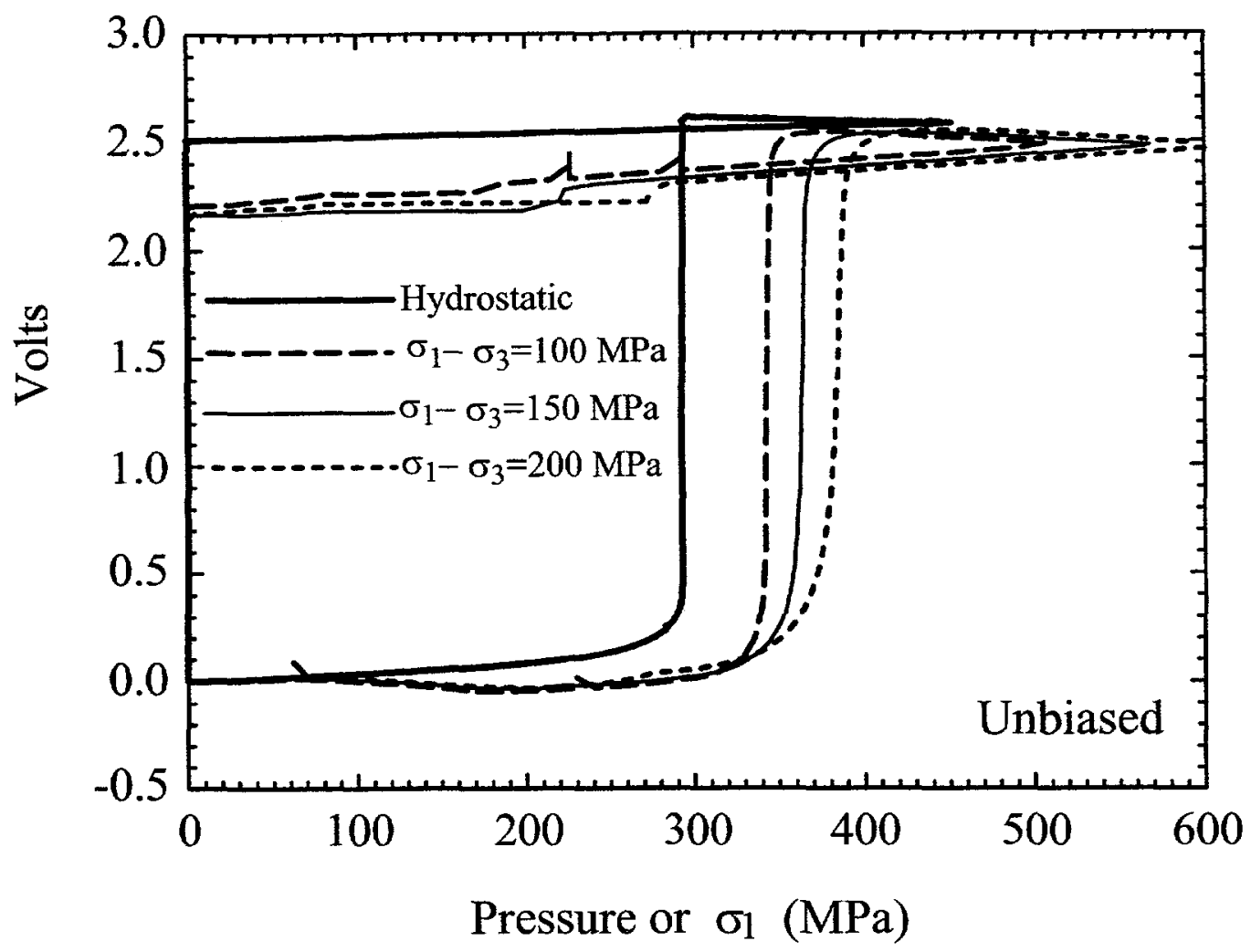

Figure 2.12: Plots of discharge voltage versus pressure or $\sigma_{1}$ for hydrostatic and CSD experiments, respectively, on unbiased, poled 424 ceramic. ${ }^{10,11}$ Note that the CSD experiments transform at values of $\sigma_{1}$ that are much higher than $P_{T}^{P}$.

Our work to date does not clearly show what normal stress is required for completion of the reaction. An additional, logical extension of our hypothesis, however is that we should expect the reaction to be complete by the time the mean stress equals $P_{T}^{U}$ or $P_{T}^{P}$, when all crystallographic surfaces experience that average normal traction under nonhydrostatic compression. Examination of Figures 2.1 and 2.7 suggest that the total volume strains accumulated in the CSD experiments on 424 ceramic are about equal to that for the hydrostatic compression experiments by the time that the mean stress equals the hydrostatic pressure for transformation. This is not quite so clear in experiments on specimens from hifire 541-1, the other ceramic on which 


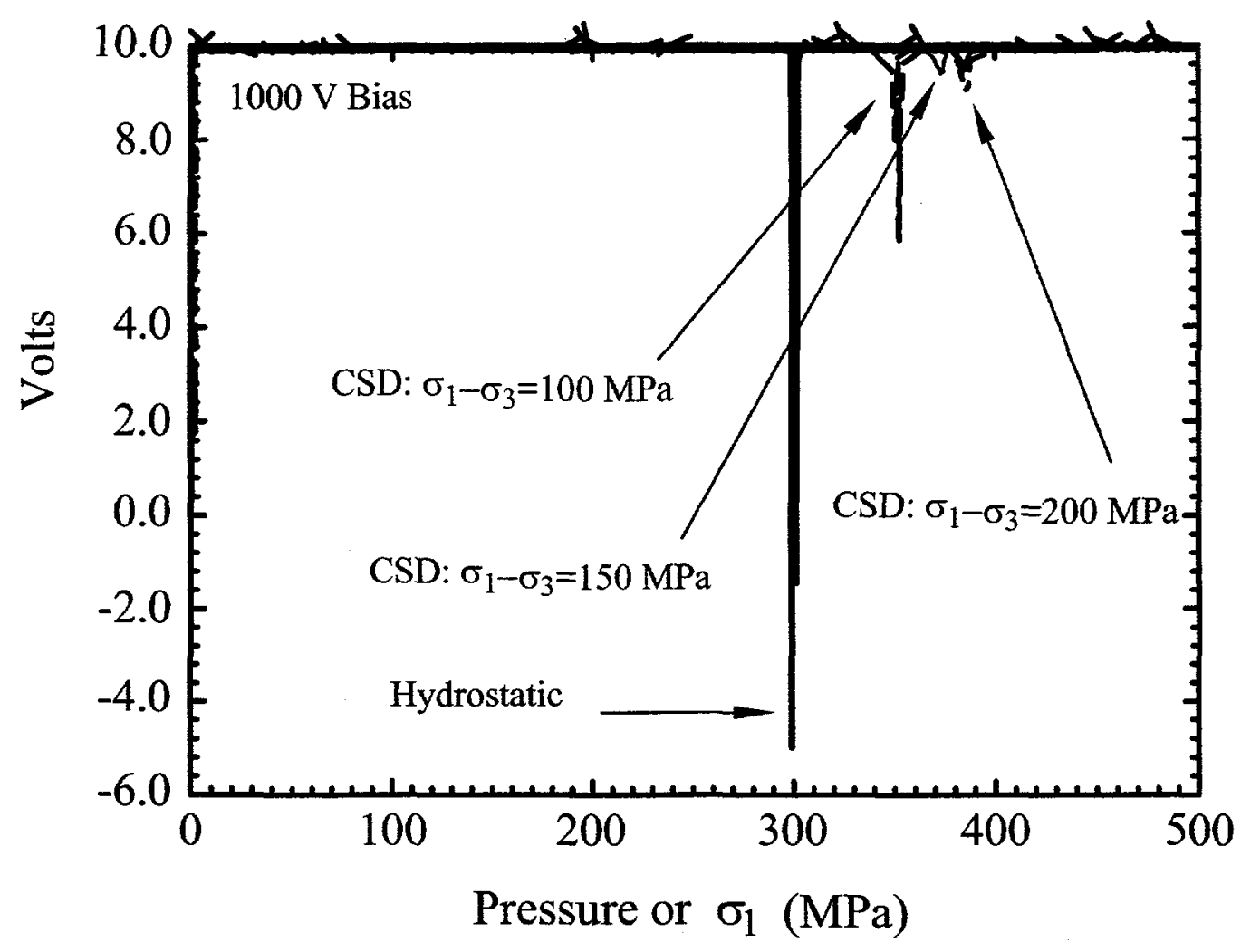

Figure 2.13: Plots of discharge voltage versus pressure or $\sigma_{1}$ for hydrostatic and CSD experiments, respectively, on biased, poled 424 ceramic. ${ }^{10,11}$ Note that the CSD experiments transform at values of $\sigma_{1}$ that are much higher than $P_{T}^{P}$. 


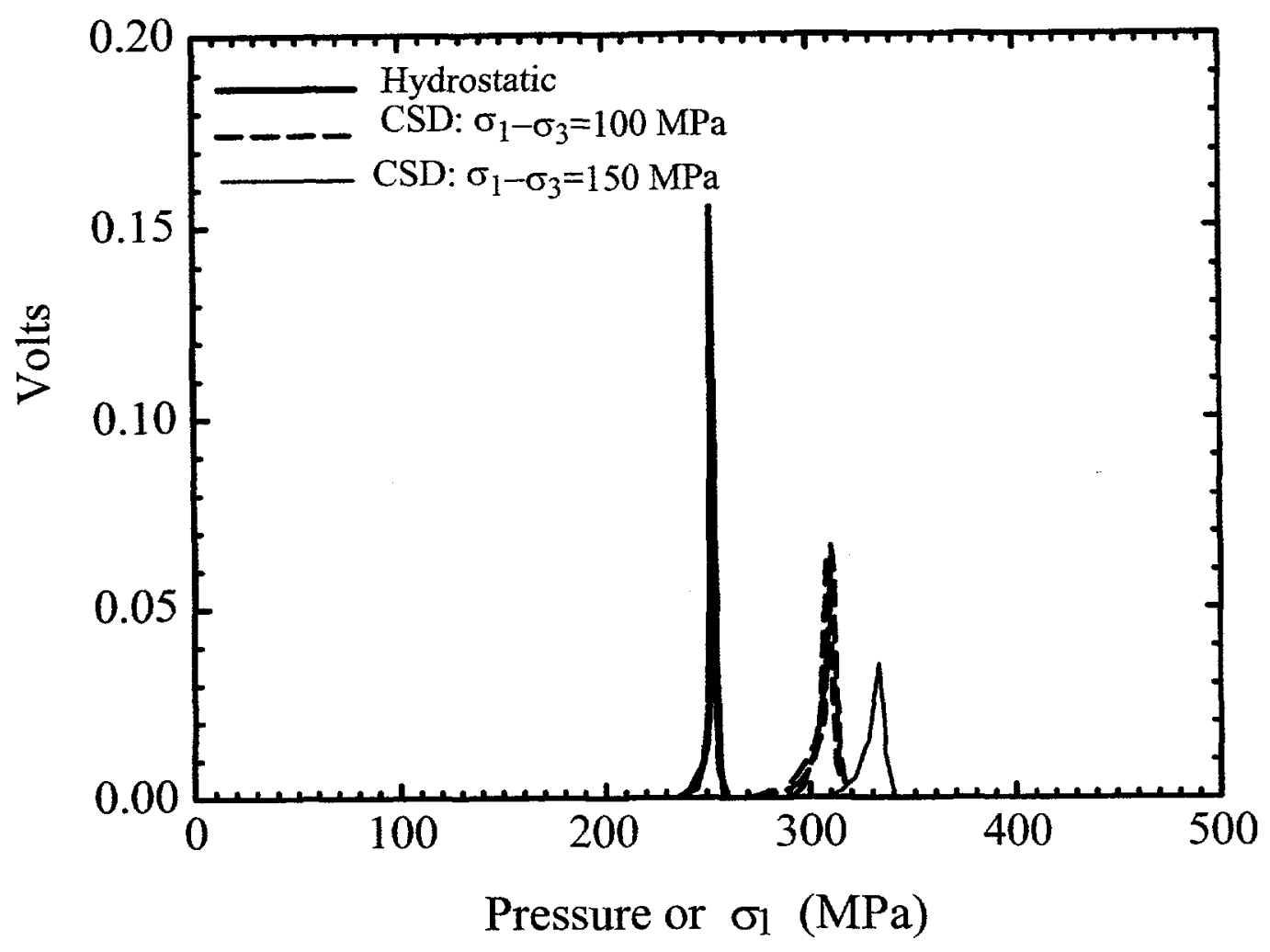

Figure 2.14: Plots of discharge voltage versus pressure or $\sigma_{1}$ for hydrostatic and CSD experiments, respectively, on unbiased, poled 541-1 ceramic. ${ }^{10,11}$ Note that the CSD experiments transform at values of $\sigma_{1}$ that are much higher than $P_{T}^{P}$. 


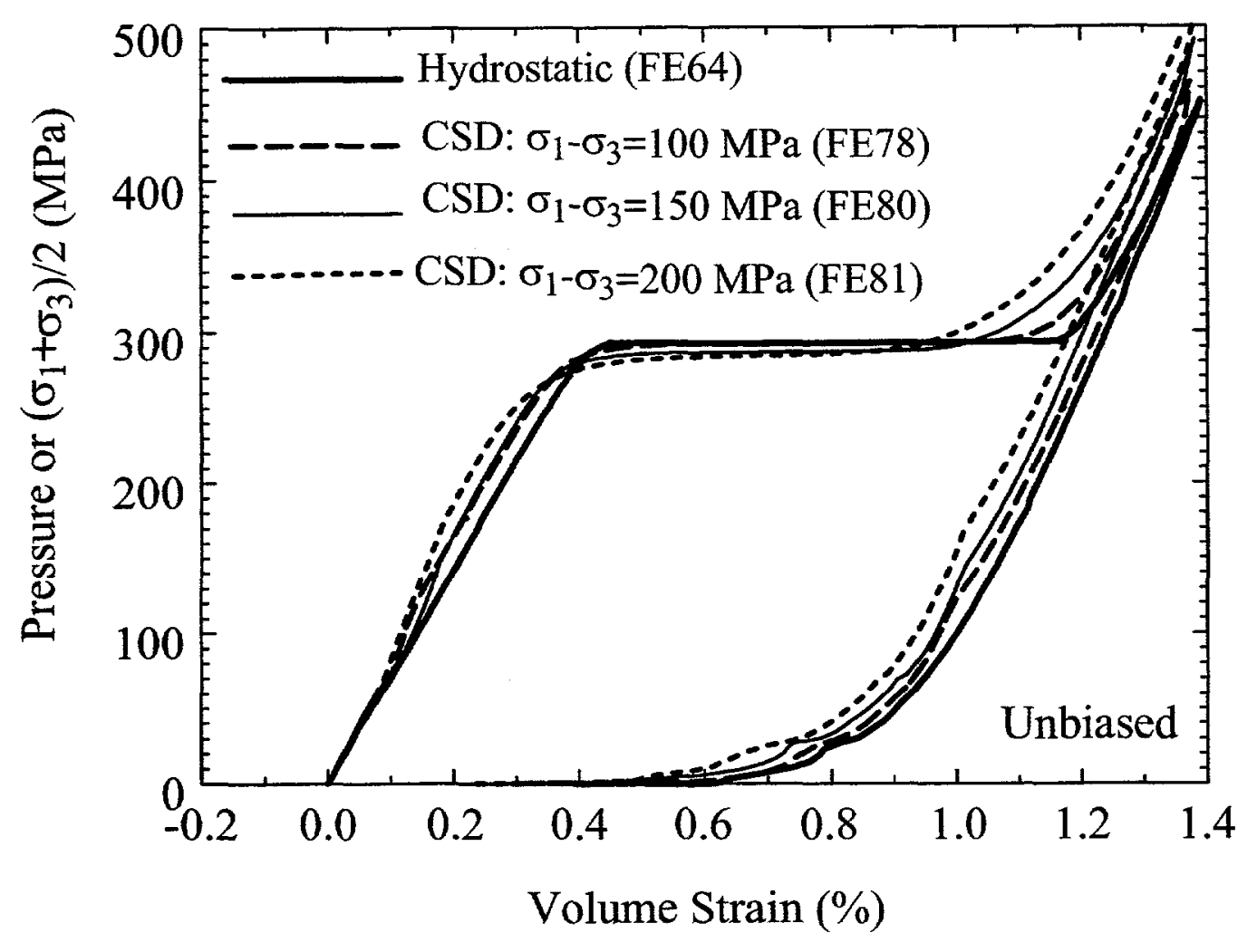

Figure 2.15: Plots of pressure or $\left(\sigma_{1}+\sigma_{3}\right) / 2$ versus volume strain for hydrostatic and CSD experiments, respectively, on unbiased, poled 424 ceramic. ${ }^{10,11}$ Note that the CSD experiments consistently transform approximately when $\left(\sigma_{1}+\sigma_{3}\right) / 2=P_{T}^{P}$. 


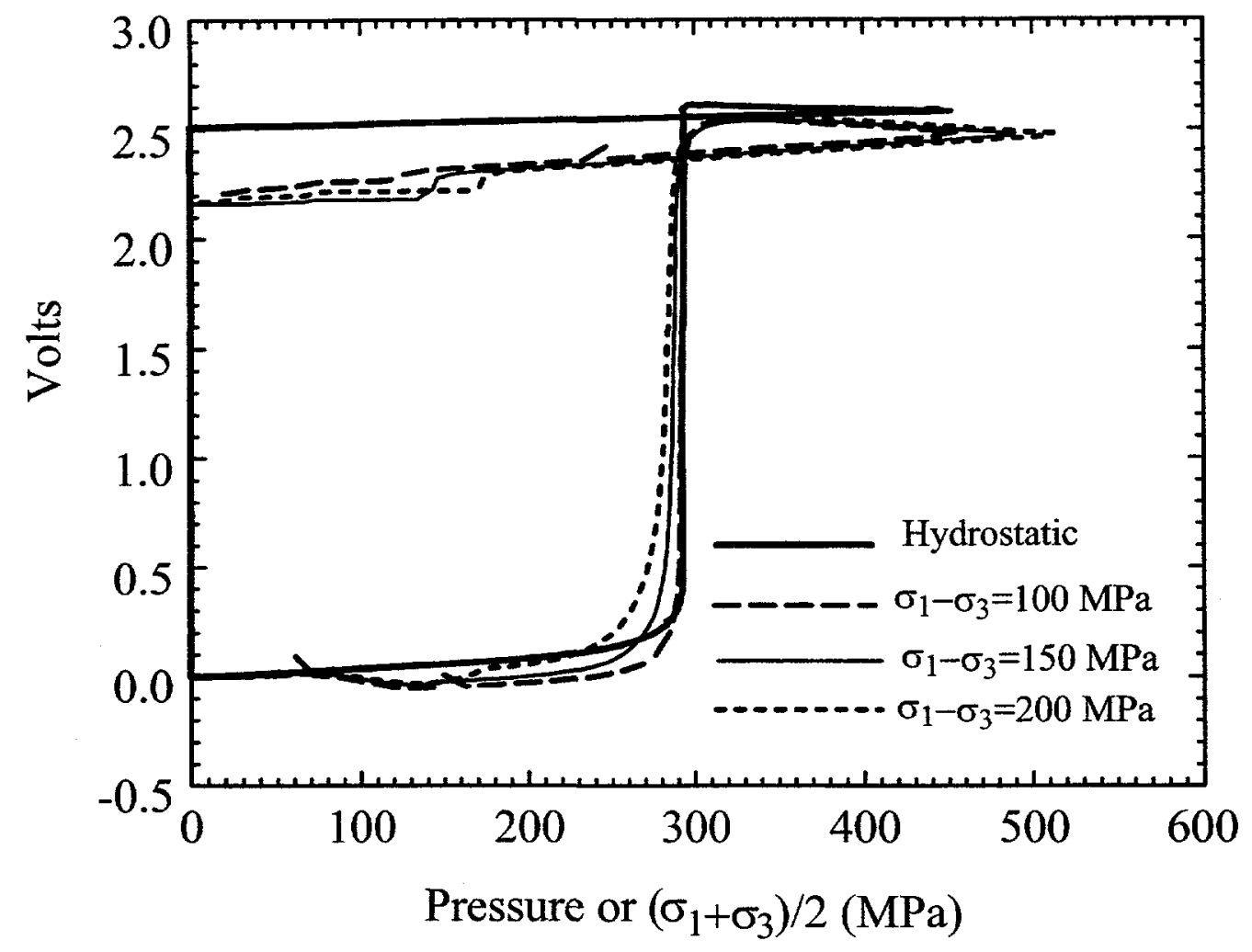

Figure 2.16: Plots of discharge voltage versus pressure or $\left(\sigma_{1}+\sigma_{3}\right) / 2$ for hydrostatic and CSD experiments, respectively, on unbiased, poled 424 ceramic. ${ }^{10,11}$ Note that the CSD experiments consistently transform approximately when $\left(\sigma_{1}+\sigma_{3}\right) / 2=P_{T}^{P}$. 


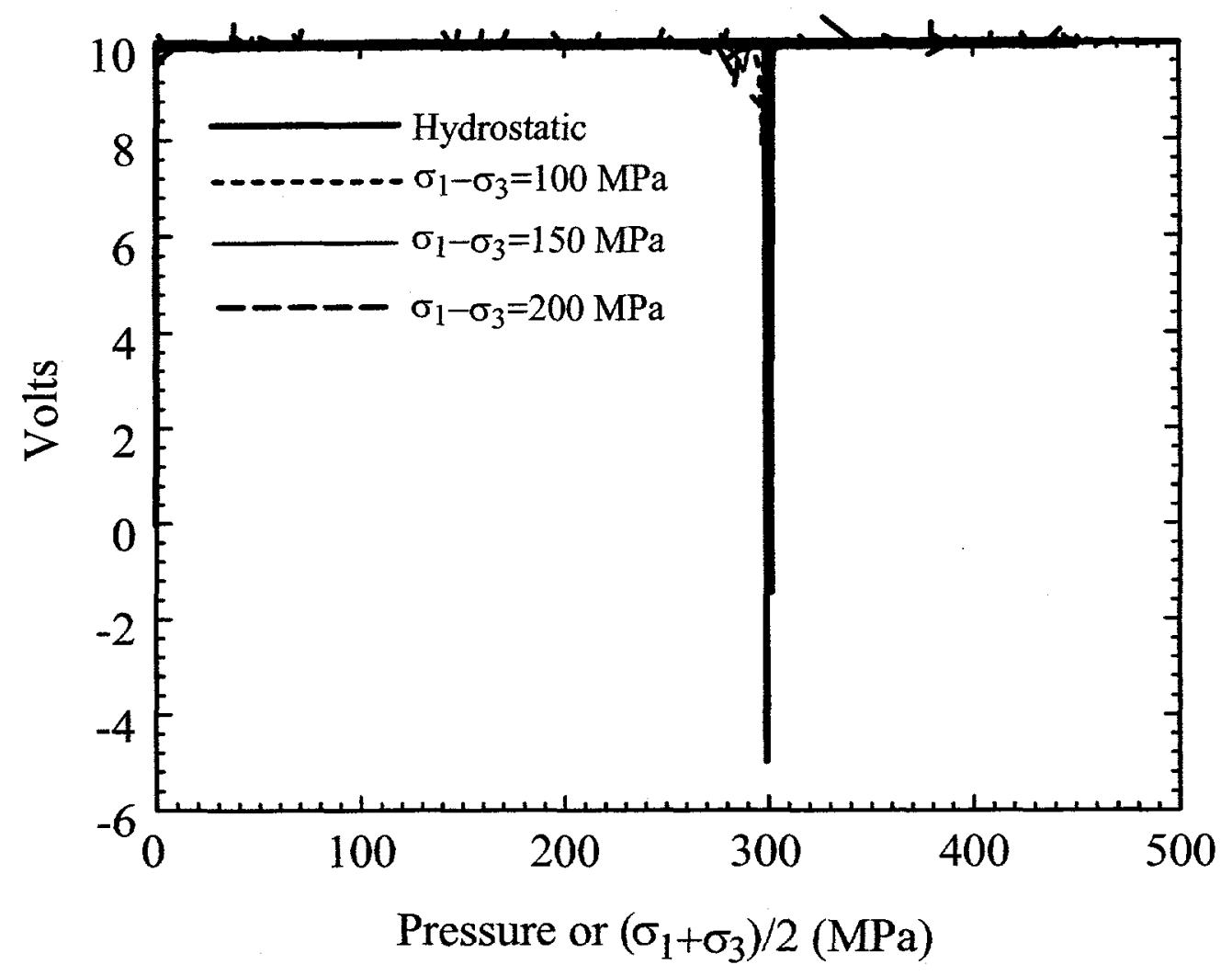

Figure 2.17: Plots of discharge voltage versus pressure or $\left(\sigma_{1}+\sigma_{3}\right) / 2$ for hydrostatic and CSD experiments, respectively, on biased, poled 424 ceramic. ${ }^{10,11}$ Note that the CSD experiments consistently transform approximately when $\left(\sigma_{1}+\sigma_{3}\right) / 2=P_{T}^{P}$. 


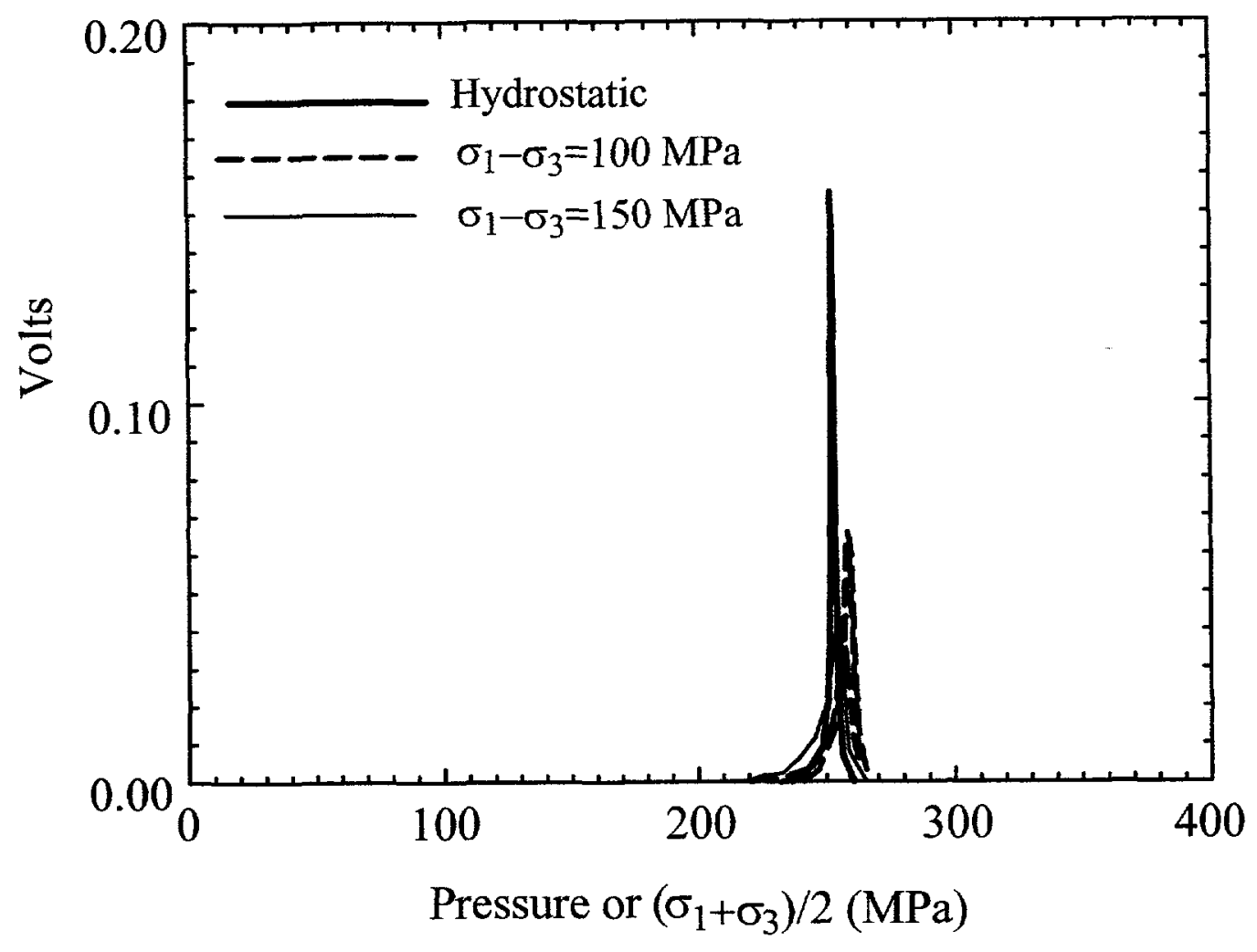

Figure 2.18: Plots of discharge voltage versus pressure or $\left(\sigma_{1}+\sigma_{3}\right) / 2$ for hydrostatic and CSD experiments, respectively, on unbiased, poled 541-1 ceramic. ${ }^{10,11}$ Note that the CSD experiments consistently transform approximately when $\left(\sigma_{1}+\sigma_{3}\right) / 2=P_{T}^{P}$. 
we have done quite a bit of testing (Figure 2.3). However, recent results from experiments on a third hifire show behavior similar to that of hifire 424 (Figure 2.19).

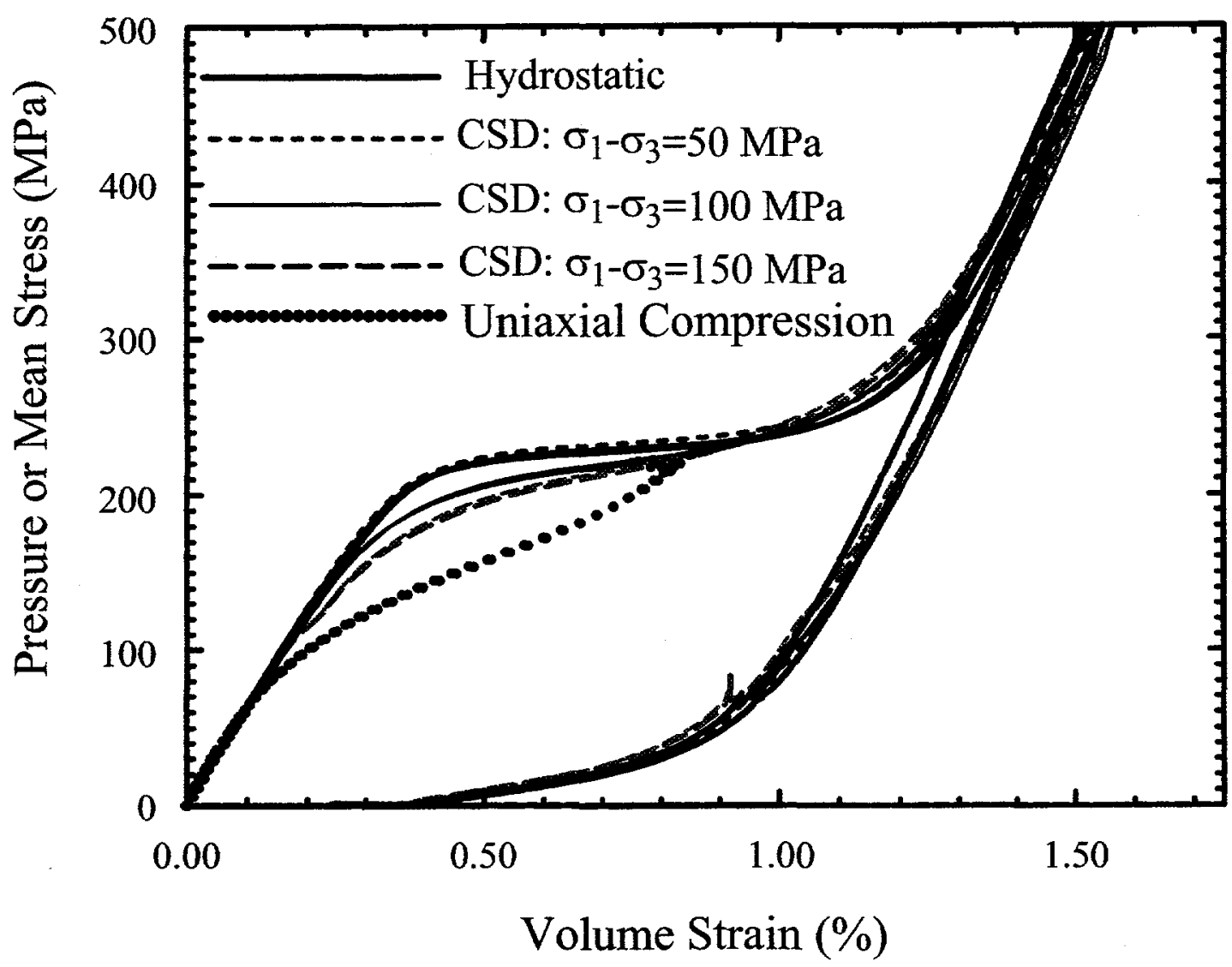

Figure 2.19: Plots of pressure or mean stress versus volume strain for hydrostatic compression, CSD and uniaxial compression experiments on unpoled specimens from a third sintering batch, hifire 453. Note that systematics are identical to those for hifires $\mathbf{4 2 4}$ and 541-1 ( $c f$. Figures 2.1 and 2.3). Like hifire 424 (Figure 2.1), transformation of 453 ceramic appears to be complete by the time that the mean stress equals $P_{T}^{U}$. Note also that unlike hifire 424, a stress difference of $50 \mathrm{MPa}$ has no detectable effect on the mean stress for transformation.

If we further assume, based on the foregoing observations, that the orientation as well as the magnitude of the compressive stress are critical for onset of the transformation, then we can make some additional predictions about 
the transformation of unpoled ceramic in uniaxial compression. Not only should the transformation begin when $\sigma_{1}=P_{T}^{U}$ as outlined above: the transformation should require a steadily increasing uniaxial compressive stress to drive the transformation forward to completion. This is because in unpoled ceramic the PNZT crystals are oriented randomly. It is assured that the appropriate crystallographic planes in at least some grains will be suitably oriented to "see" the critical stress when $\sigma_{1}=P_{T}^{U}$, and transform, as predicted above. However, steadily increasing axial stress will also be required to increase the resolved normal stress to the critical value on progressively less favorably oriented crystals. Thus, if we hold the stress constant at some point after the transformation begins, the reaction should shut off, and the volumetric strain rate should drop to zero. In principle, even in a uniaxial compression experiment, the transformation should be complete when the mean stress equals $P_{T}^{U}$. However, we might expect that some crystals are so unfavorably oriented that they never transform, at least before compressive failure intervenes.

It is less clear what predictions can be made about the behavior of poled ceramic in uniaxial and triaxial compression, because the details of the orientation distribution caused by poling are not well characterized. We will discuss some possible tests of our hypothesis using poled ceramic in the final section of this paper. In the following two sections, we will present results from the uniaxial compression experiments that we have performed on unpoled ceramic, in which we test the specific predictions outlined above. 


\section{Chapter 3}

\section{Experimental Materials and Technique}

\subsection{Experimental Materials}

The PZT $95 / 5-2 \mathrm{Nb}$ ceramic specimens used in this investigation are from the same two sintering batches used in our earlier investigations, ${ }^{7-11}$, and referred to above, hifires 424 and 541-1.

The ceramics were prepared at Sandia National Laboratories by sintering from mixed oxide powders. As stated, PNZT is used principally in shockactuated power supplies: the $\mathrm{Nb}$ dopant is added to improve its dielectric properties. In some instances, an organic pore former may also be added to increase the void space from the as-sintered value of $4-5 \%$ to a value of $7-8 \%$. Hifire 424 , had no pore former added, and has a porosity of $4-5 \%$. Hifire 541-1 had a pore former added and has a consequent porosity of $7-8 \%$. Compositions are otherwise nominally identical.

We have conducted both optical and scanning electron microscopy (SEM) on an untested specimen from hifire $424 .^{7,8}$ Optical microscopy indicates that the grain diameter of the material is approximately 5-10 micrometres. Scanning electron micrographs of chemically-etched specimens confirm the grain size estimated from optical microscopy. Owing to the unpredictability of the etching technique, the domain size is difficult to quantify with great certainty. In those areas where domains can be clearly distinguished on edge, the domain thickness is typically about 1 micrometre. Pores are often about the same diameter as the grains; the largest pores are usually irregularly shaped and cuspate, while the smallest pores are rounded and subspherical. Using SEM, we have shown in the past that pore collapse did not occur in any of our hydrostatic compression and constant stress difference experiments, though it probably occurs at higher pressures. ${ }^{7,8}$ While the presence of voids 
has probably manifested itself in the lower uniaxial compressive strength of the 541-1 ceramic compared with 424 ceramic (see below), we have seen no evidence that the presence of pores, or differences in void fractions among hifires, have otherwise influenced the results that we discuss below.

Mechanical and electrical properties of modified lead zirconate titanates $^{17}$ as well as phase equilibrium ${ }^{3}$ are very sensitive to stoichiometry and the amount of dopants. The lead oxide content, in particular, is difficult to control; hence, every hifire behaves a bit differently. ${ }^{17}$ We have now tested unpoled specimens from four different hifires, though the bulk of our experiments have been performed on material from hifire 424 , which has very consistent behavior. ${ }^{7-11}$ Both the sharp volume and capacitance drops exhibited by 424 ceramic have made identification of the onset of the transformation fairly straightforward and reproducible (Figures 2.1 and 2.2).

541-1 ceramic has shown greater intra-hifire variability of transformation pressure than 424 material, as well as several other peculiarities. ${ }^{7,8}$ We have, therefore, conducted fewer experiments on specimens from this hifire. Unlike any other PNZT hifire that $\mathrm{we}^{7-11}$ have examined, the pressure-enforced FE-AFE transformation of 541-1 ceramic is usually (though not always) completely reversible upon return to ambient pressure (Figure 2.3 ) ${ }^{7,8}$ While complete reversal has apparently also been observed by Fritz ${ }^{18, F i g .2}$ in PNZT, incomplete reversal appears to be more the norm. ${ }^{6,7,11}$ Second, dielectric response at the transformation of 541-1 ceramic is different from that which we $^{7,8}$ and others ${ }^{3}$ have typically observed at room temperature. Instead of a sharp drop in capacitance and dielectric constant at the transition (as coindicated by the volume strain anomaly), 541-1 ceramic consistently exhibits a sudden increase in capacitance, followed by a final drop (Figure 2.4). Exact onset of that increase is very difficult to pick in CSD experiments (Figure 2.4). The origin of these differences in behavior is not known.

Nevertheless, as a consequence of these differences, picking the onset of the transformation has always been more difficult for 541-1 ceramic. In the past, ${ }^{7-11}$ we have been largely concerned with the manner in which nonhydrostatic stresses affect the onset of the transformation. Thus, we have

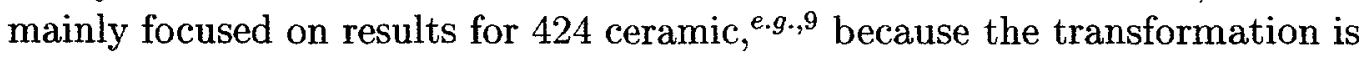
easier to identify from either volume strain or dielectric anomalies, and the results are consistent. In this paper, we are again concerned with the effects of nonhydrostatic stresses on the onset of the transformation, so we will again focus principally on results from hifire 424 . Nevertheless, despite differences in details of their behaviors, the two ceramics usually display similar trends in response to deviatoric loading. ${ }^{7,8,10,11}$ Thus, we will also present results for hifire $541-1$ in this paper. 


\subsection{Experimental Apparatus and Technique}

Save for small details, the experimental specimens, techniques and apparatus were identical to those used by us previously ${ }^{7-11}$ Test specimens were again right square prisms measuring $1.080 \mathrm{~cm} \times 1.080 \mathrm{~cm} \times 2.540 \mathrm{~cm}$, with the two opposing square faces ground flat and parallel within $0.0003 \mathrm{~cm}$. Silver electrodes were evaporated onto two opposing rectangular faces of the unpoled specimens to permit capacitance measurements if desired. ${ }^{7,8}$

Specimen strains were measured using standard, foil strain gauges bonded to the rectangular faces. In the discussion that follows, axial strains are those parallel to the long sample dimension, and lateral (or transverse) strains are those measured on the prism faces in directions perpendicular to the axial direction. Typically, three orthogonal pairs of gauges were mounted on three of the rectangular faces. Where redundant gauges (e.g., the three axial gauges) were available, they were appropriately summed and averaged before calculating the volume strain. Sign conventions for stress and strain are as outlined previously. In uniaxial compression experiments, of course, the confining pressure is effectively zero, so $\sigma_{1}-\sigma_{3} \approx \sigma_{1}$. Note that although total strains were small in all of our experiments, axial stresses were continuously corrected for changes in cross-sectional area, using data from the lateral strain gauges.

In all but three instances, hardened steel endcaps having square dimensions that matched the specimens' were glued to the ends of the samples. In the remaining three experiments, we used outsized tungsten carbide (WC) endcaps instead of the matching, hardened steel endcaps mentioned above. We have detected no effects on sample strength as a consequence of this difference in specimen end constraints. We did detect a small effect on capacitance measurements, presumably owing to differences in distortion of the electric field at the ends of the specimens, but this effect appeared to be inconsequential. Specimen assembly was completed with a thick coat of urethane that overlapped the endcaps. The urethane coating is not really necessary for uniaxial compression experiments. We normally perform this operation to prevent intrusion of confining fluid into the ceramic during our hydrostatic and triaxial compression experiments on $\mathrm{PNZT}^{7-11}$ and other pressure-sensitive materials. However, we found the urethane useful to help contain the PNZT fragments when the specimen failed catastrophically.

All experiments were done at room temperature in the same standard liquid-medium triaxial testing apparatus used previously. ${ }^{7-11}$ For our uniaxial compression experiments, however, we had no need of the $1000 \mathrm{MPa}$ pressure vessel with which the apparatus is equipped. In uniaxial compression experiments, the pressure vessel served only as added shielding from 
the explosive failure of the test specimens. Specimens were deformed using only the stiff MTS reaction frame, equipped with a servocontrolled, 1.78 MN hydraulic actuator.

Load was measured with a load cell having a maximum rating of 0.445 MN. Calibration against known standards indicates that the load cell is accurate to $\pm 480 \mathrm{~N}$. Piston seal friction effects that we discussed earlier are negligible at atmospheric pressure. Capacitance measurements were performed using a Hewlett-Packard HP4192A LF Impedance Analyzer operating at 1 $\mathrm{kHz}$. The impedance analyzer is equipped with an analog output.

As we have noticed in the past, ${ }^{7,8}$ these capacitance measurements appear to be highly sensitive to details (e.g., stray capacitance, lead length and positioning, possibly even the placement or amount of solder used on the strain gauges) about which we have little understanding or control. The reader will note (Figures 4.10 and 4.11 , below) that about half of our measurements start near $55 \mathrm{pF}$, the value that we calculate for PNZT specimens of the size that we have used. The remainder of our experiments begin at a somewhat lower value, around $40 \mathrm{pF}$. The reason for this range in values is not understood, but appears not to be related to a particular hifire or use of the $\mathrm{WC}$ endcaps. We note that despite the range in values that we observed, the behavior of the capacitance was qualitatively the same from test to test, and between hifires (Figures 4.10 and 4.11).

The signals from the strain gauges, load cell, and impedance analyzer were sent to a Keithly 500 data acquisition system and written to the hard disk on an IBM-compatible personal computer. Preliminary data reduction was accomplished during acquisition with using the program DATAVG, ${ }^{19}$ and subsequent data analysis was performed using PSIPLOT, ${ }^{T M}$ a commerciallyavailable data processing and plotting package.

The hydraulic actuator was used to move the deformation piston gently into contact with the specimen as indicated by a very slight increase in load. The piston was backed off slightly, and then advanced under microprocessor control at a constant displacement rate corresponding to an axial strain rate, $\dot{\epsilon}$, of $1 \times 10^{-4} \mathrm{~s}^{-1}$. This same strain rate was used in all of the experiments discussed in this report. In all but two of the uniaxial compression experiments, the test continued until the specimen failed (lost load bearing capability).

In the following section, we discuss our experimental results. We begin with those for the uniaxial compression experiments on unpoled ceramic from both hifires. 


\section{Chapter 4}

\section{Experimental Results}

\subsection{Compressive Strength and Elastic Prop- erties}

Typical test results for uniaxial compression experiments on 424 and 541-1 ceramic are shown in Figures 4.1 and 4.2, respectively. Apart from the differences in fracture strength, the forms of the stress-strain plots for the two hifires are very similar, in contrast to the differences between the two hifires that we have observed and remarked upon in our earlier hydrostatic compression and CSD experiments. ${ }^{7,8}$ The stress-strain curves exhibit a number of peculiarities that are related to domain switching and occurrence of the $\mathrm{FE} \rightarrow \mathrm{AFE}$ transformation; ${ }^{6}$ these will be discussed in some detail in the section immediately following. Coincidentally, but perhaps not unimportantly, the ramping rates for pressure and mean stress of the hydrostatic and constant stress difference experiments that we have performed in the past turn out to be quite similar to the mean stress ramping rates of the uniaxial compression experiments that we have performed in this report (Figure 4.3).

Results from all of the experiments on unpoled 424 and 541-1 ceramic are listed in Tables 4.1 and 4.2. Compressive strength data $\left(\sigma_{1_{f}}\right)$ are only of peripheral concern to us here, but are included for the sake of completeness. With the exception of experiment 424-04, failure can only be described as explosive, characterized by a loud noise and instant reduction of the specimens to fine rubble. The compressive strength for specimen 424-04 was anomalously low and the resulting fragments were unusually large (Table 4.1), leading us to believe that the results are spurious, owing to some hidden flaw. Where compressive strengths were not determined (N/D), the experiments were simply terminated prior to failure with the exception of experiment 42403 , for which, as noted, virtually all of the strain gauges failed prematurely 


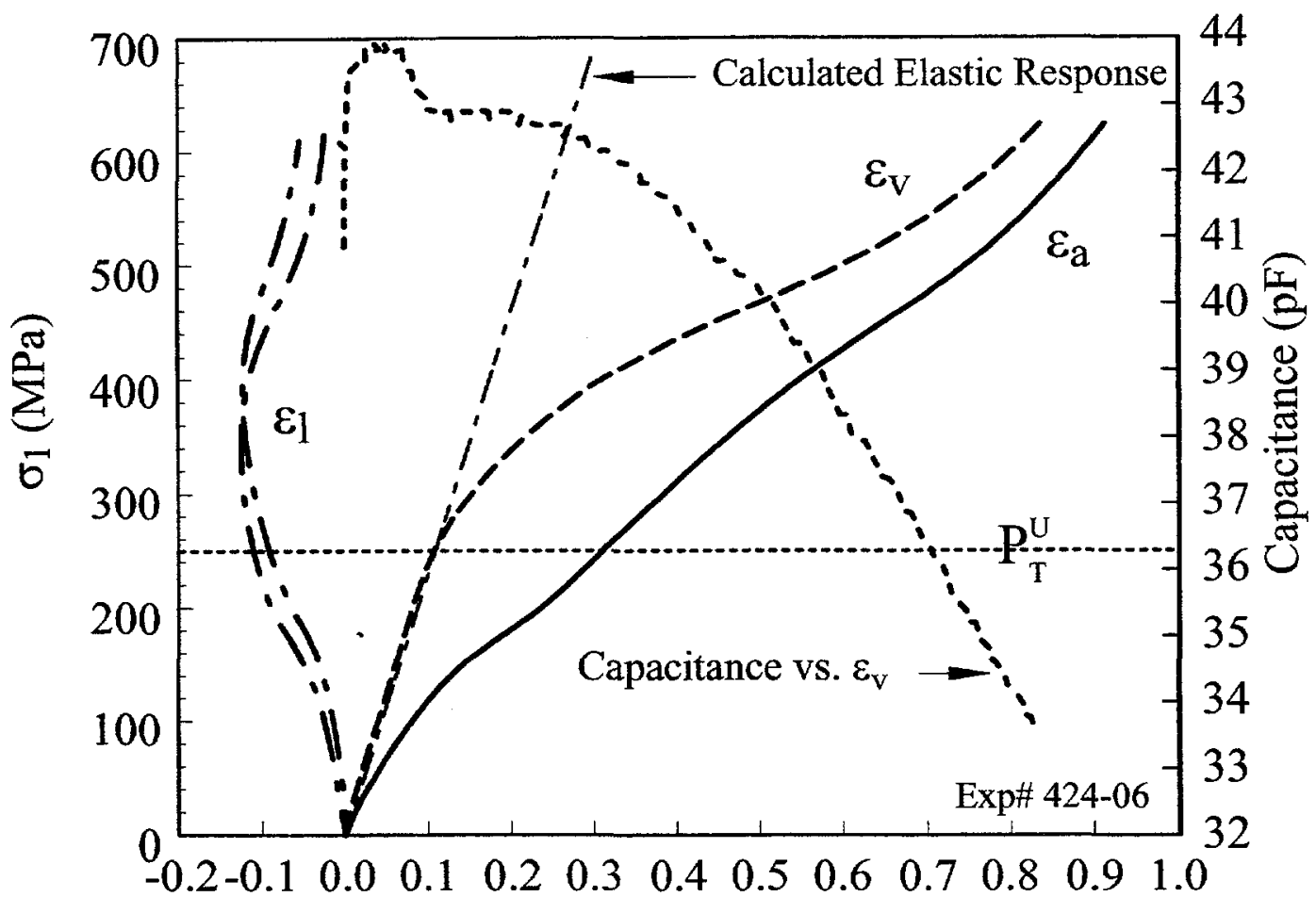

Axial, Lateral and Volume Strains (\%)

Figure 4.1: Typical uniaxial compression experiment on unpoled 424 ceramic. $\sigma_{1}$ is plotted against axial $\left(\epsilon_{a}\right)$, lateral $\left(\epsilon_{l}\right)$ and volume $\left(\epsilon_{v}\right)$ strains. Capacitance is plotted on the right hand ordinate versus $\epsilon_{v}$. Note that $\epsilon_{v}$ departs from the calculated elastic, volumetric response when $\sigma_{1}=P_{T}^{U}$. Note also that an inflection point occurs in the capacitance signature at that same volume strain and $\sigma_{1}$. This experiment was not taken to failure.

and the test was halted. Of course, where tests were terminated prior to failure, the volume strain at failure, $\epsilon_{v_{f}}$, was also not determined.

Of greater importance here, we performed a series of unload/reload cycles on the specimens used in experiments 424-01 and 541-1-01 (Figures 4.4-4.5 and 4.6-4.7, respectively). Only two cycles were performed in the former experiment, both for $\sigma_{1} \leq 200 \mathrm{MPa}$. Four cycles were performed on the latter experiment, two with $\sigma_{1} \leq 160 \mathrm{MPa}$, and two at stresses greater than 300 $\mathrm{MPa}$. At stresses below $100 \mathrm{MPa}$, the axial strains are approximately linear 


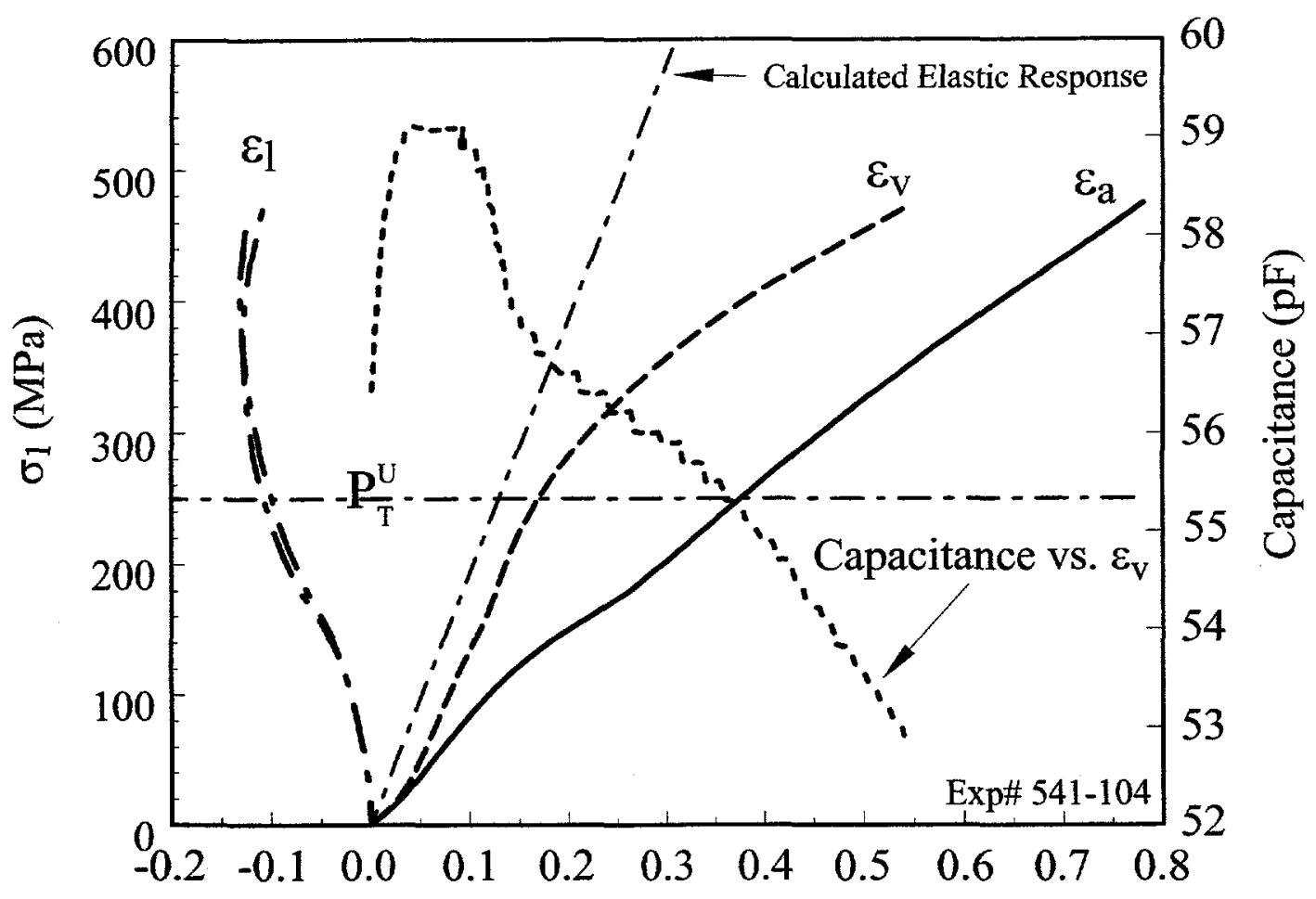

Axial, Lateral and Volume Strains (\%)

Figure 4.2: Typical uniaxial compression experiment on unpoled 541-1 ceramic. $\sigma_{1}$ is plotted against axial $\left(\epsilon_{a}\right)$, lateral $\left(\epsilon_{l}\right)$ and volume $\left(\epsilon_{v}\right)$ strains. Capacitance is plotted on the right hand ordinate versus $\epsilon_{v}$. Note that $\epsilon_{v}$ departs from the calculated elastic, volumetric response when $\sigma_{1}=P_{T}^{U}$. Note also that an inflection point occurs in the capacitance signature at that same volume strain and $\sigma_{1}$. The "toe" discussed in the text is visible as an early nonlinearity in the axial and volume strain records at stresses below 40 MPa. This experiment was not taken to failure.

and return essentially to zero upon complete unloading. At stresses above 100 $\mathrm{MPa}$, permanent small axial strains are accumulated in both experiments, which can probably be attributed to domain switching. e.g.,6 However, for all axial stresses below $200 \mathrm{MPa}$ the behavior is essentially isovolumetric (Figures 4.4 and 4.6 ) and therefore presumably unaffected by the transformation 


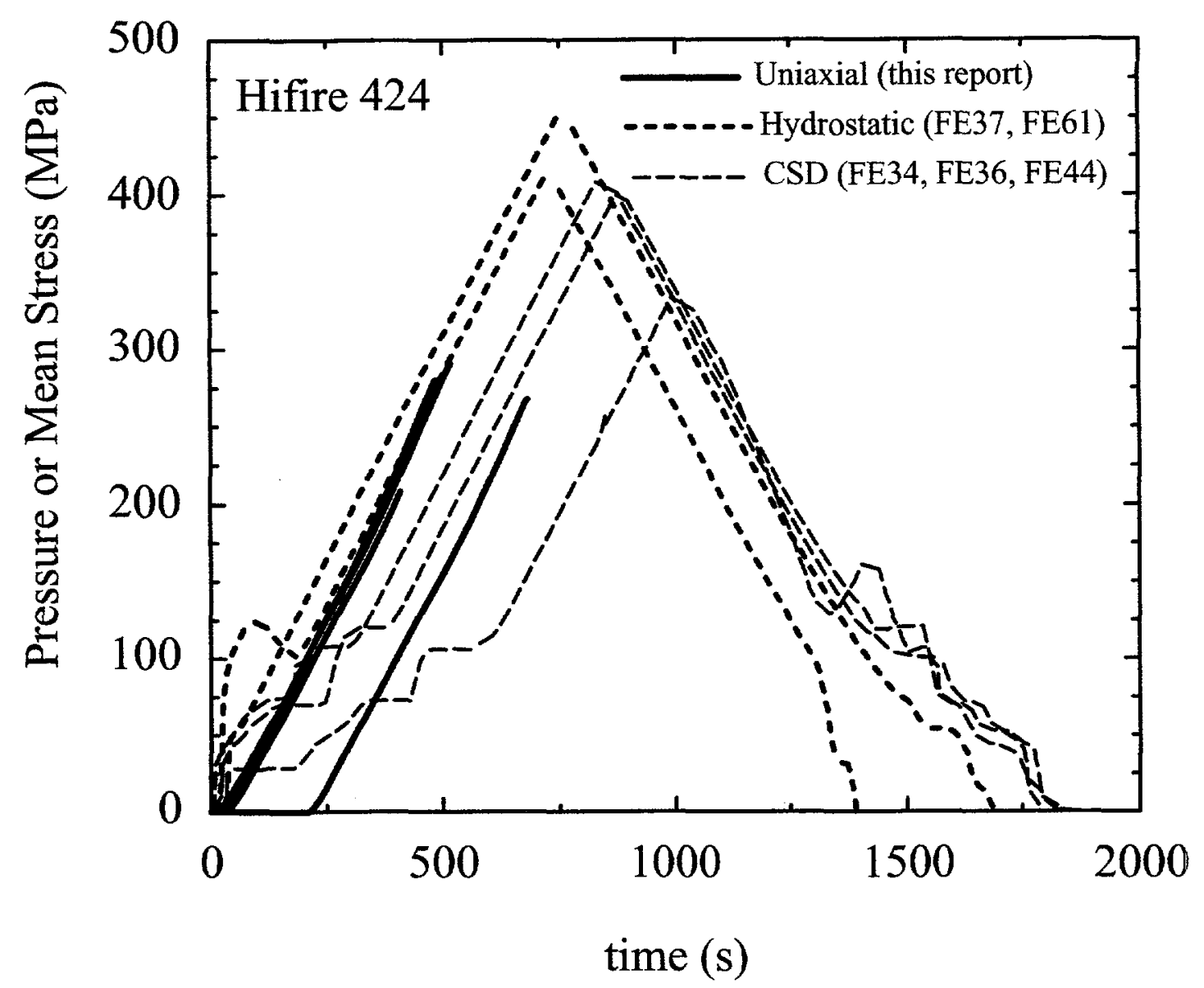

Figure 4.3: Representative pressure and mean stress ramping rates for hydrostatic compression, ${ }^{7,8}$ uniaxial compression and constant stress difference experiments on unpoled 424 ceramic. Rates are similar in all cases.

reported earlier by Fritz. ${ }^{6}$ We therefore assume that the elastic properties measured in this stress regime are representative of the pure $F_{R 1}$ phase.

At higher stresses (for experiment 541-01, at least), response becomes distinctly nonlinear and hysteretic, with increasing amounts of permanent, compressive volume strain accumulating (Figure 4.6). This is, in fact, our first, weak evidence that the phase transformation is, in fact, occurring during uniaxial compression, somewhere between 160 and $300 \mathrm{MPa}$ for hifire 541-1.

Using the unload/reload cycles for stresses below $200 \mathrm{MPa}$, we can calculate Young's modulus and Poisson's ratio for what we presume to be the pure FE phase. These values are shown in Figures 4.5 and 4.7. Averaged results 


\begin{tabular}{|c|c|c|c|}
\hline Experiment ID & $\sigma_{1_{f}}(\mathrm{MPa})$ & $\epsilon_{v_{f}}(\%)$ & Remarks \\
\hline $424-01$ & 792 & 0.92 & Multiple unload/reload cycles. \\
\hline $424-02$ & 872 & 1.05 & \\
\hline $424-03$ & N/D & N/D & All strain gauges failed, possibly de-bonded. \\
\hline $424-04$ & 479 & -3.1 & Unusually large fragments remained. \\
\hline $424-05$ & 803 & 0.96 & Capacitance measured. \\
\hline $424-06$ & $\mathrm{~N} / \mathrm{D}$ & $\mathrm{N} / \mathrm{D}$ & Test terminated prior to failure; capacitance measured. \\
\hline $424-07$ & 839 & 0.94 & Capacitance measured; WC endcap used. \\
\hline $424-10$ & 881 & 0.91 & Deformation interrupted three times. WC endcap used. \\
\hline $424-11$ & 799 & 0.88 & Deformation interrupted three times. WC endcap used. \\
\hline $424-12$ & 766 & 0.96 & Deformation interrupted three times. WC endcap used. \\
\hline
\end{tabular}

Table 4.1: Uniaxial compression experiments on unpoled hifire 424 ceramic. $\sigma_{1_{f}}$ and $\epsilon_{v_{f}}$ are the compressive stress and volume strain at failure, respectively. Strain rate was $1.0 \times 10^{-4} \mathrm{~s}^{-1}$.

\begin{tabular}{|c|c|c|c|}
\hline Experiment ID & $\sigma_{1_{f}}(\mathrm{MPa})$ & $\epsilon_{v_{f}}(\%)$ & Remarks \\
\hline $541-1-01$ & 648 & 0.81 & Multiple unload/reload cycles. \\
\hline $541-1-02$ & 651 & 0.85 & \\
\hline $541-1-03$ & 708 & 0.90 & Noise on load cell; capacitance measured. \\
\hline $541-1-04$ & N/D & N/D & Test terminated prior to failure; capacitance measured. \\
\hline $541-1-05$ & 638 & 0.81 & Capacitance measured; WC endcap used. \\
\hline
\end{tabular}

Table 4.2: Uniaxial compression experiments on unpoled, hifire 541-1 ceramic. $\sigma_{1_{f}}$ and $\epsilon_{v_{f}}$ are the compressive stress and volume strain at failure, respectively. Strain rate was $1.0 \times 10^{-4} \mathrm{~s}^{-1}$.

for $\mathrm{E}$ and $\nu$ for hifires 424 and 541-1 are 119,500 $\mathrm{MPa}$ and 0.23 , and 104,600 $\mathrm{MPa}$ and 0.23 , respectively. We can calculate the anticipated elastic volumetric response to uniaxial compressive loading from the averaged results for $\mathrm{E}$ and $\nu$ for each hifire. These results will be of use to us to determine if and when the transformation begins in our uniaxial compression experiments.

We remark in passing that although we have measured the elastic properties of only one specimen from each hifire, we have reasonable confidence in these results. Assuming mechanical isotropy, we can calculate the the bulk modulus, $\mathrm{K}$, from $\mathrm{E}$ and $\nu$, obtaining values of $73,765 \mathrm{MPa}$ and and 64,568 MPa for 424 and 541-1 ceramic, respectively. Using these values, we can calculate the expected response of these two hifires to hydrostatic compression. The results compare very favorably with many such direct measurements performed earlier on 424 and 541-1 ceramic (e.g., Figures 4.10 and 4.11, below). 


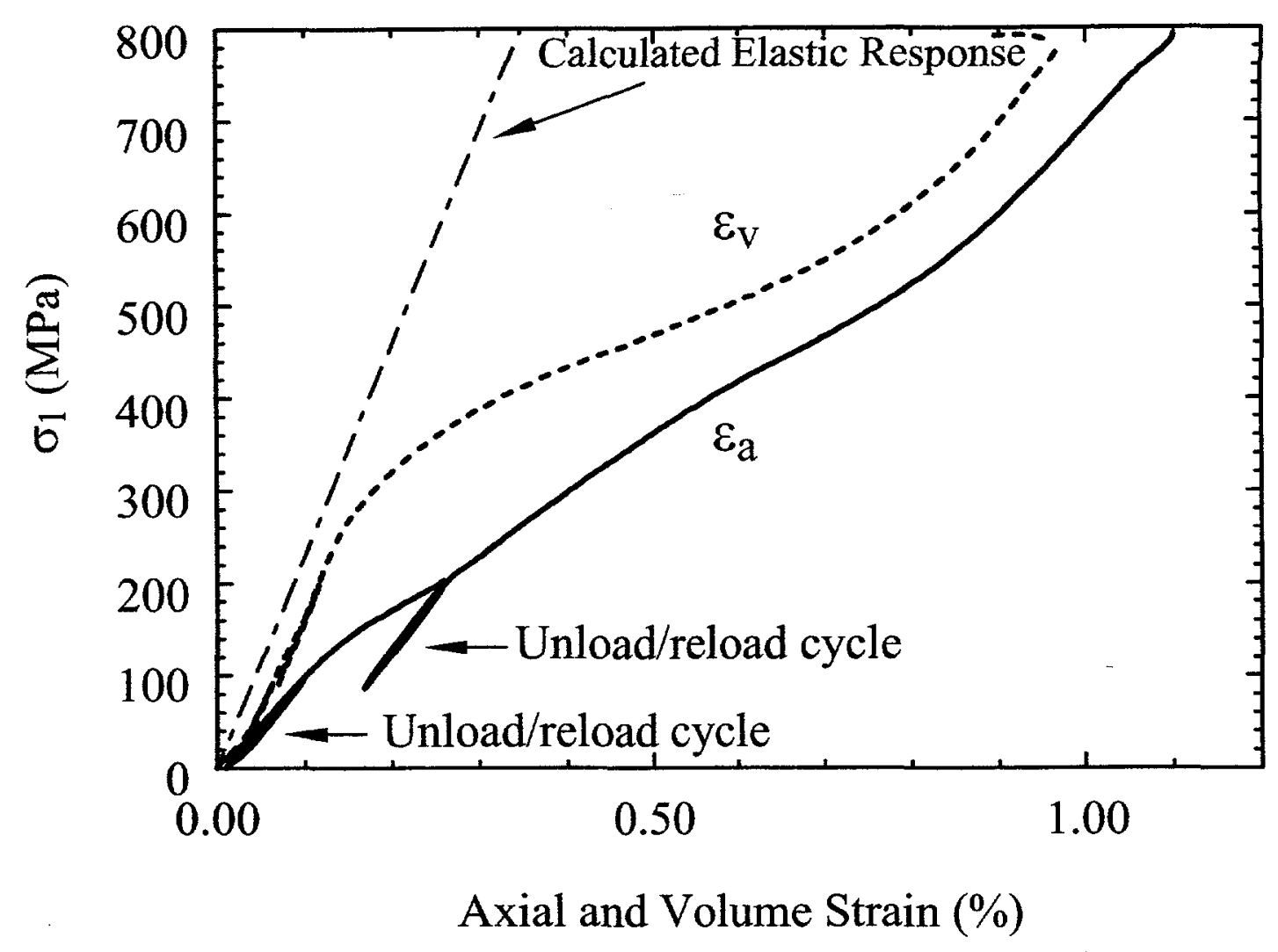

Figure 4.4: Unload/reload cycles used to measure Young's modulus (E) and Poisson's ratio $(\nu)$ in early stages of a uniaxial compression experiment on 424 ceramic. Although permanent axial strains begin to accumulate at stresses around $200 \mathrm{MPa}$, volume strains remain elastic. Calculated volumetric elastic response is shown for reference.

\subsection{Mechanical Behavior and Evidence for Oc- currence of the Phase Transformation}

Given the explosive nature of failure and the obviously brittle character of the two ceramics, the behavior of all measured and calculated strains is quite remarkable. The lateral strains observed in experiments taken to failure completely reverse twice (Figures 4.8 and 4.9). Axial and volumetric strains are large, nonlinear, and compressive right up to failure. Had not Fritz ${ }^{6}$ reported similar results for uniaxial compression experiments on PNZT, we would have questioned our experimental technique. From data very similar 


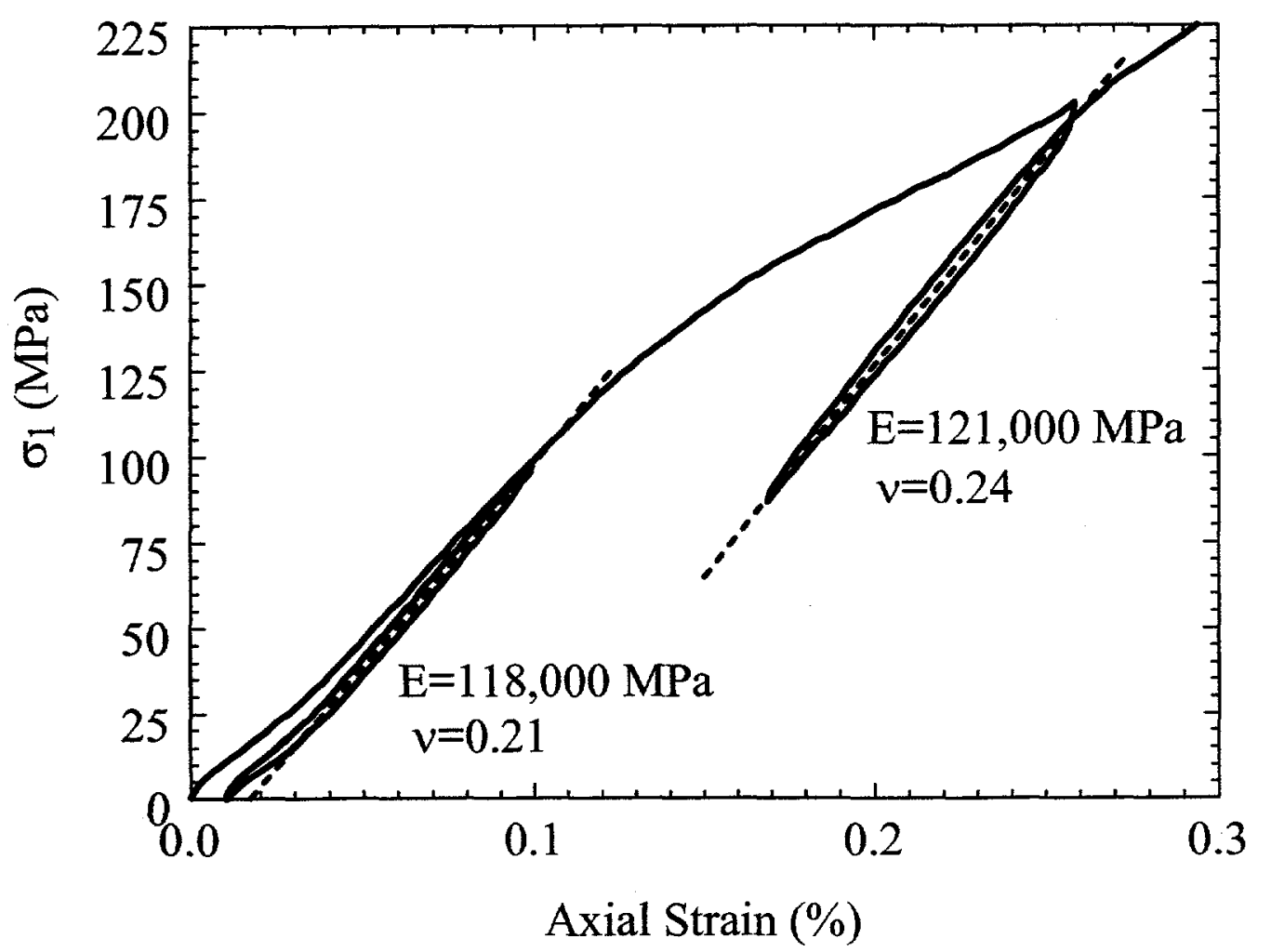

Figure 4.5: Detail of Figure 4.4.

to these, Fritz ${ }^{6}$ concluded that the $\mathrm{FE} \rightarrow \mathrm{AFE}$ transformation occured in his uniaxial compression experiments.

Fritz identified the transformation largely by comparing stress-strain data similar to those shown in Figures 4.1 and 4.2 with the expected elastic response of the pure FE phase. The expected elastic response was estimated from acoustic velocity measurements performed on specimens during uniaxial compression. Departures from the theoretical elastic response were related to domain switching and/or occurrence of the $\mathrm{FE} \rightarrow \mathrm{AFE}$ transformation. From his experiments, Fritz ${ }^{6}$ concluded that following a brief period of isovolumetric, but nonlinear axial strain response in the stress range of 0-50 MPa caused by domain realignment alone, the FE-AFE phase transformation was subsequently triggered by the uniaxial compressive stress, beginning at about 50 $\mathrm{MPa}$ and still continuing at his reported compressive failure stress of about 


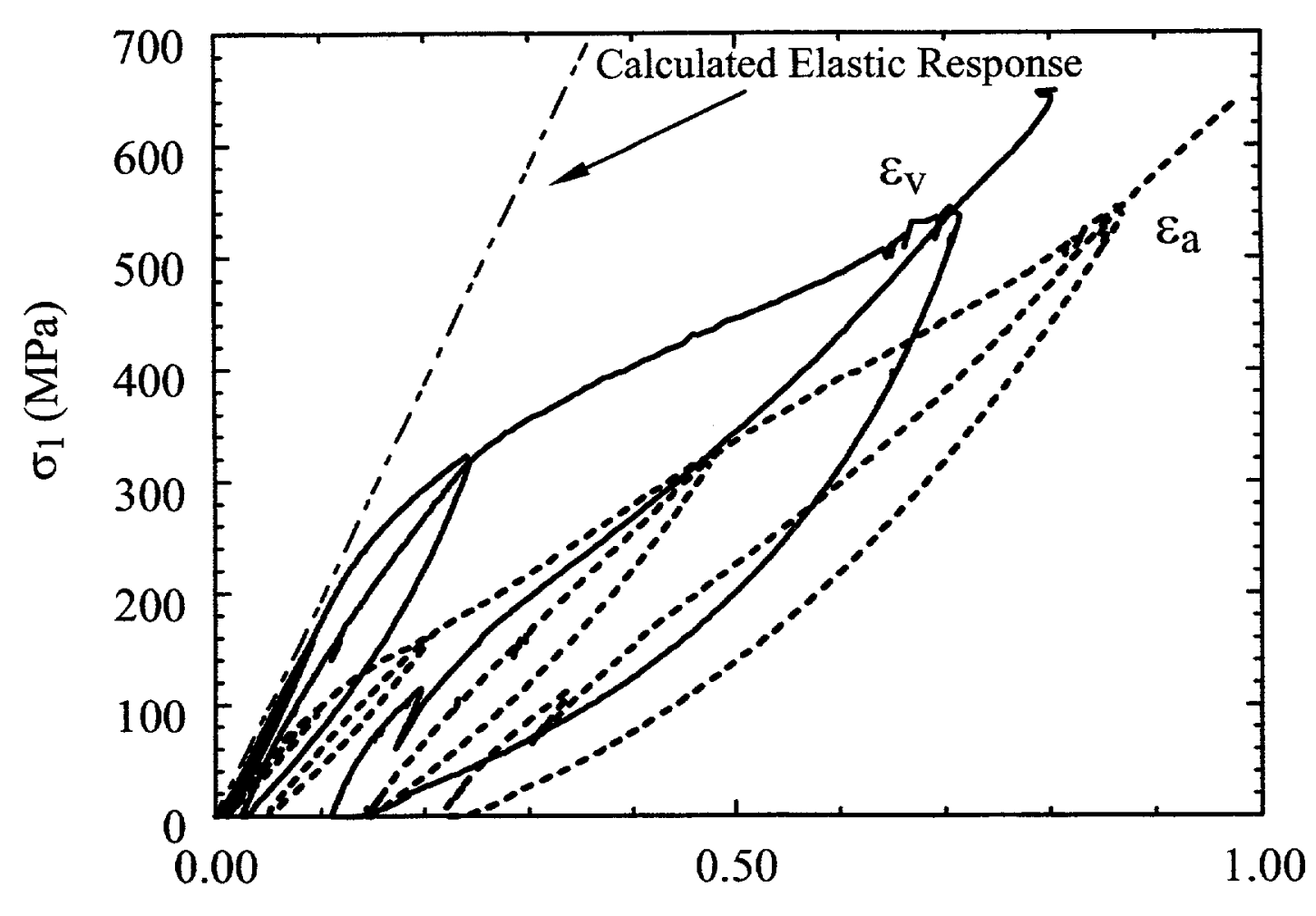

Axial and Volume Strain (\%)

Figure 4.6: Unload/reload cycles used to measure Young's modulus (E) and Poisson's ratio $(\nu)$ at various stages of a uniaxial compression experiment on 541-1 ceramic. Volume strains are essentially elastic for stresses below $160 \mathrm{MPa}$, with some permanent strains accumulating at higher stresses. Permanent axial strains begin to accumulate at stresses somewhere above $160 \mathrm{MPa}$. Calculated volumetric elastic response is shown for reference.

$600 \mathrm{MPa}$.

We have taken a similar approach, but absent acoustic velocity data, we have compared the expected elastic volumetric response with the observed behavior in order to identify the phase transformation. We attempted to supplement this approach with capacitance measurements (Tables 4.1 and 4.2), which have proven to be a useful diagnostic in the past. However, for reasons that are not clear, the capacitance data are much less useful for our uniaxial compression experiments than for our earlier hydrostatic compression and CSD experiments. Nevertheless, we reach conclusions that 


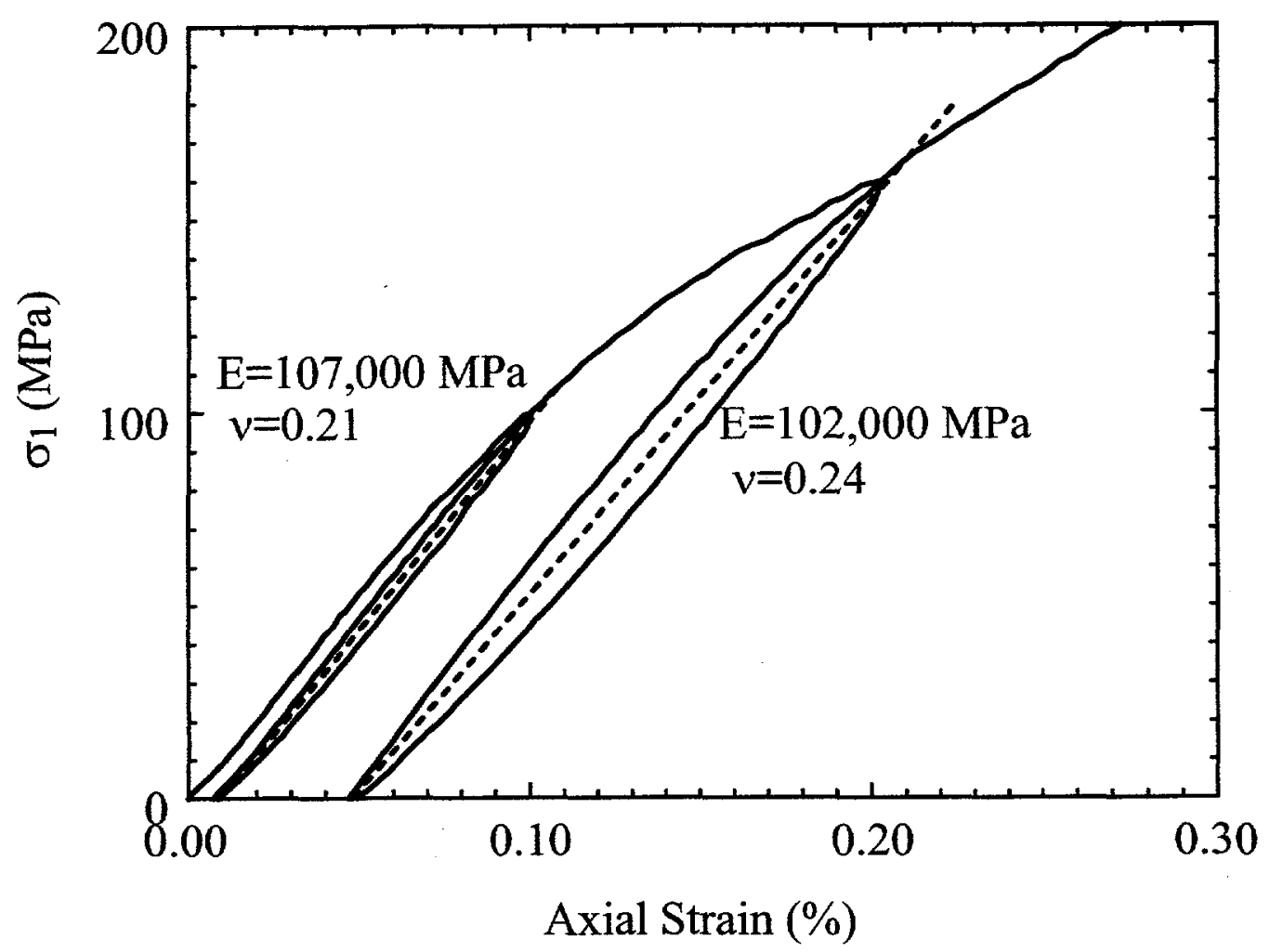

Figure 4.7: Detail of Figure 4.6 showing the two loops used to calculate $\mathbf{E}$ and $\nu$.

are similar to those of Fritz ${ }^{6}$, if somewhat simpler.

Although we frequently observe a brief episode of nonlinear stiffening (sometimes referred to as a "toe") in the $\sigma_{1}$-volume strain data for both hifires immediately upon loading (e.g., Figure 4.2), behavior is essentially linear up to stresses somewhere between 200 and $300 \mathrm{MPa}$ and strains of about $0.1-0.15 \%$ (Figures 4.1 and 4.2). It is not known what causes this early, nonlinear toe, but it could reasonably be ascribed to closing of minute cracks or grain boundary openings during loading. It might also be explained by some localized FE-AFE transformation caused by stress concentrations as a result of slightly eccentric loading, but we have no clear evidence to confirm this. It cannot be attributed to stress-induced domain switching, because that should be an isovolumetric process. ${ }^{6}$

In Figures 4.1 and 4.2, we have also plotted the calculated elastic volu- 
Capacitance $(\mathrm{pF})$

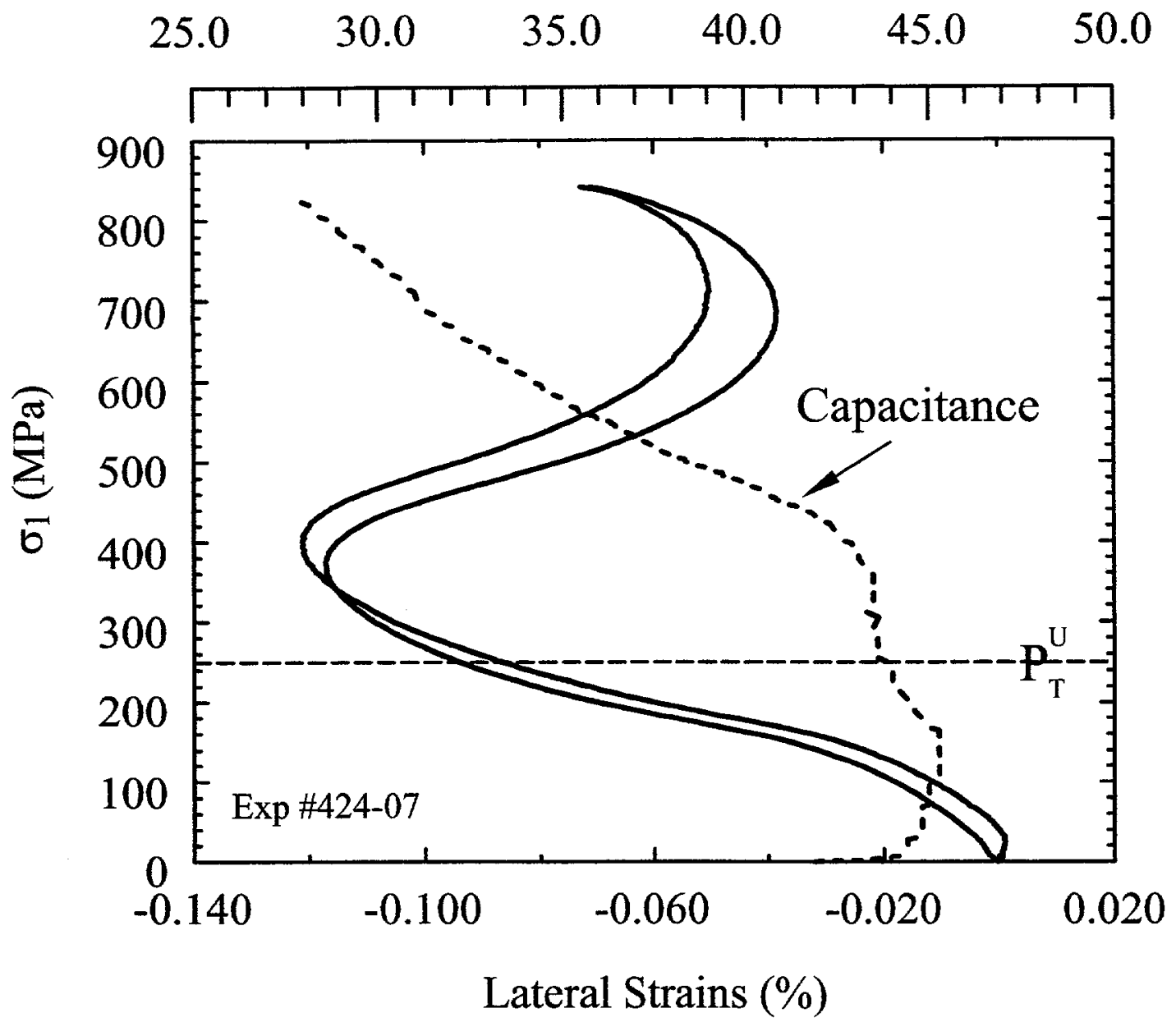

Figure 4.8: Enlarged plot of lateral strain behavior during uniaxial compression of 424 ceramic. Note the two complete reversals of the lateral strains. Early nonlinearity is probably caused by dipole rotation. Onset of reversal at about $250 \mathrm{MPa}$ probably corresponds to start of the transformation. Second reversal probably correponds to exhaustion of the transformation and/or onset of microcracking. Capacitance data are plotted against $\sigma_{1}$ using the upper abscissa.

metric strains that we would expect to be associated with uniaxial loading of the pure FE phase. Although the calculated volumetric strains are not always coincident with the measured ones in this nominally linear regime ow- 
Capacitance $(\mathrm{pF})$

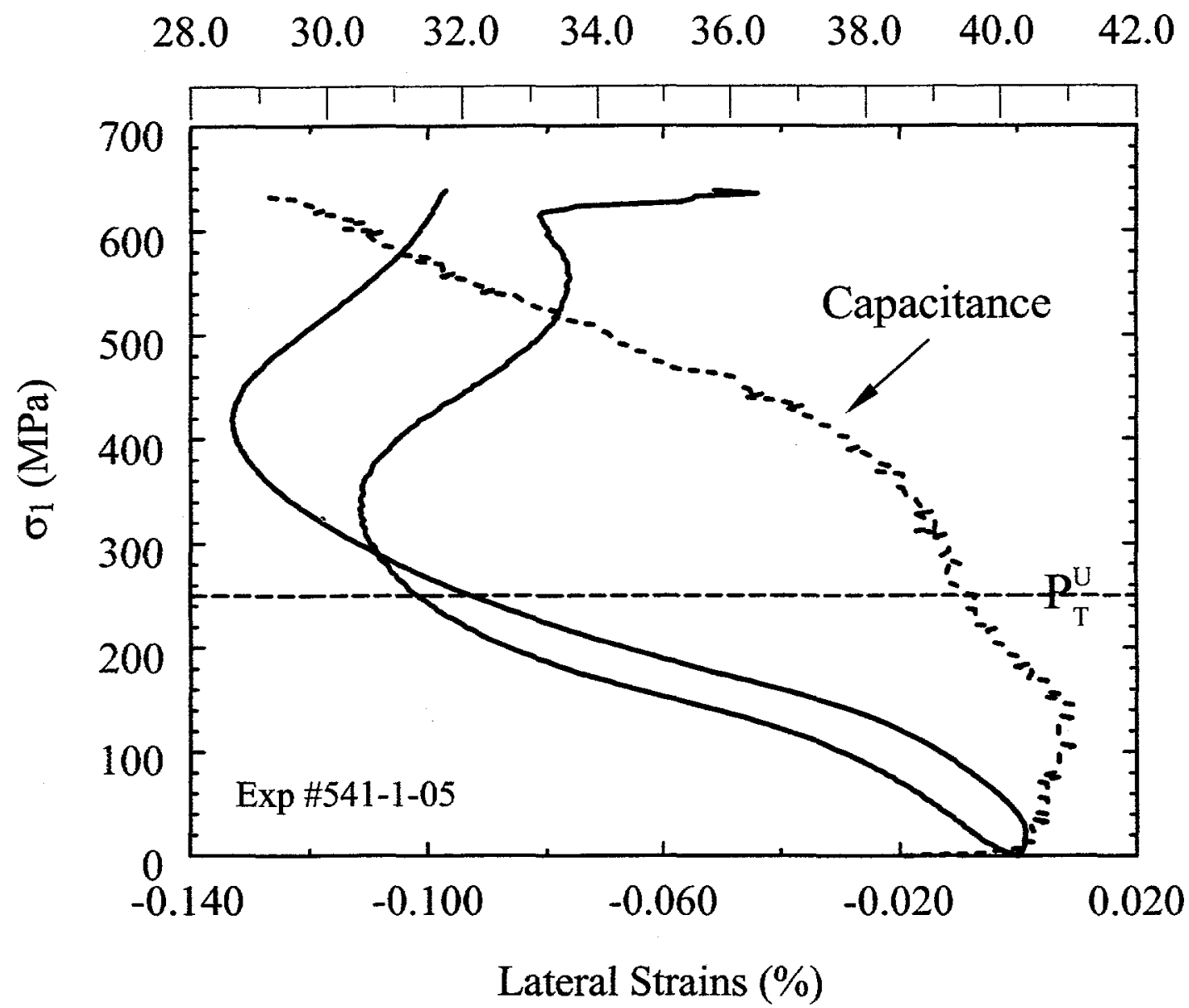

Figure 4.9: Enlarged plot of lateral strain behavior during uniaxial compression of 541-1 ceramic. Note the two complete reversals of the lateral strains. Early nonlinearity is probably caused by dipole rotation. Onset of reversal at about $250 \mathrm{MPa}$ probably corresponds to start of the transformation. Second reversal probably correponds to exhaustion of the transformation and/or onset of microcracking. Capacitance data are plotted against $\sigma_{1}$ using the upper abscissa.

ing to the toe, the slopes are always very similar. For this reason, we think that this initial, nearly linear response is simply the normal, elastic volume strain of the pure FE phase associated with uniaxial compression through Poisson's ratio. 
Somewhere above $200 \mathrm{MPa}$, however, the volumetric strain deviates markedly from the expected elastic response. This is a substantially higher stress than the $50 \mathrm{MPa}$ at which Fritz ${ }^{6}$ reported departure of the observed volume strain from the expected elastic response. As noted above, this is not simply a transition from linear to nonlinear elastic behavior; the volume strains become permanent above this stress level. Again, this departure from linearity cannot be explained by domain reorientation (though some is almost certainly occurring), because that is an isovolumetric process. ${ }^{6}$

Nor can this departure from linearity reasonably be attributed to some sort of pore collapse. Unlike our earlier investigations, ${ }^{7,8}$ we have not conducted scanning electron microscopy to directly rule out pore collapse as a factor in the experiments that we discuss here. This is because the test specimens were completely destroyed in testing. (Even though two experiments were halted prior to failure, the specimens were subsequently retested at elevated confining pressures for other purposes, and destroyed..$^{20}$ ) Stable pore collapse, accompanied by compressive volumetric strains, is often observed during hydrostatic and triaxial compression of, for instance, porous rock. ${ }^{21}$ Here, however, we are uniaxially compressing a fairly strong, obviously brittle ceramic, and the catastrophic failure of our test specimens confirms that we are in the brittle deformation regime. Under this condition, it is far more likely that the pores will act as stress concentrators, not collapsing but rather promoting axial crack nucleation. This in turn would result in anomalously large, tensile, lateral strains and rapid volume increases preceding or coincident with failure, the dilatant behavior that typifies brittle, pressure-sensitive materials, of which ceramics are a sub-class. ${ }^{21}$ The lower compressive strength of the more porous 541-1 ceramic is probably a manifestation of this phenomenon. Thus, we tentatively reject pore collapse as an explanation for any of the peculiar compressive strain phenomena that we discuss in this paper.

We are therefore left with onset of the $\mathrm{F}_{R 1} \rightarrow \mathrm{A}_{O}$ transformation as the remaining explanation for this large departure from linearity. In the example that we show for hifire 424 (Figure 4.1 ), this is at $\sigma_{1} \approx 260 \mathrm{MPa}$; for hifire $541-1$ (Figure 4.2 ), this is at $\sigma_{1} \approx 240 \mathrm{MPa}$. These values are very close to the values that we have determined for the pressures for transformation in hydrostatic compression. These results are not atypical.

In Figures 4.10 and 4.11 we summarize the volume strain data for our hydrostatic and uniaxial compression experiments on specimens from the two hifires. On these figures, we also plot the expected elastic volumetric response and the hydrostatic pressures for transformation (horizontal lines). We think it is clear from the data that, barring the early toes evident in many of the tests, volumetric strain response for the uniaxial compression 
experiments begins to deviate significantly from linearity at a value of $\sigma_{1}$ that is identical to the pressure for transformation under hydrostatic loading. This is consistent with the prediction that we made earlier in this paper, though different from Fritz's ${ }^{6}$ earlier result.

The unusually large amounts of compressive volume strain that the PNZT sustains prior to failure, $0.8-1.05 \%$ (Tables 4.1 and 4.2 ), further confirm the occurrence of the transformation in our experiment, and suggest to us that the reaction probably continues right up to failure. Fritz ${ }^{6}$ reached the same conclusion. Cao and Evans ${ }^{23}$ tested four different formulations of modified lead zirconate titanate in uniaxial compression, including piezoelectric, ferroelectric relaxor and antiferroelectric compositions. They did observe large, nonlinear axial strains at failure in some instances (approximately $0.8 \%$ at $900 \mathrm{MPa}$ for a tin+lanthanum-doped antiferroelectric), presumably caused by domain switching. However, they report that in all cases, volumetric strains were linear and nonhysteretic (i.e., elastic) unless microcracking occurred. And when microcracking did occur, volume strain was predictably dilational, as discussed above, not compressive. Linear extrapolation of their volume strain data for the antiferroelectric formulation suggests a compressive volume strain of at most $0.35 \%$ at failure at about $900 \mathrm{MPa}$. The volume strain at failure (approximately $500 \mathrm{MPa}$ ) for a relaxor ferroelectric ceramic was less than $0.1 \%{ }^{23, F i g .12 b}$ Volume strains at compressive failure for brittle, crystalline rock at room temperature are similarly low in uniaxial (and even triaxial) compression. ${ }^{22, F i g .31}$. The compressive volume strains that we have observed in uniaxial compression on PNZT are up to 3 times greater than the largest volume strains reported by Cao and Evans, ${ }^{23}$ and an order of magnitude greater than the smallest. This, we believe, is further convincing evidence that the FE-AFE transformation is occurring in our experiments, and continues up until failure. As discussed below, capacitance data are also consistent with these conclusions.

Beyond $\epsilon_{v} \approx 0.6 \%$, the ceramics begin to stiffen (Figures 4.1, 4.2, 4.10 and 4.11), although the net strains remain compressive right up until catastrophic failure. The apparent stiffening of the $\sigma_{1}$-volume strain plots in the late stages of deformation could be attributed to either (1) near-complete conversion of the FE phase to the AFE phase with accompanying changes in elastic properties, (2) the onset of microcracking, which would have the same apparent effect, or (3) some combination of the two phenomena. ${ }^{22,23}$ It is interesting to observe that the lateral strains begin to reverse again at approximately at this time (Figures 4.1 and 4.2), also consistent with either phenomenon, or some combination of the two.

A few additional remarks about the nonlinear axial and lateral strain behavior are in order before moving on to discussion of the capacitance mea- 
Capacitance $(\mathrm{pF})$

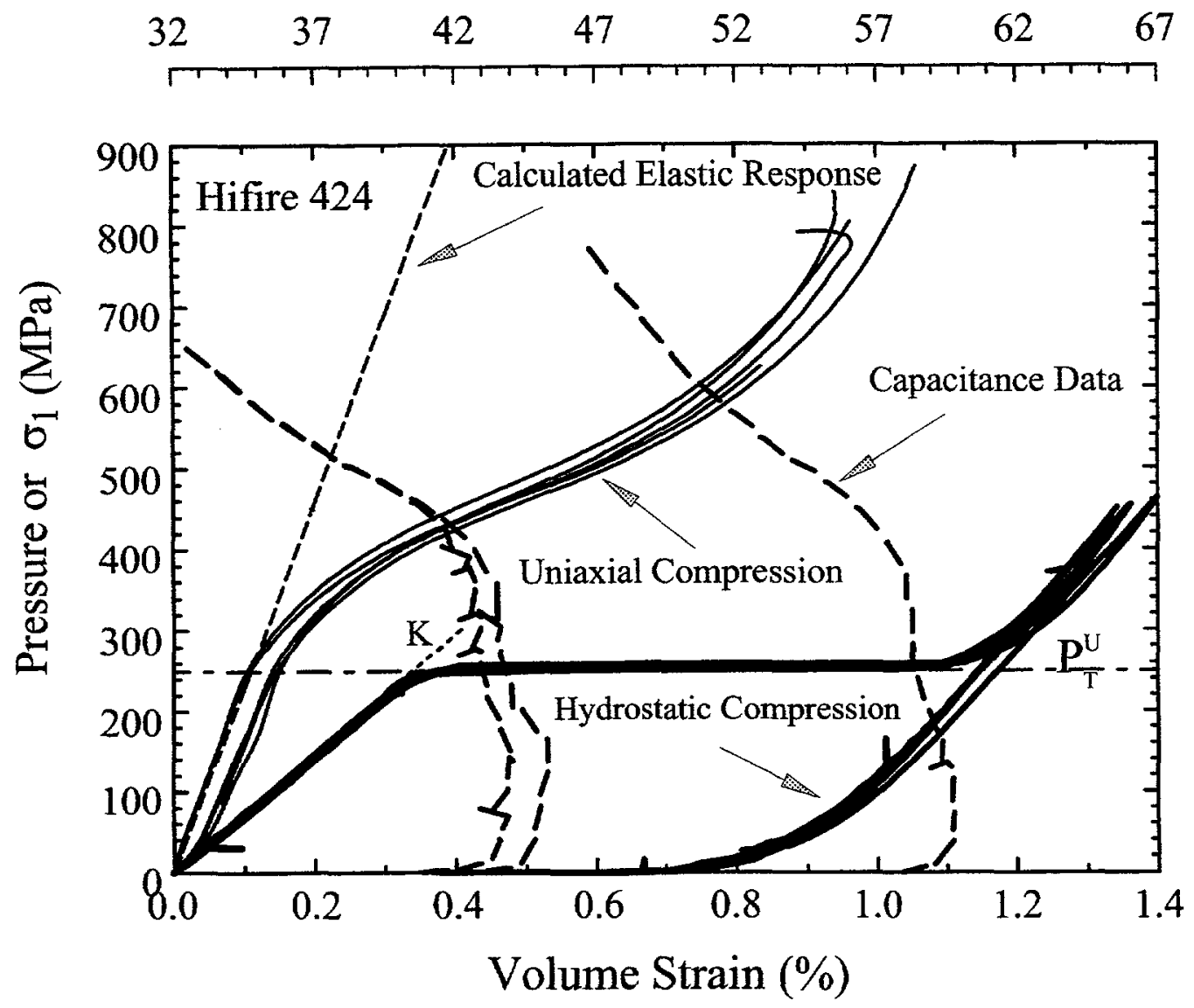

Figure 4.10: Summary plot comparing volume strain behavior of five uniaxial compression experiments on unpoled 424 ceramic with three hydrostatic compression experiments on the same material. ${ }^{7,8}$ Capacitance data for three uniaxial compression experiments are also shown. Disregarding the early nonlinear behavior below 40 $\mathrm{MPa}$, it is clear that the transformation begins in uniaxial compression when $\sigma_{1}=P_{T}^{U}$. Calculated elastic volumetric response in uniaxial compression is shown, as is the bulk modulus calculated using $\mathbf{E}, \nu$, and the assumption of mechanical isotropy (line labeled "K"). 
Capacitance $(\mathrm{pF})$

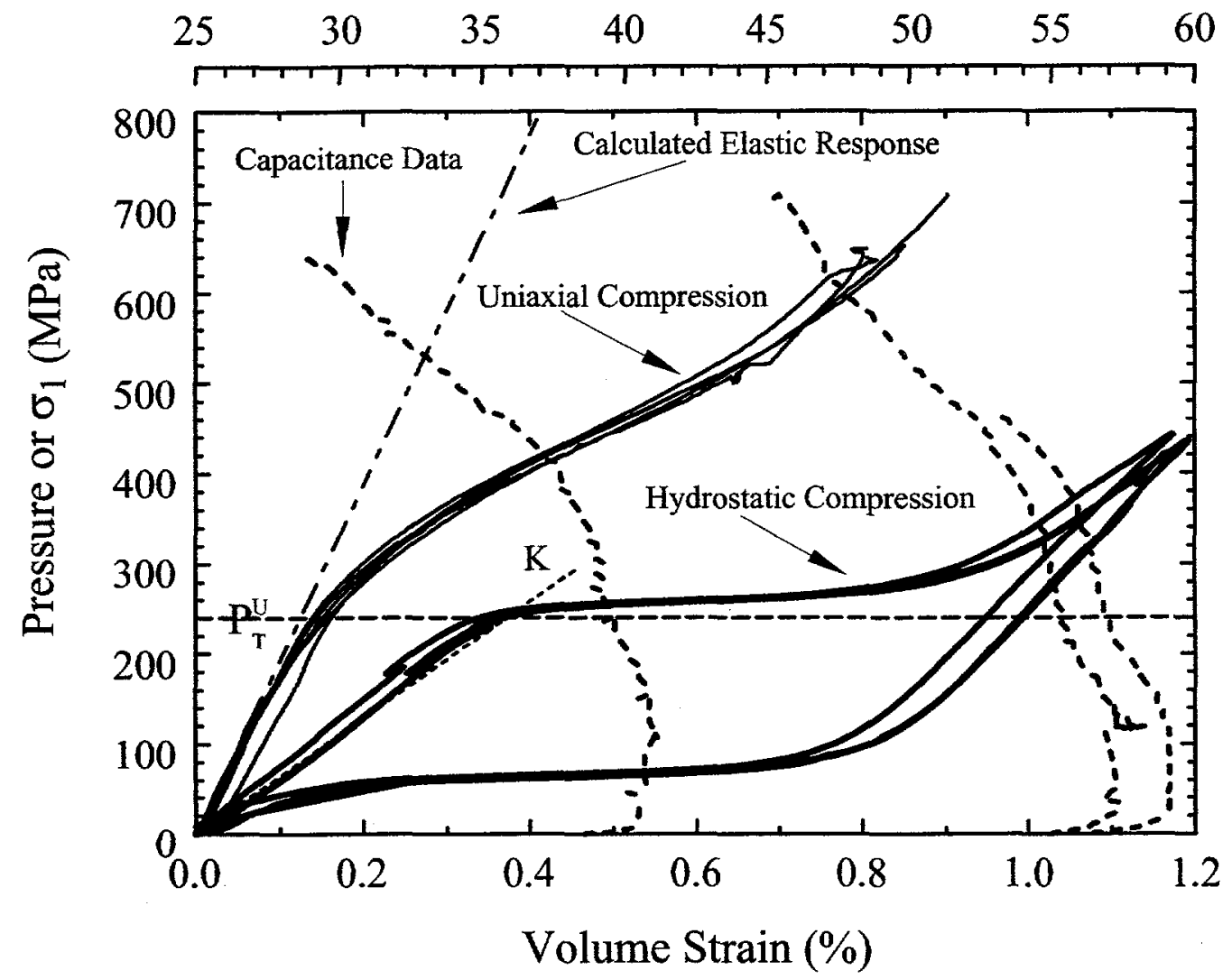

Figure 4.11: Summary plot comparing volume strain behavior of five uniaxial compression experiments on unpoled 541-1 ceramic with three hydrostatic compression experiments on the same material. ${ }^{7,8}$ Capacitance data for three uniaxial compression experiments are also shown. Disregarding the early nonlinear behavior below $40 \mathrm{MPa}$, it is clear that the transformation begins in uniaxial compression when $\sigma_{1}=P_{T}^{U}$. Calculated elastic volumetric response in uniaxial compression is shown, as is the bulk modulus calculated using $\mathbf{E}, \nu$, and the assumption of mechanical isotropy (line labeled "K"). 
surements. Upon axial loading, the axial strain gauges record compression, and the lateral gauges record rarefaction, as would be expected; the strains appear to be approximately linear up to about $100 \mathrm{MPa}$ (Figures 4.1 and 4.2). With increased loading, however, both the lateral (tensile) and axial (compressive) strains suddenly increase with respect to $\sigma_{1}$ around $100 \mathrm{MPa}$, while the volume strain remains linear. Domain realignment in response to the stress almost certainly explains this anomalous behavior. ${ }^{6}$ This is because the unit cell is elongated in the polar direction, which tends to switch away from the applied compressive stress. ${ }^{6,18}$ Disproportionately large axial compressive and lateral tensile strains would result.

With increased loading, the lateral strains undergo their first complete reversal at about $360-380 \mathrm{MPa}$ actually recording contraction for an extended period (Figures 4.1, 4.2, 4.10 and 4.11). Domain realignment cannot explain lateral contractions during compression. Thus, like Fritz ${ }^{6}$ before us, we are forced to conclude that the $F_{R 1} \rightarrow A_{O}$ transformation is the only likely explanation for the phenomenon, reinforcing our earlier observations and conclusions. The volume reduction caused by the transformation overwhelms the tensile strains caused by elastic response and domain switching effects. Of course, the transformation must begin at a stress well below the point of complete reversal of the lateral strains, but it is probably impossible to identify that stress from the lateral strains alone. Although the elastic contribution to the lateral strains can be accurately estimated and subtracted out, it is more difficult to identify the contribution from domain switching, and the range of stresses over which it operates.

With continued axial compression, the lateral strains eventually reverse once again. Failure consistently closely follows the onset of this second reversal.

In summary, strain measurements from uniaxial compression experiments suggest the following sequence of events. Domain switching begins to occur in earnest at a stress of approximately $100 \mathrm{MPa}$. However, occasional nonlinearity in the axial and lateral strain records indicates that some domain switching may occur even at the lowest stresses in our experiments. Volumetric strain response is essentially linear up to $P_{T}^{U}$ (about $250 \mathrm{MPa}$ ), and consistent with elastic behavior. When $\sigma_{1}=P_{T}^{U}$, the $F_{R 1} \rightarrow A_{O}$ transformation begins. This is a substantially higher stress than that reported by Fritz, ${ }^{6} 50 \mathrm{MPa}$. The reason for this discrepancy is not known. We suggest that the "toe" behavior that we observe at low stresses may be the result of transient stress concentrations caused by slight loading eccentricities, which might trigger the transformation locally; the reaction might terminate when the concentration is relieved. If Fritz' $\mathrm{s}^{6}$ experiments were similarly affected, this might resolve the discrepancy. 


\subsection{Capacitance Measurements and Onset of the Transformation}

Dielectric behavior has proven to be an important diagnostic for the weak, first-order FE-AFE transformation in PNZT in both hydrostatic compression, ${ }^{3,6,7}$ and in our CSD experiments. ${ }^{6,7}$ In an effort to confirm the stress at which the transformation began, we monitored the capacitance of three test specimens from each hifire during uniaxial compression (Tables 4.1 and 4.2). Capacitance data from two typical uniaxial compression experiments are plotted on the right-hand ordinate against volume strain in Figures 4.I and 4.2. Capacitance data for all uniaxial compression experiments in which they were collected are shown in Figures 4.10 and 4.11. In these latter figures, pressure and $\sigma_{1}$ are plotted directly against capacitance on the upper abscissa.

Comparison with Figures 2.2 and 2.4 emphasizes the differences between the capacitance signatures of hydrostatic compression and CSD experiments on the one hand, and uniaxial compression experiments, on the other. In hydrostatic compression and CSD experiments, capacitance of 424 ceramic typically falls abruptly by $25-30 \mathrm{pF}$ at the phase transformation (Figure 2.2), and that of 541-1 ceramic typically rises by $10-15 \mathrm{pF}$ at the transformation, subsequently falling again (Figure 2.4). As noted in our earlier investigations, the reason for the difference between hifires is unknown.

In contrast, the capacitance signatures that we observe for hifire 424 and 541-1 ceramic are quite similar in uniaxial compression, yet very different from their counterparts observed in hydrostatic or CSD experiments. The signature is consistently characterized (Figures $4.1,4.2,4.10$ and 4.11) by a small rise (2-3 $\mathrm{pF})$ in capacitance upon loading, plateauing at about 20-30 $\mathrm{MPa}$. Capacitance begins to drop once more (again by only $2-3 \mathrm{pF}$ ) at about $100-110 \mathrm{MPa}$, again plateauing (hifire 424) or going through an inflection point (hifire 541-1) at 250-260 MPa, the uniaxial stress that matches the transformation pressure under hydrostatic conditions. At about 350 (hifire 541-1) to $400 \mathrm{MPa}$ (hifire 424) the capacitance begins to drop more rapidly and continuously (by about 10-12 pF), until failure. Overall changes in capacitance (10-13 pF: Figures 4.1 and 4.2) are somewhat lower than in our earlier experiments (up to $30 \mathrm{pF}$ : Figure 2.2), and there are no obvious, abrupt changes in capacitance at the axial stress at which the volume strain data suggest the phase transformation starts in earnest (Figures 4.1, 4.2, 4.10 and 4.11).

Despite the seemingly great differences from earlier capacitance signatures, we can be certain that the results from the uniaxial compression experiments can be attributed to occurrence of the phase transformation. Though 
the overall changes in capacitance of the uniaxial compression experiments are smaller than those for the hydrostatic and CSD experiments, they are, nevertheless, too large to be attributed to strain effects alone. Straightforward calculations using Eq. (4.1), below, and assuming that the relative dielectric constant for PNZT is about $250,{ }^{3}$ show that the effects of the small strains that we observe on capacitance should be in the range of $1-2 \%$. We can carry these calculations a step further and confirm that under our extreme loading conditions, changing sample dimensions are not in some way masking or offsetting real changes in dielectric properties, resulting in the peculiar capacitance measurements. Note that maximum resolved shear stresses at the transformation in these experiments-approximately $125 \mathrm{MPa}$-are almost twice the maximum applied in our earlier investigation-75 $\mathrm{MPa} .^{7,8}$

We can convert the capacitance measurement, which depends upon the specimen's electrode area and width of separation, to the relative dielectric constant, $\epsilon$, which is an intrinsic material property. We use the following relationship:

$$
\epsilon^{\prime}=\frac{C w}{\epsilon_{0} A_{e}}
$$

Here, $C$ is the measured capacitance, $w$ is the distance separating the specimen electrodes, $\epsilon_{0}$ is the permittivity of vacuum $\left(8.854 \mathrm{pF} \mathrm{m}^{-1}\right)$, and $A_{e}$ is the area of the electrodes. Because $A_{e}=l \times w$, where $l$ is the specimen length and the lateral strains are isotropic, the change in $\epsilon l$ is a function only of the measured capacitance and $l$, which is corrected for continuously using the measured axial strain. Results are plotted in Figures 4.12 and 4.13, and are qualitatively identical to the capacitance plots in Figures 4.1 and 4.2. Thus, strain effects do not appear to be masking some larger change in dielectric behavior.

The capacitance and dielectric changes therefore appear to be real, and too large to attribute to strain effects alone. We conclude that the overall changes confirm the occurrence of the phase transformation in our uniaxial compression experiments. Furthermore, the capacitance continues to decrease throughout the course of the experiments, never leveling out again as in our earlier $\mathrm{CSD}^{7,8}$ and hydrostatic compression tests (e.g., Figure 2.2). This indicates to us that the transformation is not entirely complete at failure.

But, for reasons that are not understood, the capacitance signatures do not give us the clear indicator of onset of the transformation that they provide under hydrostatic and CSD conditions, at least for hifire $424 .^{7-9}$ We think it is significant that the $\sigma_{1}$-capacitance plots for both hifires go through inflection points from concave upward to concave downward at the same stresses at 


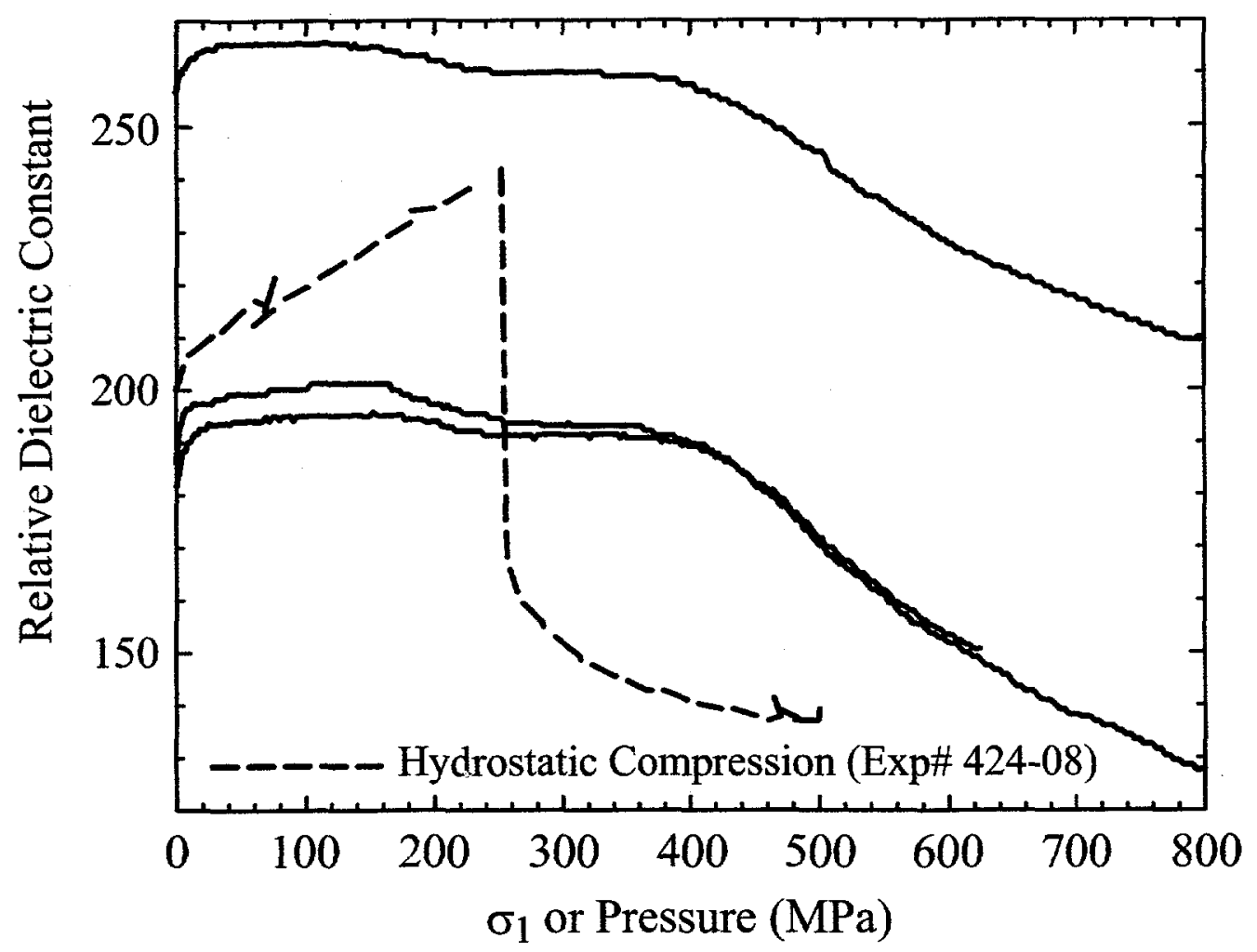

Figure 4.12: Relative dielectric constant measurements for uniaxial compression experiments on 424 ceramic. Similarity with capacitance data (Figure 4.10) confirm that specimen strains are not somehow "masking" expected behavior at the transformation. Results from a recent hydrostatic compression experiment are shown for comparison.

which strain data indicate that the transformation begins. Furthermore, beyond the inflection points, the capacitance continuously drops with increasing stress, just as we would expect if, as we argue, the $F_{R 1} \rightarrow A_{O}$ transformation is progressing above the critical stress. Thus, we conclude that the inflection point in the $\sigma_{1}$-capacitance record really does mark onset of the transformation. But we can offer no detailed explanation for the peculiar shape of the curve. In particular, why an inflection point instead of a steady drop in capacitance? We can only speculate that in uniaxial compression, several 


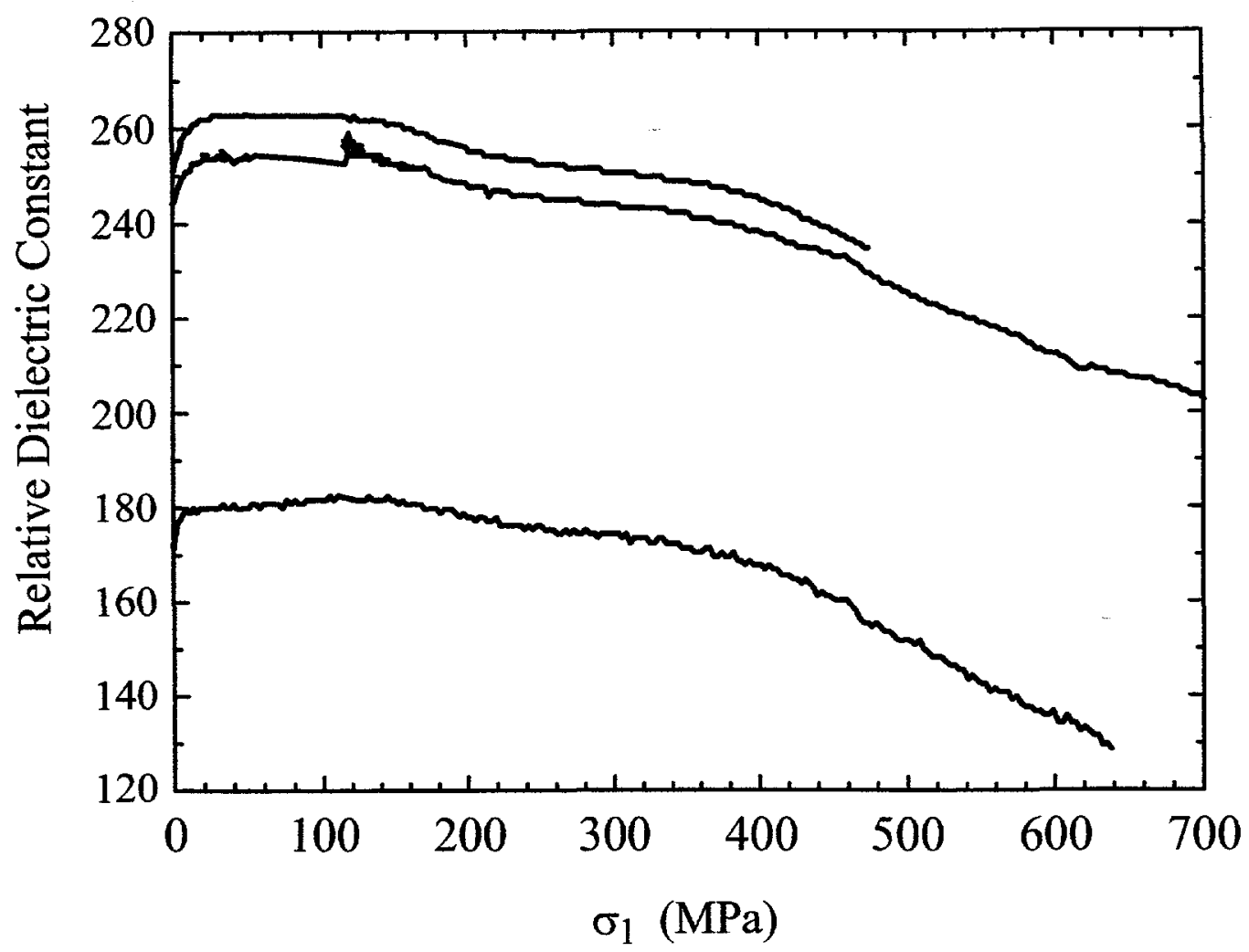

Figure 4.13: Relative dielectric constant measurements for uniaxial compression experiments on 541-1 ceramic. Similarity with capacitance data (Figure 4.11) confirm that specimen strains are not somehow "masking" expected behavior at the transformation.

processes that are absent or greatly reduced in hydrostatic compression and CSD experiments somehow combine to give this peculiar result. These include (1) dipole rotation, (2) early, localized $\mathrm{FE} \rightarrow \mathrm{AFE}$ transformation caused by transient stress concentrations, and (3) complex behavior of the lateral strains. The second process has not been confirmed, but could explain the early toe that we often observe, and the third should, in principle, only be a very small effect. 


\subsection{Summary of Results from Constant Strain Rate Experiments}

Volume strain measurements for constant strain rate experiments on 424 and 541-1 ceramic indicate that the $F_{R 1} \rightarrow A_{O}$ transformation begins when the uniaxial compressive stress equals the hydrostatic pressure at which the transformation would otherwise occur.

Capacitance data for these same experiments are more equivocal on this point. The total change in capacitance confirms that the transformation does indeed occur. The continuing decrease in capacitance further indicates that the transformation continues to occur up until failure. However, the capacitance signatures for the uniaxial compression experiments are otherwise very different from those observed in our earlier hydrostatic compression and CSD experiments. Following an initial rise, plateau and drop upon loading, the capacitance data merely go through an inflection point at the stress which coincides with the onset of the transformation as indicated by the volume strain data. Capacitance then continues to decrease, albeit at at different rate. This behavior is very different from what we observe in hydrostatic compression and CSD experiments on the two hifires.

Nevertheless, it is very repeatable, and we conclude that this consistent inflection point is confirmation that the transformation is indeed starting when the uniaxial compressive stress equals the pressure at which the transformation would begin under hydrostatic conditions. We do not understand the reason for the differences between the capacitance signatures for uniaxial compression experiments on the one hand, and hydrostatic compression and CSD experiments on the other. We suggest that it may be the result of competing processes that occur in uniaxial compression experiments which somehow offset or smear out the capacitance drop caused by the transformation.

The observed onset of the transformation when $\sigma_{1}=P_{T}^{U}$ agrees with our earlier prediction for uniaxial compression experiments. We also predicted that continually increasing stress should be required to drive the transformation to completion, and that when the load is held constant, the transformation should stop. We discuss the results of three such experiments in the following section. 


\subsection{Interrupted Uniaxial Compression Exper- iments}

Three uniaxial compression experiments were performed in which the displacement was held at three different points above the transformation stress, $\sigma_{1} \approx 250 \mathrm{MPa}$ (Figures 4.14-4.16). In the first experiment (Figure 4.14), displacement was held for periods of no more than $200 \mathrm{~s}$. In the second (Figure 4.15), displacement was held longer, for periods of $300-500 \mathrm{~s}$. In the third (Figure 4.16), displacement was held for periods of almost $1000 \mathrm{~s}$ each. Linear fits were performed to the data for each of the loading and holding intervals to quantify the changes in volumetric strain rate. Results were qualitatively the same for all three experiments.

During all of the holding stages, stresses dropped somewhat. Also, during all but one of the holding stages (Figure 4.16), volumetric strain increased slightly, indicating that the transformation did not altogether stop. However, the stress decreases seldom constituted more than $1-3 \%$ of the total axial stress, while the decreases in volumetric strain rate ranged from 1-3 orders of magnitude. Typically, the longer the duration of the holding period, the greater was the difference between volumetric strain rates of the deforming and holding stages.

In the first experiment (Figure 4.14), volumetric strain rates decreased by approximately 1-3 orders of magnitude during the holding stages, with a single order of magnitude decrease being most common. In the last experiment (Figure 4.16), decreases were uniformly greater than two orders of magnitude. In fact, in the first holding stage of this experiment, during which the the stress dropped by $6.5 \%$ (the greatest fractional decrease that we observed), volumetric strain was actually recovered, either as a result of elastic behavior, partial reversal of the transformation, or some combination thereof. The transition certainly was not proceeding. We take the steady decline in transformation rate with holding time as an indication that the transformation is slowing rapidly, despite some slight time dependence immediately upon holding the displacement.

In each instance, when loading was resumed the volumetric strain rate returned to approximately the value that it had prior to the holding period. These results confirm our second prediction, that the transformation requires a continually increasing stress to drive the reaction to completion in uniaxial compression. 


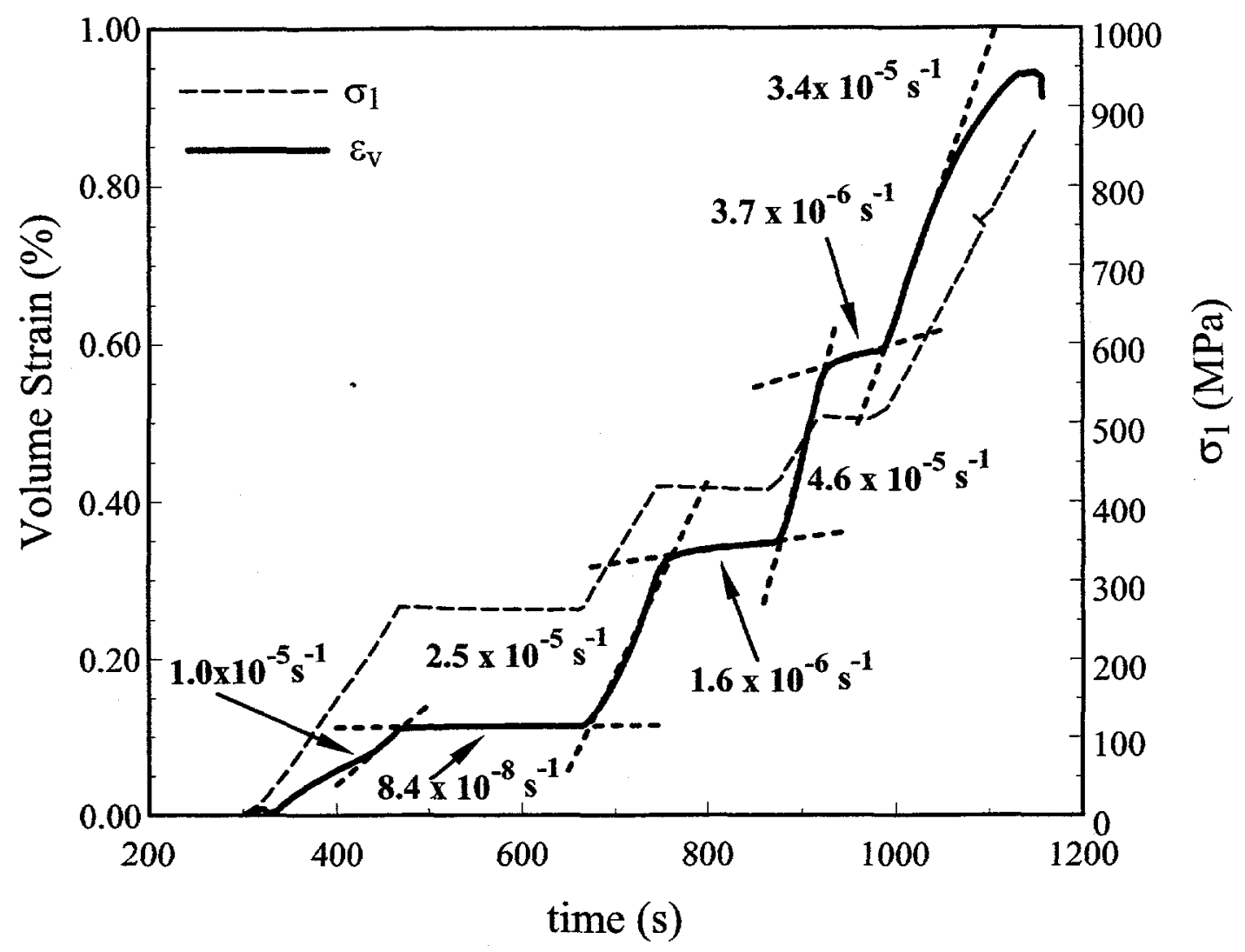

Figure 4.14: Plot of volume strain (bold, solid line) and $\sigma_{1}$ (fine, dashed line) versus time for unconfined, constant strain rate experiment on 424 ceramic in which displacement was halted three times. Coarse, dashed lines indicate volumetric strains rates determined from linear least-squares fits to the corresponding loading and holding stages. Note the 1 to 3 order of magnitude drops in volumetric strain rate when displacement is held. 


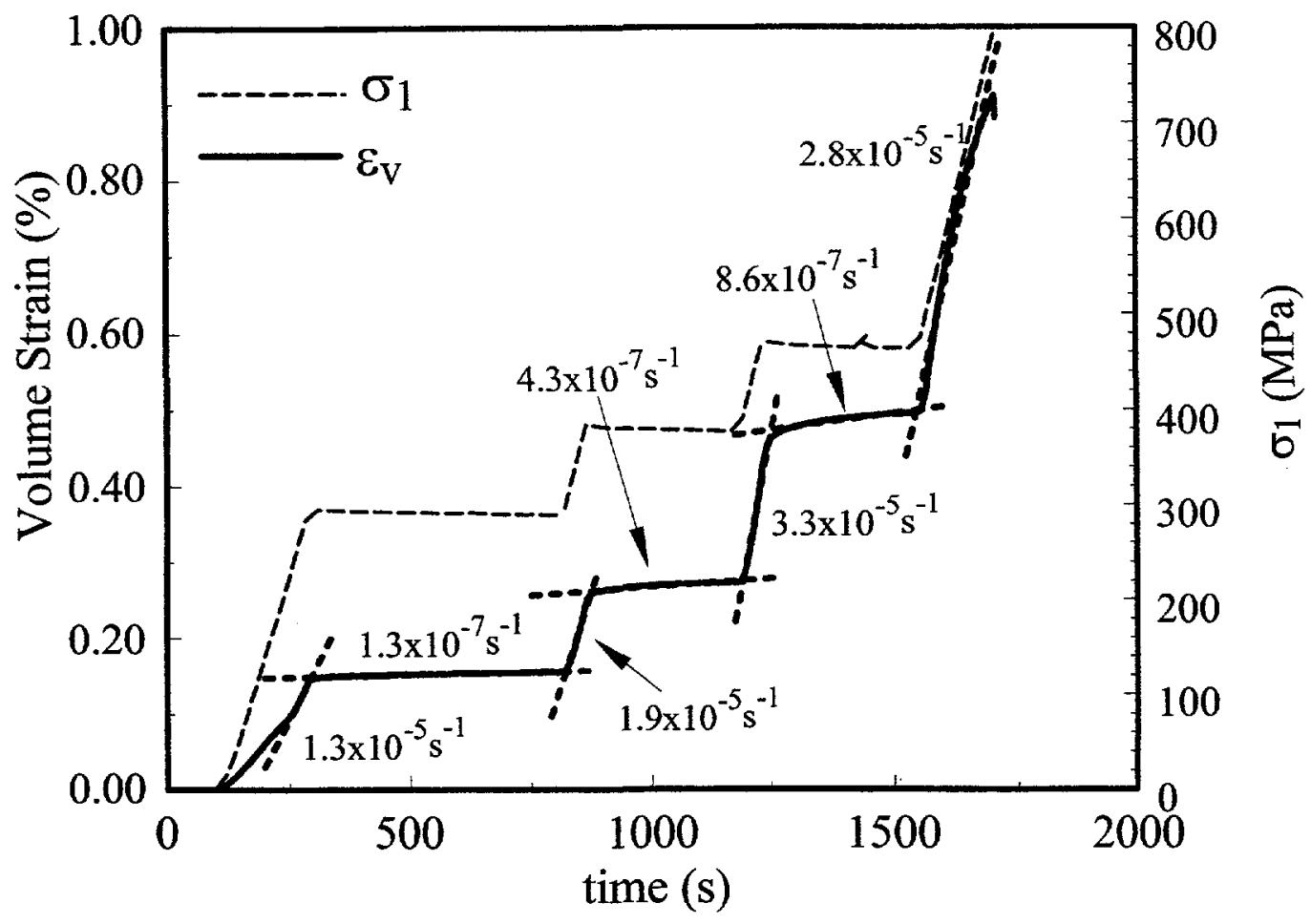

Figure 4.15: Plot of volume strain (bold, solid line) and $\sigma_{1}$ (fine, dashed line) versus time for unconfined, constant strain rate experiment on 424 ceramic in which displacement was halted three times. Coarse, dashed lines indicate volumetric strains rates determined from linear least-squares fits to the corresponding loading and holding stages. Note the 1 to 2 order of magnitude drops in volumetric strain rate when displacement is held. 


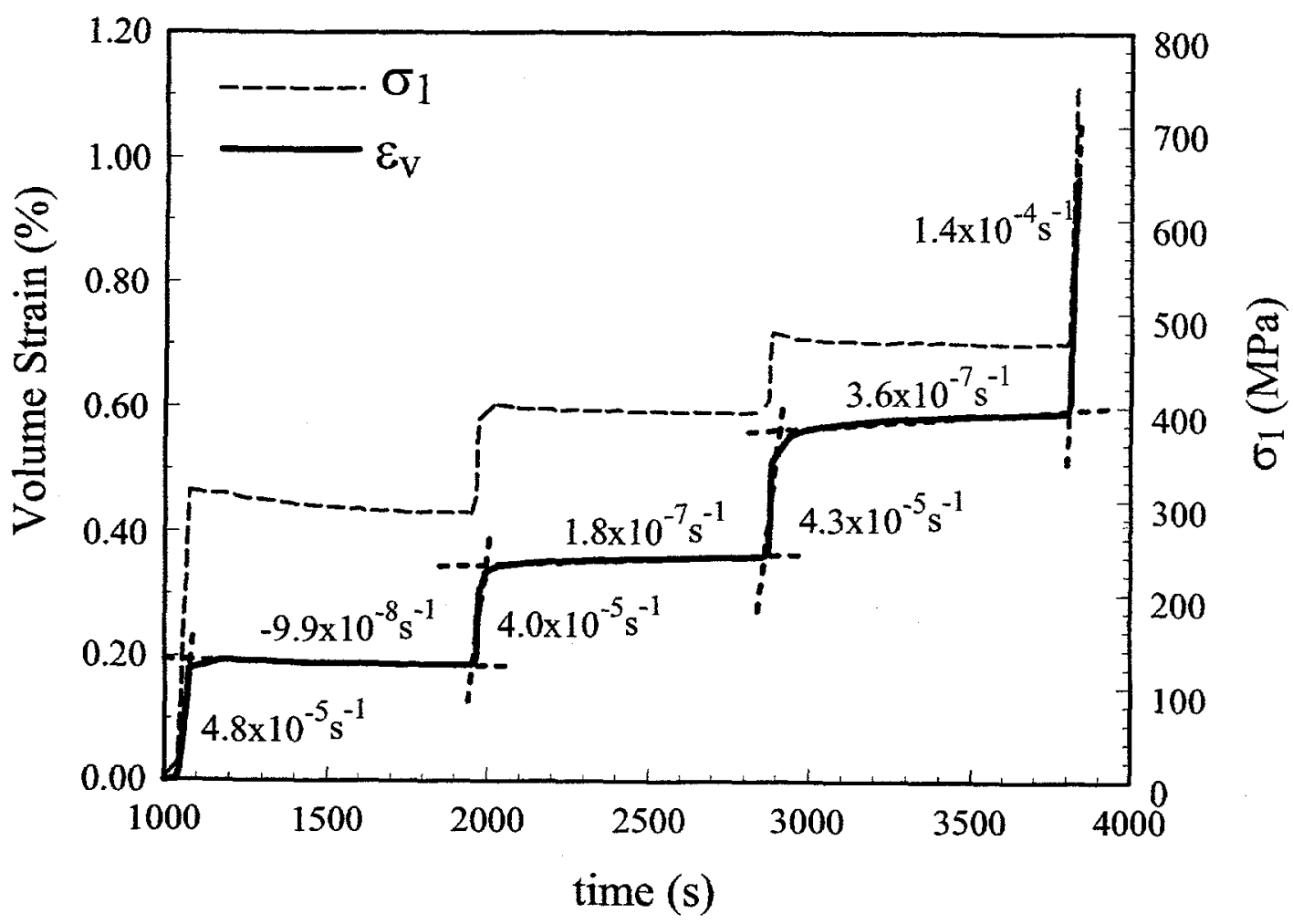

Figure 4.16: Plot of volume strain (bold, solid line) and $\sigma_{1}$ (fine, dashed line) versus time for unconfined, constant strain rate experiment on 424 ceramic in which displacement was halted three times. Coarse, dashed lines indicate volumetric strains rates determined from linear least-squares fits to the corresponding loading and holding stages. Note the two order-of-magnitude (or more) drops in volumetric strain rate when displacement is held. 


\section{Chapter 5}

\section{Discussion and Conclusions}

Based on evidence from hydrostatic and triaxial compression experiments on unpoled PNZT ceramic, we proposed that under nonhydrostatic loading, the $F_{R 1} \rightarrow A_{O}$ transformation begins when the maximum compressive stress equals the hydrostatic pressure at which the transformation would normally occur. ${ }^{9}$ Subsequent experiments on poled PNZT ceramic were inconsistent with this hypothesis ${ }^{10,11}$ Based on evidence from existing experiments on unpoled and poled ceramic, we have suggested here that the orientation of the stress relative to some feature of PNZT's structure, as well as the magnitude of the compressive stress may be critical for onset of the transformation. We have shown, for example, that for constant-stress-difference experiments on poled PNZT ceramic compressed normal to the poling direction, the transformation occurs when the compressive stress on a plane at $45^{\circ}$ to the polar axis equals the hydrostatic pressure for transformation. The reason for this is not clear, but confirms the importance of the orientation of the stress relative to the PNZT crystal structure. A correlary to this modified hypothesis is that the transformation should be complete when the mean stress equals the hydrostatic pressure for transformation.

Assuming that this modified hypothesis is true, we can quantitatively explain most of the effects of nonhydrostatic compression on the $F_{R 1} \rightarrow A_{O}$ transformation in unpoled PNZT which we have previously reported. Specifically, we have developed simple arithmetic expressions that accurately describe the effects of nonhydrostatic compression on the mean stress and confining pressure for transformation of unpoled ceramic. We can also develop a quantitative explanation for the apparent effects of nonhydrostatic compression on the transformation kinetics of unpoled ceramic at a fixed pressurization rate.

The model predicts several things about uniaxial compression experiments on unpoled, randomly oriented PNZT ceramic. First, it predicts that 
the transformation should begin when $\sigma_{1}=P_{T}^{U}$, and be complete when $\sigma_{m}=P_{T}^{U}$. Second, it predicts that a steadily increasing stress should be required to drive the transformation to completion. Results from our uniaxial compression experiments confirm both of these predictions, lending additional credence to our model.

Although we do not yet understand details of the relationship among the stress field, PNZT's crystal structure, and onset of the transformation, we nevertheless can add several important improvements to our numerical model for the transformation. We can now include a simple mathematical relationship that predicts onset of the transformation of unpoled ceramic when subjected to a general, nonhydrostatic, net-compressive state of stress. We now also have an empirical relationship linking transformation of poled ceramic to the resolved normal stress on a plane oriented at $45^{\circ}$ to both the poling direction and the maximum compressive stress. Insofar as we are aware at this time, the relationship only applies to the situation in which $\sigma_{1}$ is oriented perpendicular to the poling direction: so-called "normal-mode" compression. However, this is exactly the mode in which PNZT is used in the shock activated power supplies that we are currently modeling. We also have some quantitative insight into the relationship among transformation kinetics under hydrostatic and nonhydrostatic loading, rate of increase of pressure or mean stress, and charge release rates, at least for normal-mode compression.

Additional experimental work will be necessary to quantify and generalize the last two observations for poled ceramic. To address the first issue, we plan a series of CSD and uniaxial compression experiments in which the maximum compressive stress will be oriented at a range of angles to the poling direction. These experiments will be difficult to perform because we will need to develop a technique to pole all orientations to the same remanent polarization after machining in the unpoled state. We will address the second issue by a series of hydrostatic compression and CSD experiments in which the pressurization rates differ from that which we are currently using.

Another logical step will be to explore the relationship between nonhydrostatic loading and temperature for onset of the transformation. As noted previously, Coe and Paterson, ${ }^{14}$ showed that nonhydrostatic loading affected the temperature for onset of the displacive $\alpha \rightarrow \beta$ quartz transformation. Using single-crystals oriented at two different angles to the maximum compressive stress, they also demonstrated that the transformation temperature varied with orientation and magnitude of the compressive stress. They did not, however, generalize their result to polycrystals, or explore the relationships between transformation and stress state at constant temperature, which would have made their study more complete. 


\section{Chapter 6}

\section{References}

${ }^{1}$ P. C. Lysne and C. M. Percival, "Electric energy generation by shock compression of ferroelectric ceramics: normal-mode response of PZT 95/5," J. Appl. Phys, 46, 1519-1525 (1975).

${ }^{2}$ F. Bauer, K. Vollrath, Y. Fetiveau and L. Eyraud, "Ferroelectric ceramics: application to mechanical energy conversion under shock compression," Ferroelectrics, 10, 61-64 (1976).

${ }^{3}$ I. J. Fritz and J. D. Keck, "Pressure-temperature phase diagrams for several modified lead zirconate ceramics," J. Phys. Chem. Solids, 39, 11631167 (1978).

${ }^{4}$ D. Berlincourt, H. H. A. Krueger and B. Jaffe, "Stability of phases in modified lead zirconate with variation in pressure, electric field, temperature and composition," J. Phys. Chem. Solids, 25, 659-674, (1964).

${ }^{5}$ S. T. Montgomery, "Analysis of transitions between ferroelectric and antiferroelectric states under conditions of uniaxial strain," in: Y. M. Gupta (ed), "Shock Waves in Condensed Matter," pp.179-184, Pleunum Publishing Corporation, 1986.

${ }^{6}$ I. J. Fritz, "Stress effects in two modified lead zirconate titanate ferroelectric ceramics," J. Appl. Phys., 50, 5265-5271 (1979). 
${ }^{7}$ D. H. Zeuch, S. T. Montgomery, J. D. Keck and D. J. Zimmerer, "Hydrostatic and Triaxial Compression Experiments on Unpoled PZT 95/5$2 \mathrm{Nb}$ Ceramic: The Effects of Shear Stress on the $F_{R 1} \rightarrow A_{O}$ Polymorphic Phase Transformation," Rept. No. SAND92-0484, Sandia National Laboratories, Albuquerque, NM, 1992, 164pp. (Available from National Technical Information Service, U.S. Dept. of Commerce, 5285 Port Royal Rd., Springfield, VA 22161.)

${ }^{8}$ D. H. Zeuch, S. T. Montgomery and J. D. Keck, "Hydrostatic and triaxial compression experiments on unpoled PZT 95/5-2Nb ceramic: the effects of shear stress on the $F_{R 1} \rightarrow A_{O}$ polymorphic phase transformation," J. Mater. Res., 7, 3314-3332 (1992).

${ }^{9}$ D. H. Zeuch, S. T. Montgomery and J. D. Keck, "Further obervations on the effects of nonhydrostatic compression on the $F_{R 1} \rightarrow A_{O}$ polymorphic phase transformation in niobium-doped, lead-zirconate-titanate ceramic," J. Mater. Res., 9, 1322-1327 (1994).

${ }^{10}$ D. H. Zeuch, S. T. Montgomery and D. J. Zimmerer, "The Effects of Non-Hydrostatic Compression and Applied Electric Field on the Electromechanical Behavior of Poled PZT 95/5-2Nb Ceramic During the $F_{R 1} \rightarrow A_{O}$ Polymorphic Phase Transformation," Rept. No. SAND951951, Sandia National Laboratories, Albuquerque, NM, 1995, 114pp. (Available from National Technical Information Service, U.S. Dept. of Commerce, 5285 Port Royal Rd., Springfield, VA 22161.)

${ }^{11}$ D. H. Zeuch, S. T. Montgomery and D. J. Holcomb, "The effects of non-hydrostatic compression and applied electric field on the electromechanical behavior of poled PZT $95 / 5-2 \mathrm{Nb}$ ceramic during the $F_{R 1} \rightarrow A_{O}$ polymorphic transformation," submitted to J. Mater. Res., (1998).

${ }^{12}$ F. C. Larché, "Coherent phase transformations," Ann. Rev. Mater. Sci., 20, 83-99 (1990).

${ }^{13}$ I. Shimizu, "Nonhydrostatic and nonequilibrium thermodynamics of deformable materials," J. Geophys. Res., 97, 4587-4597 (1992). 
${ }^{14}$ Coe, R. S., and M. S. Paterson, "The $\alpha \rightarrow \beta$ inversion in quartz: a coherent phase transition under nonhydrostatic stress," J. Geophys. Res., 74, 4921-4948 (1969).

${ }^{15}$ D. H. Zeuch, S. T. Montgomery and D. J. Holcomb, "Further evidence for a 'maximum compressive stress' criterion for onset of the $F_{R 1} \rightarrow$ $A_{O}$ transformation in PZT $95 / 5-2 \mathrm{Nb}$ ceramic under nonhydrostatic loading," presented at The Pacific Coast Regional and Basic Science Division Meeting of the American Ceramic Society, 12-15 October 1997, San Francisco, CA.

${ }^{16}$ W. D. Means, "Stress and Strain: Basic Concepts of Continuum Mechanics for Geologists," Springer-Verlag, New York, 339pp., 1976.

${ }^{17}$ R. H. Dungan and L. J. Storz, "Relation between chemical, mechanical and electric properties of $\mathrm{Nb}_{2} \mathrm{O}_{5}$-modified $95 \mathrm{~mol} \% \mathrm{PbZrO}_{3}-5 \mathrm{~mol} \%$ $\mathrm{PbTiO}_{3}$," J. Am. Ceram. Soc., 68, 530-533 (1985).

${ }^{18}$ I. J. Fritz, "Uniaxial-stress effects in a 95/5 lead zirconate titanate ceramic," J. Appl. Phys., 49, 4922-4928 (1978).

${ }^{19}$ R. D. Hardy, "Event Triggered Data Acquisition in the Rock Mechanics Laboratory," Rept. No. SAND93-0256, Sandia National Laboratories, Albuquerque, NM, 120pp. (Available from National Technical Information Service, U.S. Dept. of Commerce, 5285 Port Royal Rd., Springfield, VA 22161.)

${ }^{20}$ D. H. Zeuch, unpublished work

${ }^{21}$ T.-f. Wong, H. Szeto and J. Zhang, "Effect of loading path and porosity on the failure mode of porous rocks,"in: V. C. Li (ed), "Micromechanical Modelling of Quasi-Brittle Materials Behavior," pp. 281-294, American Society of Mechanical Engineers, New York, 1992.

${ }^{22}$ M. S. Paterson, "Experimental Rock Deformation: The Brittle Field." Springer-Verlag, New York, 254pp, 1978.

${ }^{23}$ H. Cao and A. G. Evans, "Nonlinear deformation of ferroelectric ceramics," J. Am. Ceram. Soc., 76, 890-896 (1993). 


\section{Distribution}

\section{External Distribution}

F. W. Davies

Ktech Corp.

2201 Buena Vista, SE

Suite 400

Albuquerque, NM 87110

Prof. Y. Horie

Dept. of Civil Engineering

North Carolina State University

Raleigh, NC 27695

L. M. Lee

Ktech Corp.

2201 Buena Vista, SE

Suite 400

Albuquerque, NM 87110

Prof. J. J. Mecholsky, Jr.

Dept. of Materials Science and Engineering

256A Rhines Hall

University of Florida

Gainsville, FL 32611

Prof. D. A. Payne

Dept. of Materials Science

302 Ceramic Engineering

University of Illinois

105 S. Goodwin

Urbana, IL 61801

Prof. D. Viehland

Department of Materials Science 209 Ceramic Engineering

University of Illinois

105 S. Goodwin

Urbana, IL 61801

\section{Sandia Internal}

MS-0303 M. J. Forrestal, 2414

MS-0311 G. E. Clark, 2671

MS-0328 J. A. Wilder, 2674

MS-0367 L. W. Carlson, 1833

MS-0367 S. J. Glass, 1833

MS-0437 C. S. Lo, 9117

MS-0443 J. G. Arguello, 9117

NS-0443 S. N. Burchett, 9117

MS-0443 A. F. Fossum, 9117

MS-0479 G. A. Kinemond, 2151

MS-0515 F. M. Bacon, 1561

MS-0515 S. G. Barnhart, 1561

MS-0515 T. A. Haverlock, 1561

MS-0515 J. D. Keck, 1561

MS-0515 D. K. Morgan, 1561

MS-0516 G. L. Laughlin, 1564

MS-0521 T. J. Young, 1567

MS-0521 R. A. Damerow, 1567

MS-0521 L. D. Interrante, 1567

MS-0521 S. T. Montgomery, 1567 (15)

MS-0521 R. A. Pike, 1567

MS-0521 T. W. Scofield, 1567

MS-0603 I. J. Fritz, 1712

MS-0751 J. M. Grazier, 6117

MS-0751 D. J. Holcomb, 6117

MS-0751 D. H. Zeuch, 6117 (15)

MS-0751 File, 6117

MS-0819 J. S. Peery, 9231

MS-0819 E. A. Boucheron, 9231

MS-0819 T. G. Trucano, 9231

MS-0819 J. R. Weatherby, 9231

MS-0820 P. Yarrington, 9232

MS-0820 M. B. E. Boslough, 9232

MS-0820 R. M. Brannon, 9232

MS-0820 D. A. Crawford, 9232 
MS-0820 H. E. Fang, 9232

MS-0820 M. E. Kipp, 9232

MS-0834 J. B. Aidun, 9117

MS-0871 N. A. Lapetina, 14405

MS-0873 R. J. Stiers, 14302

MS-0953 W. E. Alzheimer, 1500

MS-0953 J. T. Cutchen, 1501

MS-0959 F. P. Gerstle, Jr., 1492

MS-0959 S. J. Lockwood, 1492

MS-0959 R. H. Moore, 1492

MS-0959 P. Yang, 1492

MS-0986 J. H. Perry, 2691

MS-1111 T. V. Russo, 1734

MS-1159 W. H. Barrett, 9344

MS-1168 M. D. Furnish, 9321

MS-1181 J. R. Asay, 9511

MS-1181 L. C. Chhabildas, 9511

MS-1186 R. J. Lawrence, 9574

MS-1223 P. J. Wilson, 5202

MS-1349 W. F. Hammetter, 1846

MS-1349 A. J. Hurd, 1841

MS-1405 B. A. Tuttle, 1812

MS-1405 J. A. Voigt, 1846

MS-1411 D. B. Dimos, 1831

MS-1411 V. Tikare, 1834

MS-1421 G. A. Samara, 1152

MS-1421 M. U. Anderson, 1152

MS-1421 R. E. Setchell, 1152

MS-1434 G. E. Pike, 1802

MS-9006 D. J. Bohrer, 2200

MS-0619 Review \& Approval Desk, 15102 for DOE/OSTI

MS-0899 Technical Library, 4916 (2)

MS-9018 Central Technical Files, 8940-2 